\title{
Improving spineboard design
}

Citation for published version (APA):

Hemmes, B. (2016). Improving spineboard design: evaluation of a new, soft-layered spineboard in trauma care. [Doctoral Thesis, Maastricht University]. Datawyse / Universitaire Pers Maastricht.

https://doi.org/10.26481/dis.20160121bh

Document status and date:

Published: 01/01/2016

DOI:

10.26481/dis.20160121bh

Document Version:

Publisher's PDF, also known as Version of record

\section{Please check the document version of this publication:}

- A submitted manuscript is the version of the article upon submission and before peer-review. There can be important differences between the submitted version and the official published version of record.

People interested in the research are advised to contact the author for the final version of the publication, or visit the DOI to the publisher's website.

- The final author version and the galley proof are versions of the publication after peer review.

- The final published version features the final layout of the paper including the volume, issue and page numbers.

Link to publication

\footnotetext{
General rights rights.

- You may freely distribute the URL identifying the publication in the public portal. please follow below link for the End User Agreement:

www.umlib.nl/taverne-license

Take down policy

If you believe that this document breaches copyright please contact us at:

repository@maastrichtuniversity.nl

providing details and we will investigate your claim.
}

Copyright and moral rights for the publications made accessible in the public portal are retained by the authors and/or other copyright owners and it is a condition of accessing publications that users recognise and abide by the legal requirements associated with these

- Users may download and print one copy of any publication from the public portal for the purpose of private study or research.

- You may not further distribute the material or use it for any profit-making activity or commercial gain

If the publication is distributed under the terms of Article $25 \mathrm{fa}$ of the Dutch Copyright Act, indicated by the "Taverne" license above, 


\section{Improving spineboard design}

evaluation of a new, soft-layered spineboard in trauma care

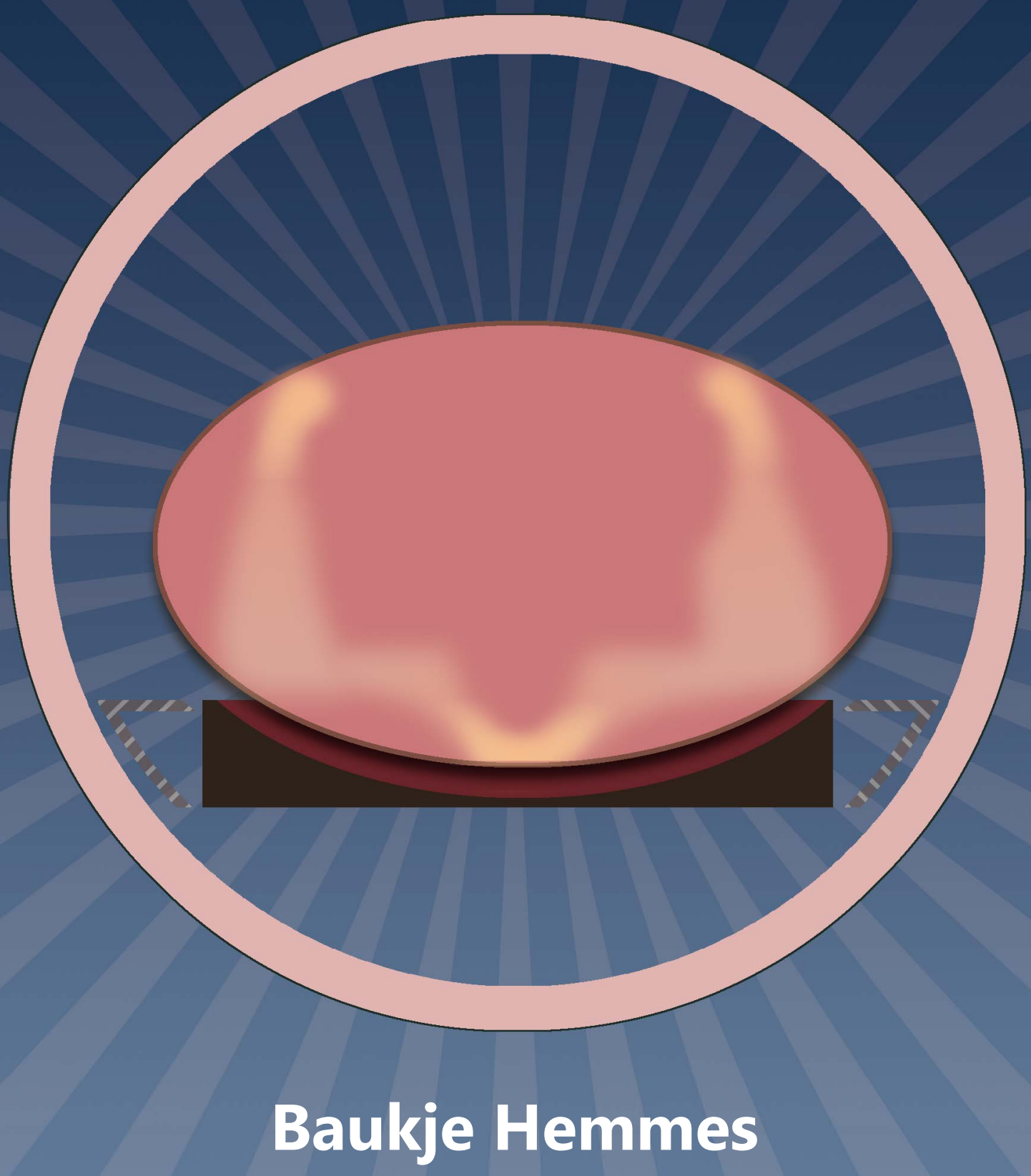





\title{
Improving spineboard design
}

\section{evaluation of a new, soft-layered spineboard in trauma care}

\author{
Proefschrift \\ ter verkrijging van de graad van doctor \\ aan de Universiteit Maastricht \\ op gezag van de Rector Magnificus Prof dr. L.L.G. Soete, \\ volgens het besluit van het College van Decanen, \\ in het openbaar te verdedigen \\ op donderdag 21 januari 2016 om 10:00 uur
}

door

Bauke Hemmes

geboren 21 januari 1976

te Hilversum

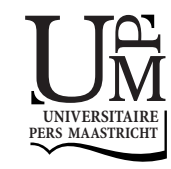


Promotor:

Prof. dr. P.R.G. Brink

Copromotor:

Dr. M. Poeze

Beoordelingscommissie:

Prof. dr. N.D. Bouvy (voorzitter)

Prof. dr. L.P.H. Leenen (Universiteit Utrecht)

Dr. K. Meijer

Dr. P.R. Worsley (Southampton University)

ISBN 9789461595140

Copyright B. Hemmes, Maastricht, The Netherlands, 2016

Cover design: Menke van Daalen \& Baukje Hemmes

Printed by Datawyse | Universitaire Pers Maastricht 


\section{Contents}

Chapter 1 Introduction

General background and outline of the thesis

Chapter 2 Reduced tissue-interface pressure and increased comfort on a newly developed soft-layered spineboard

Chapter 3 Effects of unconsciousness during spinal immobilisation on tissueinterface pressures

A randomised controlled trial comparing a standard rigid spineboard with a newly developed soft-layered long spineboard

Chapter 4 A numerical study of the risk for pressure ulcer development on a spineboard

Chapter 5 Cytokine IL-1 $\alpha$ as a marker of tissue damage during spineboard immobilisation. A prospective, randomised open-label crossover trial

Chapter 6 Effects of spineboard and headblocks on the image quality of head CT scans

Chapter 7 Effect of spinal immobilisation devices on radiation exposure in conventional radiography and computed tomography

Chapter 8 Summary and conclusions

Samenvatting en conclusies

Chapter 9 Valorisation

Dankwoord 



\section{Introduction}

General background and outline of the thesis
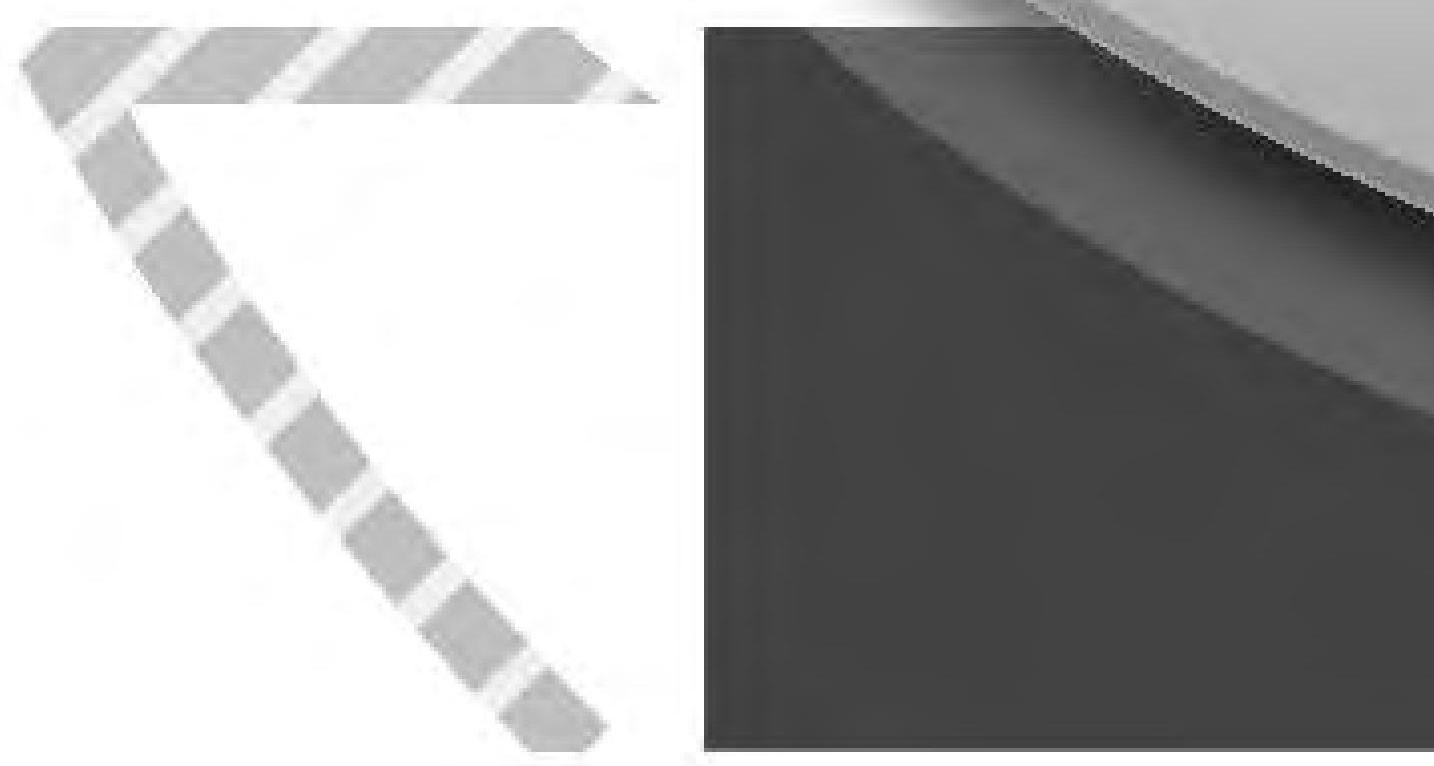

Baukje Hemmes 


\section{The origins of external immobilisation of the spine}

The first patent application for a device resembling the current spineboard was filed as early as 1936 [1] and was intended for "the safe carrying of injured persons", specifically for carrying trauma victims out of mines. Earlier devices for retrieval of injured miners existed, but were deemed unsuitable because they were made of quickly deteriorating canvas. Furthermore, these soft stretchers were not considered specifically suitable for handling patients with a spinal injury, nor were they practical in the transport of these patients, who were placed on a mine cart to get them out of the mine. Therefore, a device was developed with two specific features: it had a rigid surface, and it had a longitudinal slot along the board (Figure 1.1a). A rigid surface was chosen not only to facilitate easy transport of the patient out of the mines, but it had the added advantage of acting as a splint for broken limbs. The slot in the middle of the spineboard was designed to receive any protruding dislocated portion of the spinal column. The slot ensured that no pressure was exerted directly on the spinal column, thereby aiming to prevent secondary damage to the spine. Although not specifically designed with pain-relief in mind, allowing the spine to "float" over the slot also was considered to decrease pain by preventing pressure on the (possibly) injured body parts. Strikingly, spineboards currently in use no longer have this slot (Figure 1.1b).
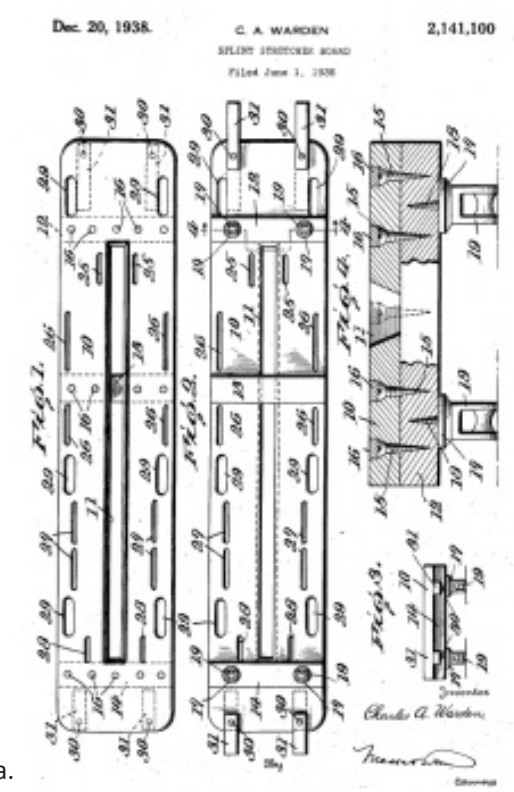

b.

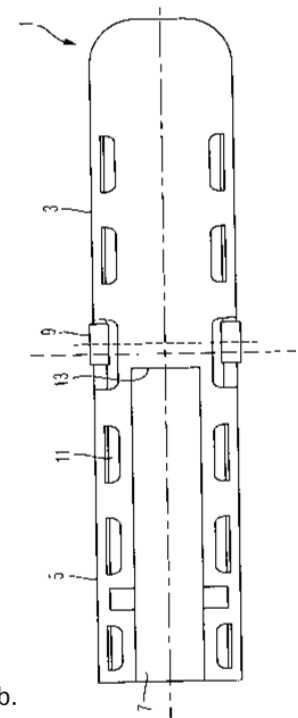

Figure 1.1a-b. Spineboard patents. a.patent by Warden (1936); b. patent by Mohr (2012) 
The use of a rigid spineboard to prevent secondary damage to an already injured spine may seem obvious nowadays, but it was a completely novel idea in 1936 . There was no scientific evidence showing that secondary damage to the spine could be prevented by using a rigid spineboard or another prehospital immobilisation device. Nevertheless, maintaining alignment and immobilisation of the spinal column were considered important to prevent secondary damage even in the early years of structured prehospital care [2-4]. Interest in experimental studies of the biomechanics of spinal damage only developed after the invention of the rigid spineboard. The earliest studies, performed in the mid-twentieth century, showed that direct vertical forces of 250-275 pounds (113-124 kg) were needed to produce the first signs of vertebral fractures [5], and a force of about 300 pounds (135 kg) was needed to irreversibly damage the intervertebral disc [6]. No data was generated about whether the spineboard could actually protect the spine from further damage during extrication and transport. Nevertheless, at the end of the 1960s studies were published which stated that the spineboard should be used to extricate and transport victims [7-9], especially those with spinal injuries, to prevent further damage to the spine. In the 1980s, the first patents for vacuum mattresses for use in prehospital care [10] were applied for, with the specific aim of having a mattress which adapted to the patient and their injuries. Despite the lack of evidence, the use of spineboards and vacuum mattresses has since then been included in guidelines for trauma care worldwide and has been part of the gold standard for trauma care ever since [11].

Over fifteen years ago, Hauswald et al [12] concluded from their clinical research that out-of-hospital spinal immobilisation had little or no effect on the neurologic outcome in patients with blunt spinal injuries. In recent years, more publications have recommended limiting the use of the rigid spineboard. Schouten [13] formulated this contradiction: "spine boards are often mistakenly viewed as measures for providing spine stability; in fact, their purpose is to aid extrication and transfer" In a recent consensus paper [14], the authors showed that there is no consensus on the question whether injury mechanism can be used as a reliable predictor of spinal injury, and consequently which patient groups (if any) should be fully immobilised. They suggested that the spineboard should be used only as an extrication device. In the same year, Hauswald [15] published a position paper on the use of the spineboard stating that the use of rigid spineboard for spinal stabilisation during transport is irrational when examined from basic (physics) principles, and can be safely discarded. On the other hand, there is still a call from the field for some sort of spinal immobilisation, not only to protect the spine in patients with a high-energy impact during extrication and transportation [16], but also out of concern for potentially missed injuries and fear of reprisals and litigation [17]. Furthermore, the practice is deeply engrained in protocols and guidelines for the professionals [18], and laymen and 
victims have come to expect a spineboard or vacuum mattress being used after a highimpact injury. Acknowledging that the use of devices for external immobilisation in prehospital care will not disappear in the near future, it is time for a critical appraisal of the rigid spineboard and the vacuum mattress, and a search for possible alternatives.

\section{Protecting the spinal column using a spineboard \& headblocks or a vacuum mattress}

Although in daily practice the spineboard and vacuum mattress are intended to help prevent secondary damage to the already injured spine, direct evidence to support this notion is generally lacking $[12,15,19]$. Panjabi et al [20] showed that a stable spinal injury will remain stable as long as there are no forces adding to the physical load (i.e. the normal use of the spine without external forces such as a motor vehicle crash acting on the spine). This notion is supported by the clinical observations made by Benger et al [21], who showed that no ambulatory patient who self-presented to the Emergency Department with possible cervical spine injury, experienced sudden neurological deterioration which could be attributed to a lack of immobilisation. In addition, recent studies [22-24] have shown that controlled self-extrication leads to less motion of the cervical spine than spineboard-assisted extrication. This is in keeping with other studies that have shown that the risk of a spinal cord injury is much smaller with slow movements within the normal range of motion than in fast high-impact forces during the time of injury [25]. Also, the spinal cord is fairly flexible for movements within its physiological range of motion, and stiffens up significantly in pathological movements, so that greater force is needed to achieve these detrimental movements [25].

A number of cadaver studies have evaluated the amount of force needed to induce damage to the spine. Although this type of study cannot be directly translated into daily practice (e.g. due to the absence of physiological myogenic responses which help stabilise the spine [25]), these studies, along with finite element computer simulation, have provided valuable insights into the forces needed to cause spinal injury. Results from these studies indicate that forces of $300 \mathrm{~N}$ and more exerted directly onto the spine [5,26-28] are needed to induce damage to the intact spine, depending on circumstances (magnitude and direction of the force, shape of the spine, personal factors such as disease, and condition of the spine [25,28-30]. Other studies [31] have indicated that rotation forces increase the risk of spinal column damage. In general, when the normal range of motion is exceeded, the excess energy can cause tissue destruction [15]. Thus, as long as movements do not exceed resistance, handling 
the patient will not damage the spine. No studies are available concerning the forces needed to increase damage in an unstable injured spine.

\section{Advantages and disadvantages of using a spineboard or vacuum mattress}

There are some practical benefits for the health care providers (nurses, physicians) in using a spineboard or vacuum mattress [15,32]. A patient on a spineboard can be handled more easily, which can be an advantage for instance when the patient needs to be turned onto their side in case of vomiting. Transfer from the gurney to a hospital bed or CT scan Table is facilitated by a spineboard. Other aids, such as a slide board (e.g. Patslide) to facilitate such transfers have been developed, although they require more personnel to keep to the patient in line. Also, a spineboard or vacuum mattress is a clear visual clue that the patient may have a spinal injury. This can result in the spine being handled with greater care during trauma resuscitation performed according to ATLS guidelines [11].

Several authors have advocated the use of the spineboard in trauma patients [15,33-35], while at the same time suggesting that the time a patient spends on the spineboard should be limited and that the patient should be removed from the spineboard as soon as possible [11,36], preferably on arrival at the Emergency Department $[19,37,38]$. However, extended and prolonged use of the spineboard has been encountered in daily practice [16,39]. In addition, the authors of the Cochrane Review on spinal immobilisation [40] did not find any randomised controlled trials on the use of spineboards and concluded that the effect of spinal immobilisation on mortality, neurologic injury, and spinal stability remains uncertain. Two updates after this first publication reported no conclusive evidence concerning the efficacy of using a spineboard to prevent further spinal injury [41,42].

In fact, a large number of studies have shown the disadvantages of the spineboard or vacuum mattress, ranging from problems due to being restrained in a supine position to problems due to lying on a rigid surface. Several studies have shown that lying supine and restricted on a spineboard leads to increased breathing effort [43-45], and that these effects increase in time [43]. If patients are already at risk for breathing difficulties due to their injuries, lying supine may leave them more susceptible to oxygenation problems and infections [46-48]. Furthermore, lying supine carries the risk of aspiration [49], and is associated with higher intracranial pressures compared to elevated head positions [50-52]. Increased intracranial pressure can damage brain tissue, and contribute to the shifting of brain structures and brain herniation, as well as the induction of hydrocephalus by restricting blood supply to the brain [53]. 
Finally, the risk of cervical spine injury in many trauma patients requires that the head and neck of the patient are immobilised in the anatomical or "neutral" position, rather than aligning the airway axes by flexing the neck and extending the head into the "sniffing" position. This anatomical positioning of the patient hampers airway management [54], so more time and more attempts are needed for successful intubation [55].

Specific problems also arise from the fact that the spineboard has a rigid surface which does not follow the curvature of the human body [33]. It is especially in young children $[13,56,57]$, who have a relatively large head, that positioning on a flat surface can result in anterior translation and flexion of a cervical injury [56]. In patients with spinal deformities, it may be impossible to achieve a flat horizontal position on a rigid spineboard. Forcing a patient with spinal malformations into a cervical orthosis and then taping the head down on the spineboard could lead to further displacement in an extension-distraction injury, with potentially catastrophic consequences $[13,33,58]$.

\section{Options for prehospital spinal immobilisation}

There are basically three methods to place a patient on a rigid spineboard, with a trade-off between the number of movements that need to be made with the patient and the number of personnel needed. The most widely accepted practice is the log-roll method (Figure 1.2a) [11,35,59]. This method involves rolling a supine patient onto his side, placing the spineboard up against the patient's back, and then turning the patient and spineboard simultaneously into the supine position [60,61] (Figure 1.2a). Alternative options are the "lift-and-slide" technique (Figure 1.2b) and the $6+1$ person lift technique (Figure 1.2c), which result in less movement of the spine compared to log-rolling [62-64]. However, these techniques are much less incorporated in daily routines because of the number of personnel needed.
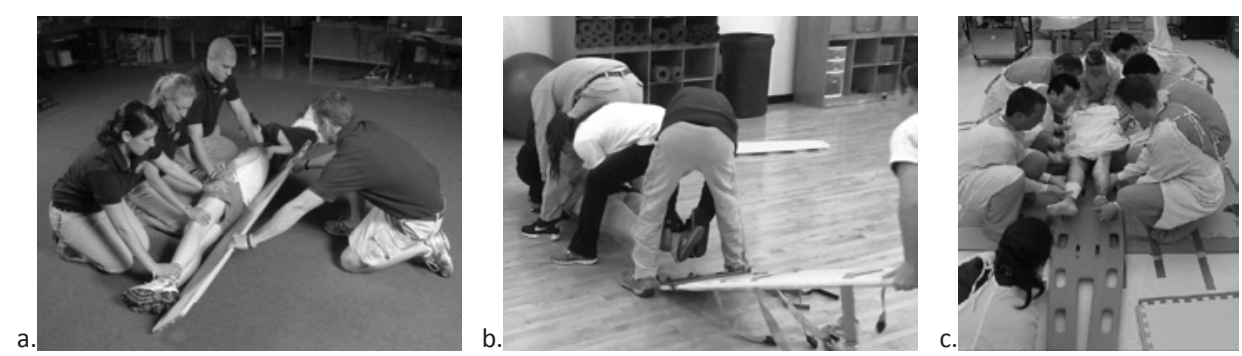

Figure 1.2a-c. Methods to place a patient on a rigid spineboard. a. log roll; b. lift and slide; c. 6+1 person lift

As an alternative to the rigid spineboard, the scoop stretcher (Figures 1.3a and 1.3b) and vacuum mattress (Figure 1.3c) can be used (usually together) for extrication and 
transport. The scoop stretcher is rigid, and consists of two halves that can be laterally slid underneath the patient. After the two halves have been fastened, the patient can be placed on a vacuum mattress or be carried away from the site of the accident. As with the lift-and-slide technique, this technique results in less rotational movement than the log-roll [62]. After the patient has been placed on a vacuum mattress and is securely fastened, the mattress is evacuated, resulting in the patient lying on a hard surface [19]. However, although seemingly rigid, the mattress needs to be supported to prevent sagging. This can be done for example by placing the mattress on a scoop stretcher. Because the mattress can be folded around the body, immobilisation is superior to that achieved using a spineboard, both objectively [62, 65-68] and subjectively $[66,68]$.

a.

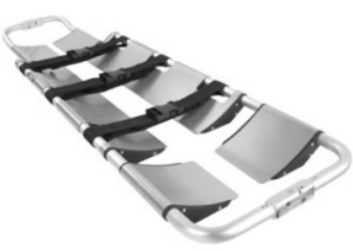

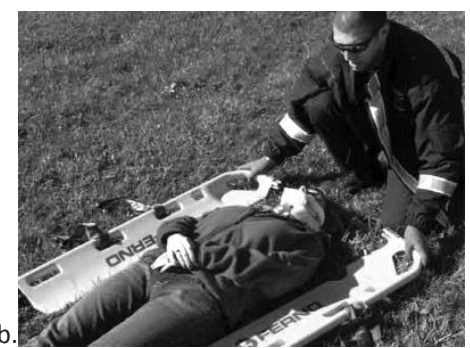

c.

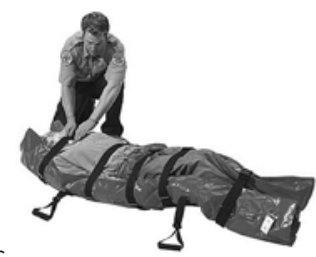

Figure 1.3a-c. Devices for prehospital spinal immobilisation. a. scoop stretcher; b: scoop stretcher; $\mathbf{c}$ : vacuum mattress

\section{The importance of the surface of the device}

Lying on a rigid surface means that the soft tissues of the body (skin, muscles, fat) are deformed so as to adapt to the shape of the surface (Figure 1.4). Lying on a rigid surface is not only uncomfortable [66,69-73], it also compromises the skin and underlying soft tissues. High tissue-interface pressures may also lead to closure of the capillaries, thereby depriving tissues of oxygen and nutrients [74]. Although there is no consensus on the exact threshold for capillary closure pressure [75-77], concerns that the pressures exerted by the spineboard and vacuum mattress exceed this threshold are established $[78,79]$. 


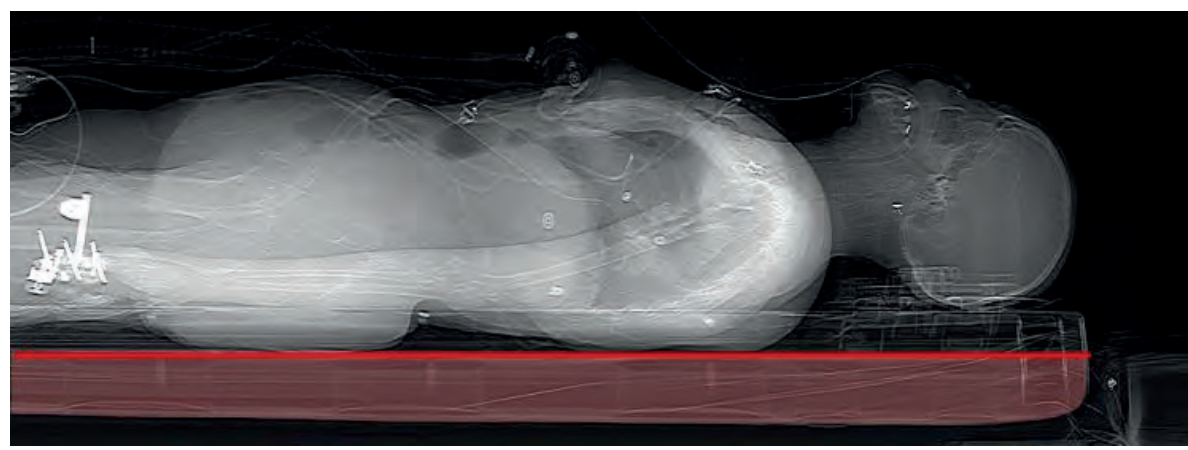

Figure 1.4. CT scan showing deformation of the buttocks caused by lying on a rigid spineboard.

Furthermore, the cells are subjected to strain and shear forces, which can, as an additional factor to pressure, induce cellular damage [80, 81]. Oxygen/nutrient deprivation and cell damage together may lead to tissue necrosis, manifesting itself as pressure ulcers $[72,75,82]$. Pressure ulcers are painful [83-85] and debilitating for the patient [86,87] and take a long time to heal [88-90], resulting in prolonged hospitalisation [91,92], and reduced quality of life [93-96].

The most effective way to prevent pressure ulcers is to reduce the amount of pressure or the time during which the tissues are exposed to the high pressures/shear forces $[75,97]$. This can be achieved by placing the patient on a softer surface in the first place, by relieving pressure via repositioning or by removing the patient from the surface as soon as possible. Removing the patient from the spineboard or vacuum mattress is the most effective way to relieve the high tissue-interface pressures, although it is unclear what constitutes a safe duration of immobilisation on a hard surface from a pressure ulcer point of view [37]. In addition, there is reluctance to remove spinal immobilisation before spinal injuries have been ruled out $[39,98,99]$. Relieving tissue-interface pressures by repositioning is not a realistic option while the patient is lying on a spineboard. Therefore, the solution should be sought in the hardness of the surface the patient is lying on. Lying on a softer surface, which allows the soft tissues to maintain their natural shape, preventing occlusion and shear forces, could be an important step forward in preventing pressure ulcers.

There are several ways to investigate the impact of forces applied to the human body. Invasive methods such as microdialysis can provide information about a range of biological changes, but evoke a local inflammatory response which influences the measurement of local inflammatory indicators [100]. A widely used non-invasive technique is to evaluate tissue-interface pressures [78,79,101-104], providing detailed geographic data on the pressures on the body surface, although this yields no information about the actual biological effect of these pressures. Finite element calculations based on magnetic resonance imaging (MRI) can provide data concerning 
tissue deformation due to the pressures applied [105-107], although providing limited information concerning actual tissue damage. Recently, studies have been published on non-invasive measurement of biological indicators of tissue damage caused by pressure [108-110]. The inflammatory response could yield potential biomarkers, such as cytokines, which are released after mechanical injury of the skin cells [111]. The proinflammatory cytokine IL1a is released in the skin in response to mechanical stimulus/ injury of the keratinocytes. Whether IL1a is a waste product from dysfunctional cells or whether it should be regarded as an indicator of a normal response to tissue stress [112] remains to be determined. In any case, it is released after pressure loading of the skin [77], and is increased in the skin in category I pressure ulcers [109], and could thus be used as an objective indicator of tissue response to pressure.

The combination of tissue-interface pressure mapping, MRI imaging and cytokine IL1a measurement was therefore used in the studies presented in this thesis to obtain an overall insight into the physical response to pressure.

\section{The soft-layered spineboard}

The spineboard, vacuum mattress and scoop stretcher have advantages in prehospital care for patients with suspected spinal injuries, but all also have specific drawbacks. The spineboard is a useful tool to aid extrication, but it has its limitations as regards immobilisation. The vacuum mattress, although superior in following the contours of the patient's body, carries the risk of loss of vacuum, thereby also losing it immobilising capacities. The use of the scoop stretcher produces the smallest amount of movement, but it cannot be used to extricate a patient from a car wreck. Furthermore, all three devices have a rigid surface, thereby potentially compromising the soft tissues. Consequently, an alternative extrication and immobilisation device which addresses these issues may provide benefits [33,72,113].

We therefore developed a so-called soft-layered long spineboard (Figure 1.5). This device is intended for both extrication and immobilisation, while avoiding the disadvantages associated with a rigid surface.

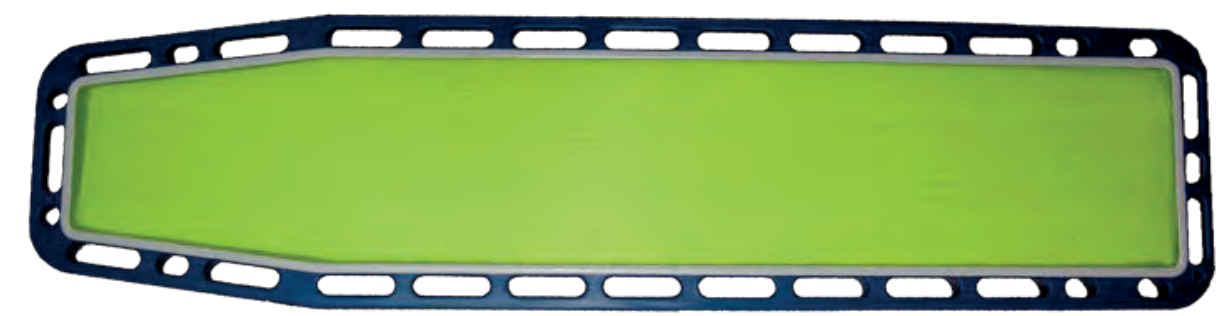

Figure 1.5. The soft-layered spineboard. 
Any new device for transport of patients in an ambulance must conform to the regulations described in European standards (NEN 1865 [114]), which aim to ensure the safety of the patient and the persons working with the device. Another important requirement regards the radiological properties of the device. In practice, removal from the device is often postponed until after radiographs have been made $[37,39,99]$. Thus, spine and other X-rays and/or CT-scans are frequently made while the patient is on the spineboard or vacuum mattress [39], making the radiolucency properties of the device very important [115]. Inadequate material choices can hamper interpretation of the images $[116,117]$, and may even imply radiographs having to be redone with the device removed in order to obtain good quality images. This is not only timeconsuming and costly, it also exposes the patient to extra radiation. Furthermore, if the patient is still on the device when the radiographs are made, the device will absorb part of the radiation. Current $\mathrm{x}$-ray machines compensate for this phenomenon by automatically increasing the radiation dose to ensure good quality radiographs (automatic exposure control; AEC). Since there is a relationship between increased radiation dosages and increased risk of cancer [118-121] due to DNA damage [122124], it is important that the benefits of radiation exposure are weighed critically against its risks. In accordance with the ALARA (as low as reasonably achievable) concept in radiography to minimise the radiation dosage [125], the materials placed between $\mathrm{x}$-ray source and $\mathrm{x}$-ray detector should therefore have a low level of absorption.

\section{Objective and outline of the thesis}

To investigate the value of the soft-layered spineboard in comparison to the existing rigid spineboard and vacuum mattress, a number of studies comparing these three devices was performed. The main hypothesis of this thesis is that the soft-layered spineboard compares favourably to the rigid spineboard and vacuum mattress in terms of patient comfort, tissue-interface pressures, tissue deformation and radiological properties. Chapter 2 reports on a study comparing the comfort and tissue-interface pressures for the three devices. The tissue-interface pressure exerted by the devices on the body was measured in 30 healthy, awake volunteers. The study reported on in Chapter 3 investigated the effect of anaesthesia in patients on the rigid or soft-layered spineboard. This study aimed to answer the question whether loss of muscle tone and function leads to a different pattern of tissue-interface pressures compared to the awake status. A group of anaesthetised patients was used as a proxy for comatose or paralysed trauma patients, which form the most important target group for spinal immobilisation. Chapter 4 reports on a study in which MRI scans of the sacral area 
were made in healthy volunteers on all three devices, combined with a computer simulation model, finite element analysis, to estimate the amount of tissue deformation induced by the three devices, as a first indicator of tissue damage. In Chapter 5, findings are presented of a study comparing cytokine level profiles in healthy, awake volunteers on the soft-layered spineboard and rigid spineboard, to examine the immunological tissue response to pressure. Finally, Chapters 6 and 7 discuss radiological issues concerning spinal immobilisation. These studies aimed to establish which of the three devices has the best radiological properties from a point of view of respectively producing artefacts and radiological dose absorption.

In summary, the main questions addressed in this thesis are:

- Which device for prehospital immobilisation is the most comfortable?

- Which device for prehospital immobilisation results in the lowest tissue-interface pressures?

- Which device for prehospital immobilisation leads to the least tissue deformation?

- Which device for prehospital immobilisation results in the lowest expression of tissue damage markers?

- Which device for prehospital immobilisation has the best radiological properties? 


\section{References}

1. Warden CA. Splint stretcher board. USA patent 2141100. 1938.

2. Crutchfield WG. Fracture-dislocations of the cervical spine. Am J Surg. 1937;XXXVIII:592-8.

3. Moir JL. First-aid treatment for fractured spines. Br Med J. 1942;Oct 31:529.

4. Thomson JEM. First aid and transportation of suspected spine injuries. Am J Surg. 1939;XLV:42-7.

5. Furst $W$. The force required to crush vertebrae: its probable mechanical relation to the postmetrazol fracture. Psychiat Quart. 1940;14:397-402.

6. Virgin WJ. Experimental investigations into the physical properties of the intervertebral disc. J Bone Joint Surg. 1951;33B:607-11.

7. Farrington JD. Death in a ditch. Bull Am Coll Surg. 1967;98(6):44-53.

8. Farrington JD. Extrication of victims - surgical principles. J Trauma. 1968;8(4):493-512.

9. Geisler WO, Wynne-Jones M \& Jousse AT. Early management of the patient with trauma to the spinal cord. Med Serv J Can. 1966;22:512-23.

10. Bez U, \& Buhren W. Vacuum mattress, especially for rescue vehicles USA patent US4234982A. 1980.

11. American College of Surgeons, ed. Advanced Trauma Life Support. Student course manual, 9th ed. 2012: Chicago, IL.

12. Hauswald M, Ong G, Tandberg D \& Omar Z. Out-of-hospital spinal immobilization: its effect on neurologic injury. Acad Emerg Med. 1998;5:214-9.

13. Schouten R, Albert $T$ \& Kwon BK. The spine-injured patient: initial assessment and emergency treatment. J Am Acad Orthop Surg. 2012;20:336-46.

14. Connor D, Greaves I, Porter K, \& Bloch M. Pre-hospital spinal immobilisation: an initial consensus statement. Emerg Med J. 2013;30:1067-9.

15. Hauswald M. A re-conceptualisation of acute spinal care. Emerg Med J. 2013;30:720-3.

16. Bouland AJ, Jenkins JL, \& Levy MJ. Assessing attitudes toward spinal immobilization. J Emerg Med. 2013;45:e117-e25.

17. Morrissey JF, Kusel ER, \& Sporer KA. Spinal motion restriction: an educational and implementation program to redefine prehospital spinal assessment and care. Prehosp Emerg Care. 2014; 18:429-432.

18. Deasy C, \& Cameron P. Routine application of cervical collars - What is the evidence? Injury. 2011;42:841-2.

19. Lubbert PHW, Schram ME \& Leenen LPH. Is there a reason for spine board immobilization in the Emergency Department for patients with a potential spinal injury? Eur J Trauma. 2005;31:375-8.

20. Panjabi MM, White AA, and Johnson RM. Cervical spine mechanics as a function of transection of components. J Biomech. 1975;8:327-36.

21. Benger J, \& Blackham J. Why do we put cervical collars on conscious trauma patients? Scan J Trauma Resus Emerg Med. 2009;17:44-7.

22. Dixon M, O'Halloran J, \& Cummins NM. Biomechanical analysis of spinal immobilisation during prehospital extrication: a proof of concept study. Emerg Med J. 2013; doi:10.1136/emermed-2013202500

23. Shafer JS, \& Naunheim RS. Cervical spine motion during extrication: a pilot study. West J Emerg Med. 2009;10:74-8.

24. Engsberg JR, Standeven JW, Shurtleff TL, Eggars JL, Shafer JS \& Naunheim RS. Cervical spine motion during extrication. J Emerg Med. 2013;44:122-7.

25. Cusick JF, \& Yoganandan N. Biomechanics of the cervical spine 4: major injuries. Clin Biomech. 2002;17:1-20.

26. Tchako A, \& Sadegh A. A cervical spine model to predict injury scenarios and clinical instability. Sports Biomech. 2009;8:78-95. 
27. Bauze RJ, \& Ardran GM. Experimental production of forward dislocation in the human cervical spine. J Bone Joint Surg. 1978;60B:239-45.

28. Shea M, Edwards WT, White AA \& Hayes WC. Variations of stiffness and strength along the human cervical spine. J Biomech. 1991;24:95-107.

29. Maiman DJ, Yoganandan N \& Pintar FA. Preinjury cervical alignment affecting spinal trauma. J Neurosurg-Spine. 2002;97:57-62.

30. Nightingale RW, McElhaney JH, Richardson WJ, Best TM \& Myers BS. Experimental impact injury to the cervical spine: relating motion of the head and the mechanism of injury. J Bone Joint Surg. 1996;78A:412-21.

31. Roaf R. A study of the mechanics of spinal injuries. J Bone Joint Surg. 1960;42B:810-23.

32. Mazolewski P, \& Manix TH. The effectiveness of strapping techniques in spinal immobilization. Ann Emerg Med. 1994;23:1290-5.

33. White CC DR, Millin MG and the Standards and Clinical Practice Committee, National Association of EMS Physicians. EMS spinal precautions and the use of the long backboard - resource document to the position statement of the national association of EMS physicians and the American College of Surgeons committee on trauma. Prehosp Emerg Care. 2014;18:306-14.

34. Theodore N, Hadley MN, Aarabi B, et al. Prehospital cervical spinal immobilization after trauma. Neurosurgery. 2013;72:22-34.

35. Moss R, Porter K \& Greaves I. Minimal patient handling: a faculty of prehospital care consensus statement. Emerg Med J. 2013;30:1065-6.

36. Porter KM, \& Allison KP. The UK emergency department practice for spinal board unloading. Is there conformity? Resuscitation. 2003;58:117-20.

37. Ahn H, Singh J, Nathens A, et al. Pre-hospital care management of a potential spinal cord injured patient: a systematic review off the literature and evidence-based guidelines. J Neurotrauma. 2011;28:1341-61.

38. Vickery D. The use of the spinal board after the pre-hospital phase of trauma management. Emerg Med J. 2001;18:51-4.

39. Stagg MJ, \& Lovell ME. A repeat audit of spinal board usage in the emergency department. Injury. 2008;39:323-6.

40. Kwan I, Bunn F \& Roberts IG. Spinal immobilisation for the trauma patient. Cochrane database of systematic reviews. 2001. doi: 10.1002/14651858.CD002803.

41. Kwan I, \& Bunn F. Effects of prehospital spinal immobilization: a systematic review of randomized trials on healthy subjects. Prehosp Disaster Medicine. 2005;20(1):47-53.

42. Kwan I, Bunn F Roberts IG. Spinal immobilisation for trauma patients (review). Cochrane Database Syst Rev. 2009.

43. Ay D, Aktas C, Yesilyurt S, Sarikaya S, Cetin A, \& Ozdogan ES. Effects of spinal immobilization devices on pulmonary function in healthy volunteer individuals. Ulus Travma Acil Cerrahi Derg (Turkish Journal of Trauma and Emergency Surgery). 2011;17:103-7.

44. Bauer D, \& Kowalski R. Effect of spinal immobilization devices on pulmonary function in the healthy, nonsmoking man. Ann Emerg Med. 1988;17(9):915-8.

45. Walsh M, Grant T, \& Mickey S. Lung function compromised by spinal immobilization. Correspondence. Ann Emerg Med. 1990;19:615-6.

46. Voggenreiter G, Aufmkolk M, Stiletto RJ, et al. Prone positioning improves oxygenation in posttraumatic lung injury - a prospective randomized trial. J Trauma. 2005;59:333-43.

47. Beuret P, Carton M-J, Nourdine K, Kaaki M, Tramoni G \& Ducreux J-C. Prone position as prevention of lung injury in comatose patients: a prospective, randomized, controlled study. Intensive Care Med. 2002;28:564-9.

48. Hale DF, Cannon JW, Batchinsky Al, et al. Prone positioning improves oxygenation in adult burn patients with severe acute respiratory distress syndrome. J Trauma Acute Care Surg. 2012;72:1634-9. 
49. Brownlee AE. Managing spinal immobilisation devices in the emergency department. Australas Emerg Nurs J. 2005;8:79-83.

50. Meixensberger J, Baunach S, Amschler J, Dings J \& Roosen K. Influence of body position on tissue-pO2, cerebral perfusion pressure and intracranial pressure in patients with acute brain injury. Neurol Rese. 1997;19:249-53.

51. Ng I, Lim J \& Wong HB. Effects of head posture on cerebral hemodynamics; its influences on intracranial pressure, cerebral perfusion pressure and cerebral oxygenation. Neurosurgery. 2004;54:593-8.

52. Agbeko RS, Pearson SBS, Peters MJ, McNames J, \& Goldstein B. Intracranial pressure and cerebral perfusion pressure responses to head elevation changes in pediatric traumatic brain injury. Pediatr Crit Care Med. 2012;13:e39-e47.

53. Graham DI, \& Gennareli TA. Pathology of brain damage after head injury. In: Cooper P, \& Golfinos G, editor. Head injury, 4th ed. New York: Morgan Hill; 2000.

54. Doran JV, Tortella BJ, Drivet WJ \& Lavery RF. Factors influencing successful intubation in the prehospital setting. Prehosp Disaster Medicine. 1995;10:259-64.

55. Pinchalk M, Roth RN, Paris PM, \& Hostler D. Comparison of times to intubate a simulated trauma patient in two positions. Prehosp Emerg Care. 2003;7:252-7.

56. Herzenberg JE, Hensinger RN, Derick DK \& Philips WA. Emergency transport and positioning of young children who have an injury of the cervical spine. The standard backboard may be hazardous. J Bone Joint Surg Am. 1989;71:15-22.

57. Nypaver M, \& Treloar D. Neutral cervical spine positioning in children. Ann Emerg Med. 1994;23:208 211.

58. Hofman M, Janzing HMJ \& Brink PRG. Gaat u maar even plat liggen meneer...(article in Dutch). Ned Tijdschr Trauma. 2007;6:191-4.

59. Conrad BP, Del Rossi G, Horodyski M, Prasarn ML, Alemi Y \& Rechtine GR. Eliminating log rolling as a spine trauma order. Surg Neurol Int. 2012;3:S188-S97.

60. Conrad BP, Horodyski M, Wright J, Ruetz P \& Rechtine GR. Log-rolling technique producing unacceptable motion during body position changes in patients with traumatic spinal cord injury. J Neurosurg Spine. 2007;6:540-3.

61. McGuire RA, Neville S, Green BA \& Watts C. Spinal instability and the log-rolling maneuver. J Trauma. 1987;27:525-31.

62. Del Rossi G, Rechtine GR, COnrad BP \& Horodyski M. Are scoop stretchers suitable for use on spineinjured patients? Am J Emerg Med. 2010;28:751-6.

63. Del Rossi G, Horodyski M, Bryan C, DiPaola CP, DiPaola MJ \& Rechtine GR. Transferring patients with thoracolumbar spinal instability: are there alternatives to the log roll maneuver? Spine. 2008;33:1611 -1615 .

64. Horodyski M, Conrad BP, Del Rossi G, DiPaola CP \& Rechtine GR. Removing a patient from the spine board: is the lift and slide safer than the log roll? J Trauma. 2011;70:1282-5.

65. Prasarn ML, Zhou H, Dubose D, et al. Total motion generated in the unstable thoracolumbar spine during management of the typical trauma patient: a comparison of methods in a cadaver model. J Neurosurg-Spine. 2012;16:504-8.

66. Hamilton RS, \& Pons PT. The efficacy and comfort of full-body vacuum splints for cervical-spine immobilization. J Emerg Med. 1996;14:553-9.

67. Prasarn $M L$, Horodyski $M$, Dubose $D$, et al. Total motion generated in the unstable cervical spine during management of the typical trauma patient. Spine. 2012;37:937-42.

68. Krell JM, McCoy MS, Sparto PJ, Fisher GL, Stoy WA \& Hostler DP. Comparison of the Ferno scoop stretcher with the long backboard for spinal immobilization. Prehosp Emerg Care. 2006;10:46-51.

69. Cross DA, \& Baskerville J. Comparison of perceived pain with different immobilization techniques. Prehosp Emerg Care. 2001;5:270-4. 
70. Cordell WH, Hollingsworth JC, Olinger ML, Stroman SJ \& Nelson DR. Pain and tissue-interface pressures during spine-board immobilization. Ann Emerg Med. 1995;26:31-6.

71. Hauswald M, Hsu M \& Stockoff C. Maximizing comfort and minimizing ischemia: a comparison of four methods of spinal immobilization. Prehosp Emerg Care. 2000;4:250-2.

72. Luscombe MD, \& Williams JL. Comparison of a long spinal board and vacuum mattress for spinal immobilisation. Emerg Med J. 2003;20:476-8.

73. Chan D, Goldberg R, Tascone A, Harmon S \& Chan L. The effect of spinal immobilization on healthy volunteers. Ann Emerg Med. 1994;23(1):48-51.

74. Berg G, Nyberg S, Harrison P, Baumchen J, Gurss E \& Hennes E. Near-infrared spectroscopy measurement of sacral tissue oxygen saturation in healthy volunteers immobilized on rigid spine boards. Prehosp Emerg Care. 2010;14:419-24.

75. DeFloor T. The risk of pressure sores: a conceptual scheme. J Clin Nurs. 1999;8:206-16.

76. Landis E. Micro-injection studies of capillary blood pressure in human skin. Heart. 1930;15:209-28.

77. Bronneberg D, Spiekstra SW, Cornelissen LH, et al. Cytokine and chemokine release upon prolonged mechanical loading of the epidermis. Exp Dermatol. 2007;16:567-573.

78. Keller BPJA, Lubbert PHW, Keller E \& Leenen LPH. Tissue-interface pressures on three different support-surfaces for trauma patients. Injury. 2005;36:946-8.

79. Sheerin F, \& de Frein R. The occipital and sacral pressures experienced by healthy volunteers under spinal immobilization: a trial of three surfaces. J Emerg Nurs. 2007;33:447-50.

80. Oomens CWJ, Loerakker S, \& Bader DL. The importance of internal strains as opposed to interface pressures in the prevention of pressure related deep tissue injury. J Tissue Viabil. 2010;19:35-42.

81. Loerakker S, Manders E, Strijkers GJ, et al. The effects of deformation, ischemia, and reperfusion on the development of muscle damage during prolonged loading. J Appl Physiol. 2011;111:1168-77.

82. Mak AFT, Yu Y, Kwan LPC, Sun L \& Tam EWC. Deformation and reperfusion damages and their accumulation in subcutaneous tissues during loading and unloading: a theoretical modeling of deep tissue injuries. J Theor Biol. 2011;11:65-73.

83. Pieper B, Langemo D \& Cuddigan J. Pressure ulcer pain: a systematic literature review and National Pressure Ulcer Advisory Panel white paper. Ostomy Wound Manag. 2009;55:16-31.

84. Rastinehad D. Pressure ulcer pain. J Wound Ostomy Cont. 2006;33:252-7.

85. Gunes UY. A descriptive study of pressure ulcer pain. Ostomy Wound Manag. 2008;54:56-61.

86. Hopkins A, Dealey C, Bale S, DeFloor T \& Worboys F. Patient stories of living with a pressure ulcer. J Adv Nurs. 2006;56:345-53.

87. Fox C. Living with a pressure ulcer: a descriptive study of patients' experiences. Br J Community Nurs. 2002;7:10-22.

88. Bennett G DCPJ. The cost of pressure ulcers in the UK. Age Ageing. 2004;33:230-5.

89. Chapman BR, Mills KJ, Pearce LM, \& Crowe TC. Use of an arginine-enriched oral nutrition supplement in the healing of pressure ulcers in patients with spinal cord injuries: an observational study. Nutr \& Diet. 2011;68:208-13.

90. Sanada H, lizaka S, Matsui Y, et al. Clinical wound assessment using DESIGN-R total score can predict pressure ulcer healing: pooled analysis from two multicenter cohort studies. Wound Repair Regen. 2011;19:559-67.

91. Graves N, Birrell FA, \&Whitby M. Modeling the economic losses from pressure ulcers among hospitalized patients in Australia. Wound Repair Regen. 2005;13:465-7.

92. Allman RM, Damiano AM \& Strauss MJ. Pressure ulcers, hospital complications, and disease severity: impact on hospital costs and length of stay. Adv Skin Wound Care. 1999;12:22-30.

93. Langemo DK, Melland H, Hanson D, Olson B \& Hunter S. The lived experience of having a pressure ulcer: a qualitative analysis. Adv Skin Wound Care. 2000;13:225-35.

94. Spilsbury K, Nelson A, Cullum N, Iglesias C, Nixon J \& Mason S. Pressure ulcers and their treatment and effects on quality of life: hospital inpatient perspectives. J Adv Nurs. 2007;57:494-504. 
95. Essex HN, Clark M, Sims J, Warriner A \& Cullum N. Health-related quality of life in hospital inpatients with pressure ulceration: assessment using generic health-related quality of life measures. Wound Repair Regen. 2009;17:797-805.

96. Gorecki C, Brown JM, Nelson EA, et al. Impact of pressure ulcers on quality of life in older patients: a systematic review. J Am Geriatr Soc. 2009;57:1175-83.

97. Reddy M, Gill SS, \& Rochon PA. Preventing pressure ulcers: a systematic review. JAMA. 2006;296:974 984.

98. Tello RR, Braude D, Fullerton L \& Froman P. Outcome of trauma patients immobilized by emergency department staff, but not by emergency medical service providers: a quality assurance initiative. Prehosp Emerg Care. 2014;18:544-549.

99. Hauswald M, \& Braude D. Diffusion of medical progress: early spinal immobilization in the emergency department. Acad Emerg Med. 2007;11:1087-9.

100. Stenken JA, Church MK, Gill CA \& Clough GF. How minimally invasive is microanalysis sampling? A cautionary note for cytokine collection in human skin and other clinical studies. The AAPS Journal. 2009;12(1):73-8.

101. Thompson-Bishop JY, \& Mottola CM. Tissue interface pressure and estimated subcutaneous pressures of 11 different pressure-reducing support surfaces. Adv Skin Wound Care. 1992;5:42-8.

102. Rondorf-Klum LM, \& Langemo D. Relationship between body weight, body position, support surface, and tissue interface pressure at the sacrum. Adv Skin Wound Care. 1993;6:22-31.

103. Blaylock B, \& Gardner C. Measuring tissue interface pressures of two support surfaces used in the operating room. Ostomy Wound Manag. 1994;40:42-4.

104. Hickerson WL, Slugocki GM, Thaker RL, Duncan R, Bishop JF \& Parks JK. Comparison of total body tissue interface pressure of specialized pressure-relieving mattresses. J Long-term Eff Med. 2004; 14:14-27.

105. Linder-Ganz E, Shabshinb N, Itzchak Y, Gefen A. Assessment of mechanical conditions in sub-dermal tissues during sitting: a combined experimental-MRI and finite element approach. J Biomech. 2007;40:1443-54.

106. Stekelenburg A, Oomens CWJ, Strijkers GJ, de Graaf LAHJ, Bader DL \& Nicolay K. A new MR-compatible loading device to study in-vivo muscle damage development in rats due to compressive loading. Med Eng Phys. 2006;28:331-8.

107. Shabshin N, Ougortsin V, Zoizner G \& Gefen A. Evaluation of the effect of trunk tilt on compressive soft tissue deformations under the ischial tuberosities using weight-bearing MRI. Clin Biomech. 2010;25:402-8.

108. Cornelissen LH. Modeling the transport of biochemical markers in skin. Towards pressure ulcer risk assessment. PhD thesis. Chapter 5: The transport profile of cytokines in compromised human skin. Eindhoven, the Netherlands: Eindhoven University of Technology; 2008.

109. Bronneberg D. Biochemical markers for early detection of superficial pressure ulcers. PhD Thesis. Chapter 6: In vivo detection of grade I pressure ulcers. Eindhoven, the Netherlands: Eindhoven University of Technology; 2007.

110. Perkins MA, Osterhues MA, Farage MA \& Robinson MK. A noninvase method to assess skin irritation and compromised skin conditions using simple tape adsorption of molecular markers of inflammation. Skin Res Technol. 2001;7:227-37.

111. Delavary BM, van der Veer WM, van Egmond M, Niessen FB \& Beelen RHJ. Macrophages in skin injury and repair. Immunobiology. 2011;216(7):753-62.

112. Bronneberg D, Bouten CVC, Oomens CWJ, van Kemenade PM \& Baaijens FPT. An in vitro model system to study the damaging effects of prolonged mechanical loading of the epidermis. Ann Biomed Eng. 2006;34:506-14.

113. Sundstrom T, Asbjornsen H, Habiba S, Sunde GA \& Wester K. Prehospital use of cervical collars in trauma patients: a critical review. J Neurotrauma. 2014;31:531-40. 
114. Netherlands Standards Institute [Nederlands Normalisatie-instituut]. Specificaties voor brancards en andere middelen voor het vervoer van patienten in ambulances [in Dutch; Specifications for gurneys and other devices for transport of patients in ambulances]. Delft, the Netherlands 1999.

115. Loewenhardt $B$, Huttinger $R$, Reinert $M$, et al. Dose effects and image quality: is there any influence by bearing devices in whole-body computed tomography in trauma patients? Injury. 2014;45:170-5.

116. Miller JA, Mele C, \& Abu-Judeh H. Significance of backboard artifacts on portable trauma series chest radiographs. Emerg Radiol. 1999;6:334-8.

117. Schou J, Kiermayer H, Ummenhofer W \& Herion H-P. In search of the most suitable technique for trunctal spinal immobilization with associated radiography. Eur J Emerg Med. 2001;8:89-92.

118. Berrington de Gonzalez A, \& Darby S. Risk of cancer from diagnostic x-rays: estimates for the UK and 14 other countries. Lancet. 2004;363:345-51.

119. Einstein AJ, Henzlova MJ \& Rajagopalan S. Estimating Risk of Cancer Associated With Radiation Exposure From 64-Slice Computed Tomography Coronary Angiography. JAMA. 2007;298(3):317-23.

120. Hall EJ, \& Brenner DJ. Cancer risks from diagnostic radiology. British J Radiol. 2008;81:362-78.

121. Shimizu Y, Schull WJ \& Kato H. Cancer risk among atomic bomb survivors. The RERF Life Span Study. JAMA. 1990;264(5):601-4.

122. Rothkamm K, \& Lobrich M. Evidence for a lack of DNA double-strand break repair in human cells exposed to very low x-ray doses. P Natl Acad Sci USA. 2003;100:5057-62.

123. Pogribny I, Koturbash I, Tryndyak V, et al. Fractionated low-dose radiation exposure leads to accumulation of DNA damage and profound alterations in DNA and histone methylation in the murine thymus. Mol Cancer Res. 2005;3:553-61.

124. Little JB. Chapter 14: Ionizing radiation. In: Kufe DW, Pollock RE, Weichselbaum RR, et al, editor. Cancer Medicine (6th ed). Hamilton, Ont: B.C. Decker; 2000.

125. International Commission on Radiological Protection. Recommendations of the International Commission on Radiological Protection. Annals of the ICRP, Publication 60 (Oxford: Pergamon Press). 1991. 



\section{Reduced tissue-interface pressure and increased comfort on a newly developed soft- layered long spineboard}
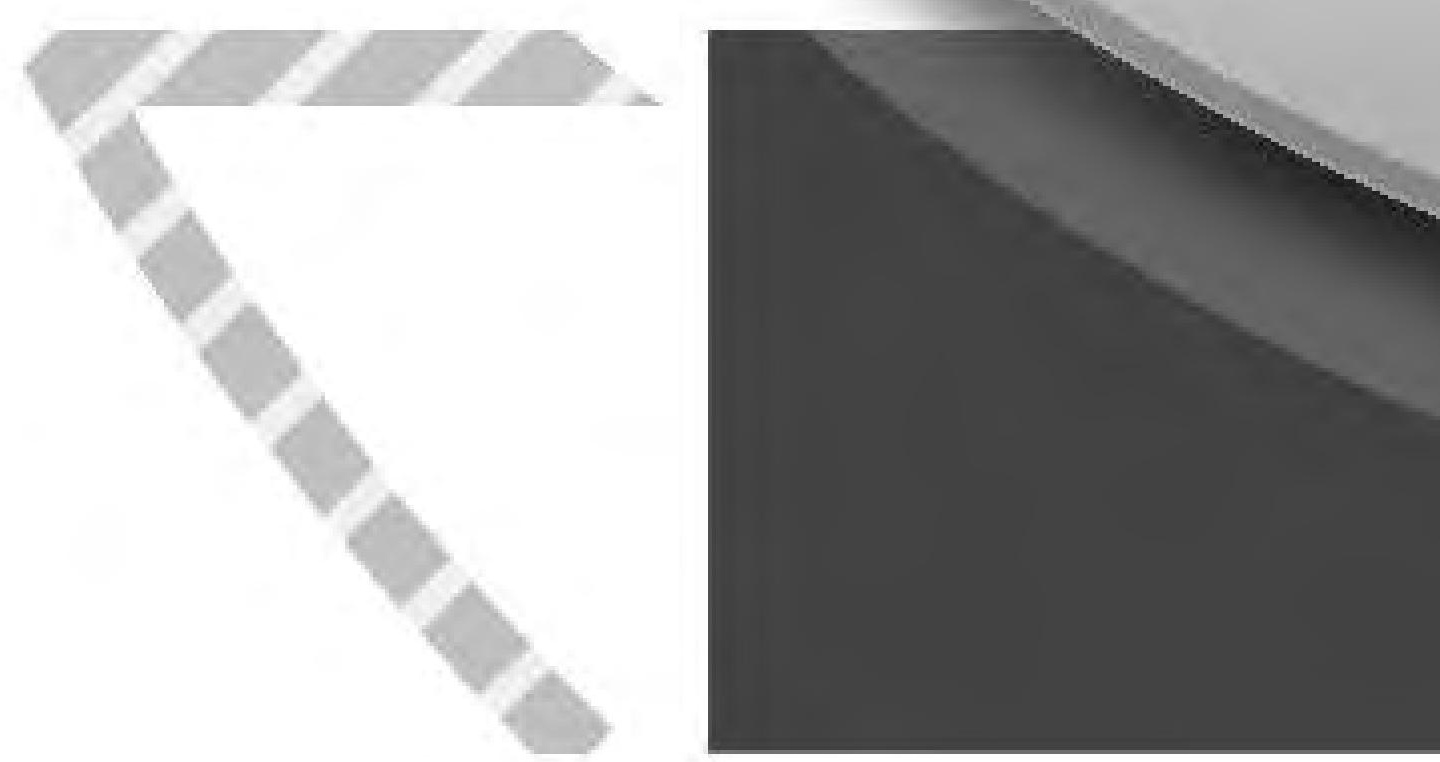

Baukje Hemmes, Martijn Poeze, Peter R.G. Brink

Journal of Trauma. 2010;68: 593-598 


\section{Abstract}

Background: Immobilisation of the spine in patients with trauma at risk of spinal damage is performed using a rigid long spineboard or vacuum mattress both during prehospital and in-hospital care. However, disadvantages of these immobilisation devices in terms of discomfort and tissue-interface pressures have guided the development of a new soft-layered long spineboard. We compared tissue-interface pressure and degree of discomfort during immobilisation on a rigid spineboard, a vacuum mattress, and a newly developed soft-layered long spineboard.

Methods: In this randomised crossover trial, 30 volunteers were immobilised sequentially on all three devices for 15 minutes per device. Tissue-interface pressures were measured using an Xsensor pressure mapping device, including the peak pressure and the Peak Pressure Index (PPI). Discomfort was rated on a Visual Analog Scale after 1 minute and after 15 minutes of immobilisation.

Results: Tissue-interface pressures were significantly higher on the standard long spineboard and the vacuum mattress than on the soft-layered long spineboard. PPI for the sacrum was close to peak pressure on both the spineboard and the vacuum mattress. PPI for the sacrum on the soft-layered long spineboard was significantly lower, with an average PPI close to normal diastolic blood pressures. The participants reported significantly less discomfort on the soft-layered long spineboard compared with the rigid long spineboard, both after 1 minute and 15 minutes $(p<0.001)$.

Conclusions: This study revealed a relevant reduction in tissue-interface pressures and discomfort when using a soft-layered long spineboard compared with using a standard rigid long spineboard or a vacuum mattress. Emergency care providers should consider the use of the soft-layered long spineboard to reduce the discomfort and potential tissue damage caused by immobilisation and transport on a rigid long spineboard or vacuum mattress. 


\section{Introduction}

Current methods of spinal immobilisation with long spineboards and cervical collar are well incorporated into both prehospital and in-hospital trauma protocols [1,2]. Spineboards are used to safely extricate patients with trauma from the scene of a crash and for transfer to the hospital. Although physicians are advised to remove patients from these boards as soon as possible [3-5], most patients undergo extensive evaluation in the emergency and/or computed tomography (CT) room to prevent them being removed from the spineboard before the spine is cleared $[6,7]$. In a substantial number of patients, this phase exceeds a period of 2 hours $[8,9]$ and the average duration is more than 1 hour [10].

Although spinal immobilisation is clearly needed to protect trauma patients with spinal injury, there is extensive evidence that prolonged immobilisation may cause significant discomfort, pain [11-14], and pressure ulceration over bony prominences [15-17], leading to prolonged hospital stay $[18,19]$ and increased cost of care $[20,21]$. The use of vacuum mattresses has been advocated by some to counteract these problems [22]. However, when negative pressure is applied to immobilise a trauma patient, the surface of the vacuum mattress is just as hard as that of the spineboard [23]. With these disadvantages in mind, a new product has been developed, which can be used for extrication and immobilisation, with a softer surface to prevent high tissueinterface pressures while at the same time maintaining the rigidity necessary to protect the patient's spine (Figure 2.1).

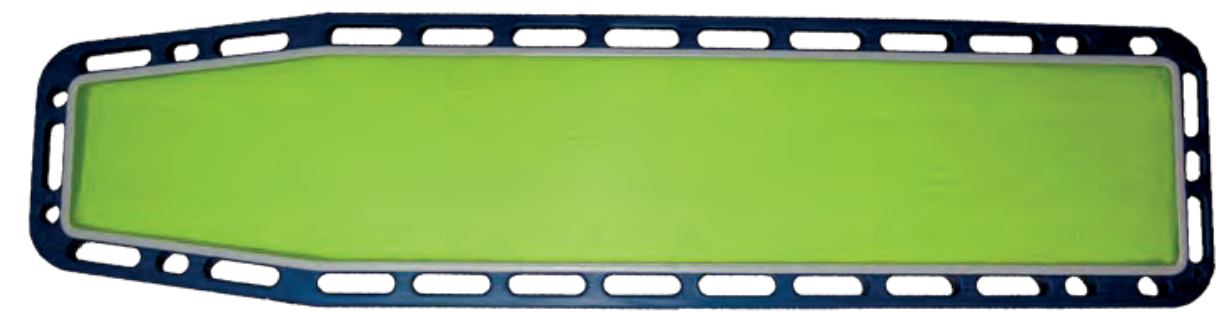

Figure 2.1. The newly developed soft-layered long spineboard. The soft-layered long spineboard has the same dimensions as a standard rigid long spineboard, but instead of a hard surface, a replaceable mattress is embedded in the frame. The mattress is shielded by a protective, flexible yet tough synthetic overlay.

In this study, we compared the newly developed soft-layered long spineboard with the standard long spineboard and the vacuum mattress in terms of tissue-interface pressures and the discomfort experienced during the immobilisation of healthy volunteers. Our main research hypothesis was that pressure exerted on critical body contact points would be lower when using the soft-layered long spineboard, compared with either the standard long spineboard or vacuum mattress, and that the comfort 
experienced would be greater with the soft-layered long spineboard than with the standard long spineboard or the vacuum mattress.

\section{Materials and Methods}

\section{Study Design}

We conducted a prospective, randomised, nonblinded, crossover, comparative study on tissue-interface pressure and discomfort using a standard long spineboard, a vacuum mattress, and a soft-layered long spineboard in healthy volunteers.

\section{Recruitment of Participants}

A sample of 30 healthy volunteers (19 men and 11 women) was recruited from the employees of the Maastricht University Medical Centre. These included emergency department (ED) nurses and physician assistants, surgeons, intensive care personnel, and administrative staff. All participants were free of discomfort and had no skin problems before participating. There was no financial compensation for participation. Approval was obtained from the Institutional Review Board of the Maastricht University Medical Centre. Written informed consent was obtained from all participants before they were enrolled in this study.

A power analysis was performed using the peak pressures found by Keller et al. [23]. We assumed that the peak pressures on the soft-layered long spineboard would be comparable with those found for emergency room overlay mattresses, which are considered to pose no additional risk of tissue damage. Power analysis showed that we should be able to detect significant differences between the three devices in terms of mean peak tissue-interface pressure on the critical body areas of interest (sacrum, 175 $\mathrm{mm} \mathrm{Hg}$ vs. $118 \mathrm{~mm} \mathrm{Hg}$; and scapulae, $177 \mathrm{~mm} \mathrm{Hg}$ vs. $90 \mathrm{~mm} \mathrm{Hg}$ for standard long spineboard vs. soft-layered long spineboard, respectively), with $95 \%$ reliability if we used 30 volunteers.

\section{Interventions}

Three different support devices designed for prehospital trauma care were tested: a standard long spineboard (Ferno-Washington, Wilmington, $\mathrm{OH}$ ), a vacuum mattress (RedVac, Radstadt, Austria), and a prototype soft-layered long spineboard. All participants were positioned on all three devices, by means of concealed random allocation.

A strap (Fastrap model 770; Ferno-Washington) was used to immobilise the subject on the standard long spineboard and the soft-layered long spineboard. Immobilisation on the vacuum mattress was achieved by applying negative pressure. 
These procedures ensured a realistic reflection of the body position of a patient with trauma during actual transport. Before the start of the measurement, the researcher made sure that the folding of the vacuum mattress and the strapping with the standard long spineboard and soft-layered long spineboard induced no discomfort in the participants.

The measurement started as soon as the participant was lying on the device, as if he or she was ready for transport (vacuum and straps applied for the vacuum mattress, and straps applied for the standard and soft-layered long spineboards). Each intervention lasted for 15 minutes, with 5-minute breaks between the interventions, during which the participants were encouraged to walk around. The period of 15 minutes is considered a realistic time in the Netherlands to transport a patient with trauma from the scene of the crash to the nearest ED. Furthermore, it was considered the shortest exposure time in which it should be possible to detect differences in pressure and comfort between the three devices.

\section{Measurements}

Tissue-interface pressures were continuously measured using a Xsensor X2-6912 pressure-mapping device (Xsensor Technology, Calgary, Canada). This system consists of a thin, easily foldable full-body pressure-mapping pad, equipped with 6,912 capacitive sensors. This pad was placed between the subject and the support surface, without folds. Pressure on the sensors generates a voltage difference, which increases linearly with the amount of pressure. We connected the pad to a laptop computer with special Xsensor software (version 4.2), for real-time pressure recording.

After the volunteers had lain on each device for 1 minute and 15 minutes, subjective comfort was measured by asking them to indicate comfort on a Visual Analog Scale from 10 (extreme discomfort) to 0 (extreme comfort). During the measurements, participants were allowed to wear their normal clothes, but no shoes. In addition, they were asked to empty the pockets of their trousers and loosen their hair. Participants were instructed to lie calmly but were allowed to talk during the measurements.

\section{Outcome Measures}

Pressures were recorded continuously. Peak pressures (in $\mathrm{mmHg}$ ) measured the occiput, the scapulae, the sacrum, and the heels were noted at the start of each measurement and subsequently after each minute. The average of all measurements during the 15-minute period (mean peak pressure) was calculated for each body region to compensate for motion effects.

The Peak Pressure Index (PPI) was calculated as a measure of pressure on a larger surface area.24 PPI was defined as the area of $3 \times 3$ sensors, which included the sensor 
showing the peak pressure and which had the highest average pressure including this sensor. The area was defined based on the first recording. The PPI can be considered more reliable than peak pressure for assessing the pressure-distributing capabilities of a support surface, because it examines a $14.5-\mathrm{cm} 2$ surface area in addition to the peak pressure registered by one single sensor, with a surface of only $1.61 \mathrm{~cm} 2$. PPI was calculated solely for the sacrum, because this is the only body region with a relatively flat bony structure, whereas the occiput, scapulae, and heels have relatively pointy bony structures. Moreover, the sacrum is the body region with the highest risk of developing pressure ulcers [25-27].

\section{Data Collection and Processing}

Statistical analysis was performed using the Statistical Package for Social Sciences (SPSS, Chicago, IL), version 12.0.1. Pressures are presented as mean \pm SD. Mean peak pressures and PPI were compared using repeated measures analysis of variance, with post hoc analysis (Bonferroni) for the sequence of devices tested. Subjective comfort scores were also assessed using repeated measures analysis of variance. Significance was assumed at $P<0.05$.

\section{Results}

\section{Characteristics of Study Subjects}

Table 2.1 presents the characteristics of the participants $(n=30)$ in this study. Mean height and weight were significantly greater for the men than for the women. Age and body mass index were not significantly different between men and women.

Table 2.1. Mean and standard deviation for main characteristics of the participants

\begin{tabular}{llll}
\hline & $\begin{array}{l}\text { Men } \\
(\mathrm{n}=19)\end{array}$ & $\begin{array}{l}\text { Women } \\
(\mathrm{n}=11)\end{array}$ & $\begin{array}{l}\text { Total } \\
(\mathrm{n}=30)\end{array}$ \\
\hline Age $($ years $)$ & $41 \pm 11$ & $36 \pm 12$ & $39 \pm 11$ \\
Height $(\mathrm{cm})^{*}$ & $183 \pm 6$ & $170 \pm 6$ & $178 \pm 9$ \\
Weight $(\mathrm{kg})^{*}$ & $85 \pm 9$ & $69 \pm 17$ & $79 \pm 15$ \\
Body mass index & $25.3 \pm 2.5$ & $23.7 \pm 4.4$ & $24.7 \pm 3.4$ \\
\hline
\end{tabular}

Data are presented as mean \pm SD.

$* t=5.942 ; d f=28, P<0.001$ for the difference between men and women

$\dagger t=3.376 ; d f=28, \mathrm{P}<0.005$ for the difference between men and women

\section{Tissue-Interface Pressures}

The pressure measurements were analysed independently by the first and second authors. Interrater correlation was high with $r^{2}=0.998(P<0.001)$. Interrater reliability was also high with $r^{2}=0.984(P<0.001)$. 
The soft-layered long spineboard produced significantly lower mean peak pressure at occiput and sacrum than the standard long spineboard and the vacuum mattress. Mean peak pressure on the scapulae was higher with the standard long spineboard than with either the soft-layered long spineboard or the vacuum mattress. Mean peak pressure on the heels was higher for the vacuum mattress than for the other two devices (Figure 2.2).

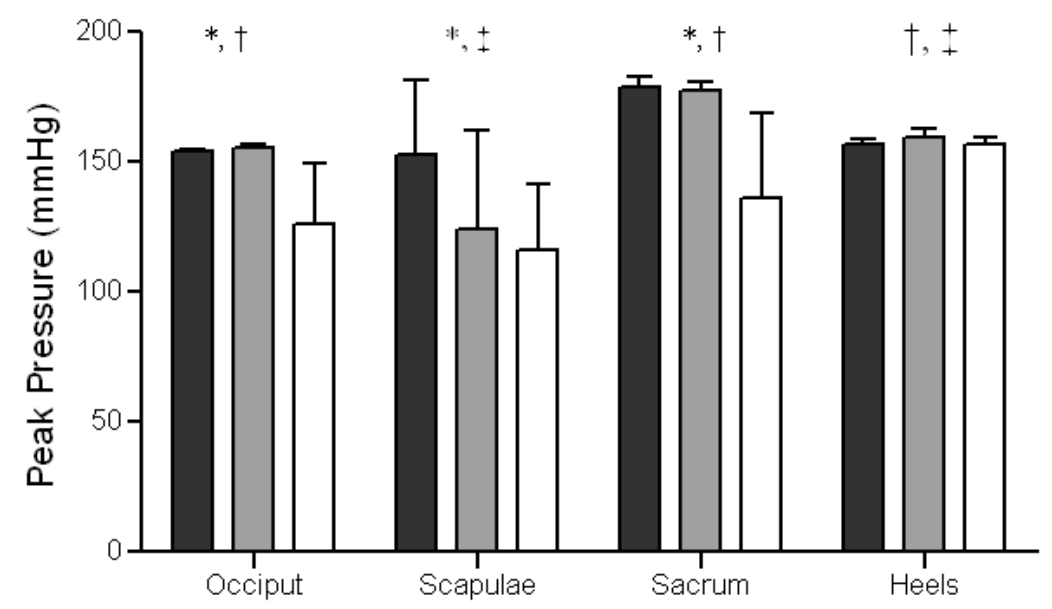

Figure 2.2. Mean peak pressure between contact site and support device. Mean peak pressures and SD for standard long spineboard (dark grey bars), vacuum mattress (light grey bars), and soft-layered long spineboard (white bars). Mean peak pressure differs between devices for all contact sites. ${ }^{*} \mathrm{P}<0.05$ by comparing standard long spineboard with soft-layered long spineboard; $+P<0.05$ by comparing soft-layered long spineboard with vacuum mattress; $\ddagger \mathrm{P}<0.05$ by comparing standard long spineboard with vacuum mattress.

Figure 2.3 shows the mean peak pressure and mean PPI values for the sacrum on the three different support devices. Both mean peak pressure and PPI at the sacrum were almost identical on the standard long spineboard and vacuum mattress and much lower on the soft-layered long spineboard $(F=48.2, P<0.001$ and $F=298.1, P<0.001$, respectively). 


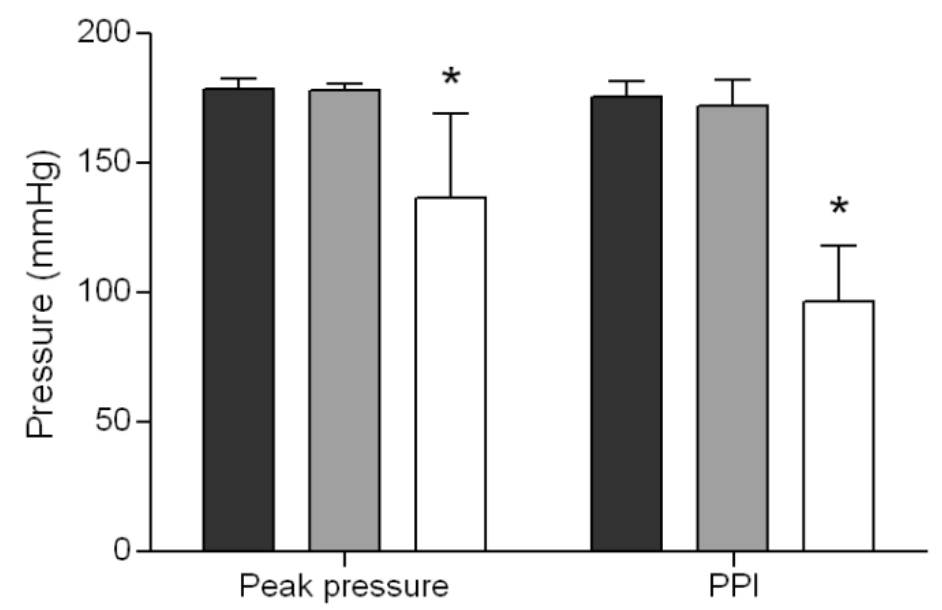

Figure 2.3. Mean and SD for peak pressure and PPI at the sacrum for three devices. Mean peak pressure and mean PPI at the sacrum were equally high for the standard long spineboard (dark grey bars) and vacuum mattress (light grey bars), and significantly lower for the soft-layered long spineboard (white bars).

$* P<0.001$ comparing soft-layered long spineboard with standard long spineboard and vacuum mattress.

Interestingly, the standard long spineboard and (to a lesser extent) the vacuum mattress showed lack of support for the normally lordotic lumbar spine. In contrast, the soft-layered long spineboard did give support to this part of the spine (data not shown).

\section{Comfort}

Figure 2.4 shows how the participants appreciated the level of comfort they perceived with the three devices. A score of 0 refers to maximum comfort, whereas a score of 10 refers to maximum discomfort. The participants reported significantly less discomfort on the soft-layered long spineboard compared with the standard long spineboard, both after 1 minute and 15 minutes $(F-=23.9, P<0.001)$. Differences between the soft-layered long spineboard and the vacuum mattress failed to reach significance $(F=$ $3.2, P<0.083)$ after 1 minute and 15 minutes. 


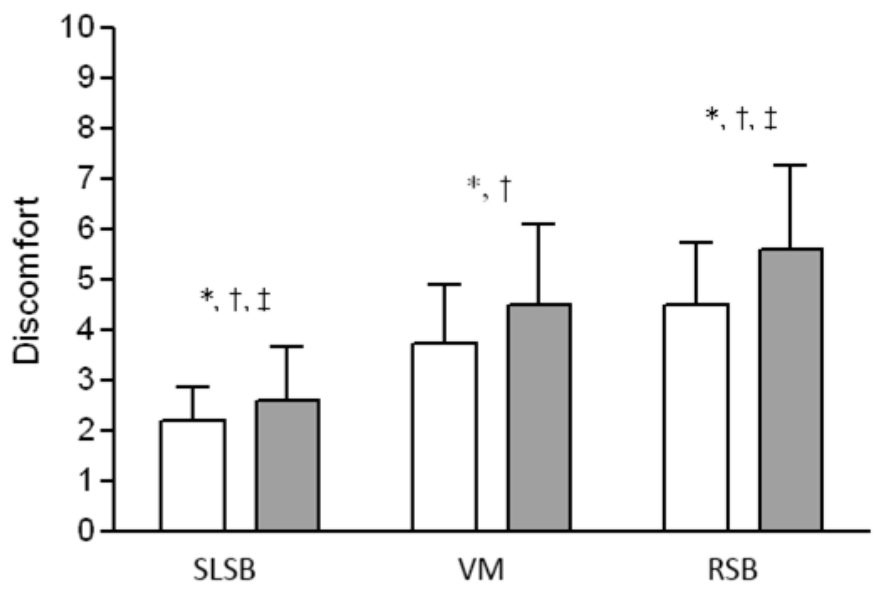

Figure 2.4. Mean subjective discomfort scores after 1 minute and 15 minutes. Discomfort experienced by participants increased over time and differed between devices. Data are presented as mean and SD of Visual Analog Scale comfort score. White bars represent discomfort after 1 minute; grey bars represent discomfort after 15 minutes. Discomfort was significantly lower on soft-layered long spineboard than on standard long spineboard and vacuum mattress and increased over time for all devices. SLSB: soft-layered spineboard; VM: vacuum mattress; RSB: rigid spineboard.

$* P<0.001$ for group effect, $+P<0.001$ for time-effect, $¥ P<0.001$ for interaction effect, using repeatedmeasures analysis of variance.

\section{Discussion}

Spinal immobilisation is widely considered the standard of care for trauma patients with potential spinal injuries. However, the disadvantages of using a standard long spineboard, such as high tissue-interface pressures and discomfort have received considerable attention and guided research into possible alternatives [28-33]. In this study, we compared a newly developed device, the soft-layered long spineboard, with two existing devices (the standard long spineboard and the vacuum mattress) for prehospital extrication, immobilisation, and transport. High tissue-interface pressures were found for both the standard long spineboard and the vacuum mattress, whereas the soft-layered long spineboard was associated with lower tissue-interface pressures and greater comfort in healthy volunteers.

Transport to a trauma centre and diagnostic procedures may take a long time to complete, during which the high tissue-interface pressures caused by the standard long spineboard increase the risk of skin damage. Reswick and Rogers [34] showed that lying on a hard surface leads to an unacceptable risk of developing pressure ulcers in less time than lying on a soft surface. Other authors [22,35] suggested using of a 
vacuum mattress as an alternative to the standard long spineboard. Despite the improved comfort observed when using the vacuum mattress, this device carries the risk of loss of vacuum during extrication and transport, thereby posing a great risk to a potentially damaged spine. Luscombe and Williams [35] therefore, concluded that "no single system appears to provide the ideal for extrication and transport of a trauma casualty." The soft-layered long spineboard proved to produce lower tissue-interface pressure and greater comfort than both the standard spineboard and vacuum mattress, which makes it an interesting alternative to these devices.

Preventing pressure ulcers can be achieved in various ways, including pressure reduction and redistribution. To examine both these processes, we not only measured peak pressures but also the PPI, which is the mean pressure over a $14.5-\mathrm{cm} 2$ area [24]. The PPI values for the sacrum on both the standard long spineboard and the vacuum mattress were close to peak pressure, indicating that the peak pressure is not redistributed over the contact site. This leaves areas of high pressure over the sacrum, which is the body region most susceptible to developing pressure ulcers [25-27]. The soft-layered long spineboard, on the other hand, had both lower peak pressure and lower PPI for the sacrum, indicating better pressure reduction and pressure redistribution over the sacrum, compared with the other two devices.

Although pressure mapping technology has proved to be a reliable and valid method to assess pressure distribution and tissue-interface pressure [36], no consensus has been reached about the critical perfusion pressure of tissues in relation to the development of pressure ulcers. Critical pressures as low as $32 \mathrm{~mm} \mathrm{Hg}$ for capillaries have been reported [37] but debated [16]. According to Defloor [16], the diastolic blood pressure would be a more correct critical pressure for tissue damage. In a healthy volunteer, this would mean that external pressures over $80 \mathrm{~mm} \mathrm{Hg}$ can cause tissue damage. A recent in vitro study with skin cultures showed that the first signs of tissue damage can be found at externally applied pressures as low as $50 \mathrm{~mm} \mathrm{Hg}$ [38]. Although it is unclear which pressures lead to clinically relevant tissue damage, it is clear that there is a trade-off between tissue pressure and the time that elapses before tissue damage occurs $[34,39]$. Therefore, using the soft-layered long spineboard, which imposes less pressure on the tissue than the standard long spineboard and the vacuum mattress, means buying time to optimise the patient's treatment while minimising tissue damage.

Participants judged the soft-layered long spineboard to be the most comfortable of the three devices tested. Despite the objectively measured pressures on the vacuum mattress being equally high as those on the standard long spineboard, the vacuum mattress was considered more comfortable than the long spineboard. Earlier studies on pain-related Visual Analog Scale scores indicate that a difference of about $30 \%$ is relevant to patients [40-42]. Differences in comfort between the soft-layered long 
spineboard and the other two devices at both 1 minute and 15 minutes of up to $113 \%$ in favour of the soft-layered long spineboard are therefore clinically relevant.

A number of comments need to be made on the interpretation of our observations. First, this study was undertaken in optimal circumstances with healthy volunteers. Our population of volunteers was, thus, not a true representation of all trauma patients, because our participants were all healthy and awake. Although weight and body mass index of the participants were representative of the Dutch population, they may not be representative of other populations. Second, our participants lay on the three devices for only 15 minutes each. In the Netherlands, it is almost always possible for an emergency service to get from the collision site to an ED within 15 minutes. On arrival at the ED, the trauma patient could theoretically be removed from the device immediately. We, therefore, considered 15 minutes to be the minimum time any person should be able to lie on a device without developing tissue damage or discomfort because of the device used, while at the same time revealing any significant differences in pressure and comfort. In actual practice, time on the spineboard can easily be longer [8-10]. More time spent on any device may influence the comfort experienced [11], which will probably lead to more pronounced differences in discomfort between the devices. Furthermore, we did not use any direct measurements of potential tissue damage caused by the devices. No visual inspection of the skin in the contact areas was undertaken. Further studies should focus on the relation between local tissue pressure and irreversible tissue damage.

In conclusion, this randomised crossover trial found tissue-interface pressures and comfort experienced by 30 healthy volunteers to be significantly better when volunteers were immobilised on a soft-layered long spineboard than on the standard long spineboard and vacuum mattress. Tissue-interface pressures on the vacuum mattress and the standard long spineboard were found to be equally high. Emergency care providers should consider the use of a soft-layered long spineboard to reduce the discomfort and potential tissue damage caused by immobilisation and transport on a standard long spineboard or vacuum mattress. 


\section{References}

1. American College of Surgeons Committee on Trauma. Advanced Trauma Life Support for Doctors. 7th ed. Chicago, IL: American College of Surgeons; 2004.

2. National Association of Emergency Medical Technicians. Prehospital Trauma Life Support. 6th ed. St.Louis, MO: Mosby/JEMS Publications; 2006.

3. Vickery D. The use of the spinal board after the pre-hospital phase of trauma management. Emerg Med J. 2001;18:51-54.

4. Lubbert PHW, Schram ME \& Leenen LPH. Is there a reason for spine board immobilization in the Emergency Department for patients with a potential spinal injury? Eur J Trauma. 2005;31:375-378.

5. Brownlee AE. Managing spinal immobilisation devices in the emergency department. Australas Emerg Nurs J. 2005;8:79-83.

6. Cooke MW. Use of the spinal board within the accident and emergency department. Emerg Med J. 1998;15:108 -113.

7. Stagg MJ \& Lovell ME. A repeat audit of spinal board usage in the emergency department. Injury. 2008;39:323-326.

8. Yeung JHH, Cheung NK, Graham CA, et al. Reduced time on the spinal board-effects of guidelines and education for emergency department staff. Injury. 2006;37:53-56.

9. Malik MH \& Lovell ME. Current spinal board usage in emergency departments across the UK. Injury. 2003;34:327-329.

10. Lerner EB \& Moscati R. Duration of patient immobilization in the ED. Am J Emerg Med. 2000;18:28 30.

11. Cordell WH, Hollingsworth JC, Olinger ML, et al. Pain and tissue-interface pressures during spineboard immobilization. Ann Emerg Med. 1995;26:31-36.

12. Zlupko GR, Megargel RE \& O'Connor RE. Patient discomfort due to indiscriminant use of spinal immobilization. [Abstract]. Prehosp Emerg Care. 2004;8:101.

13. Reddy M, Keast D, Fowler E \& Sibbald RG. Pain in pressure ulcers. Ostomy Wound Manage. 2003;49 (4 suppl):30 -35.

14. Rastinehad D. Pressure ulcer pain. J Wound Ostomy Cont. 2006;33:252-257.

15. Baldwin KM \& Ziegler SM. Pressure ulcer risk following critical traumatic injury. Adv Skin Wound Care. 1998;11:168 -173.

16. Defloor T. The risk of pressure sores: a conceptual scheme. J Clin Nurs. 1999;8:206 -216.

17. Watts D, Abrahams E, MacMillan C, et al. Insult after injury: pressure ulcers in trauma patients. Orthop Nurs. 1998;17:84 -91.

18. Graves N, Birrell FA \& Whitby M. Modeling the economic losses from pressure ulcers among hospitalized patients in Australia. Wound Repair Regen. 2005;13:462- 467.

19. Allman RM, Damiano AM \& Strauss MJ. Pressure ulcers, hospital complications, and disease severity: impact on hospital costs and length of stay. Adv Skin Wound Care. 1999;12:22-30.

20. Severens JL, Habraken JM, Duivenvoorden S \& Frederiks CM. The cost of illness of pressure ulcers in the Netherlands. Adv Skin Wound Care. 2002;15:72-77.

21. Bennett G, Dealey C \& Posnett J. The cost of pressure ulcers in the UK. Age Ageing. 2004;33:230 -235.

22. Chan D, Goldberg RM, Mason J, Chan L. Backboard versus mattress splint immobilization: a comparison of symptoms generated. J Emerg Med. 1996;14:293-298.

23. Keller BP, Lubbert PH, Keller E \& Leenen LP. Tissue-interface pressures on three different support surfaces for trauma patients. Injury. 2005;36:946 -948.

24. Keller BPJA, van Overbeeke J \& van der Werken C. Interface pressure measurement during surgery: a comparison of four operating Table surfaces. J Wound Care. 2006;15:5-9. 
25. Amlung SR, Miller WL \& Bosley LM. The 1999 National Pressure Ulcer Prevalence Survey: a benchmarking approach. Adv Skin Wound Care. 2001;14:297-301.

26. Vangilder C, Macfarlane GD \& Meyer S. Results of nine international pressure ulcer prevalence surveys: 1989 to 2005. Ostomy Wound Manage. 2008;54:40 -54.

27. Vanderwee KM, Clark M, Dealey C, Gunningberg L \& DeFloor T. Pressure ulcer prevalence in Europe: a pilot study. J Eval Clin Pract. 2007;13:227-235.

28. Kwan I \& Bunn F. Effects of prehospital spinal immobilization: a systematic review of randomized trials on healthy subjects. Prehosp Disaster Med. 2001;20:47-53.

29. Hauswald M \& Braude D. Spinal immobilization in trauma patients: is it really necessary? Curr Opin Crit Care. 2002;8:566 -570.

30. Stroh G \& Braude D. Can an out-of-hospital cervical spine clearance protocol identify all patients with injuries? An argument for selective immobilization. Ann Emerg Med. 2001;37:609-615.

31. Hauswald M, Ong G, Tandberg D \& Omar Z. Out-of-hospital spinal immobilization: its effect on neurologic injury. Acad Emerg Med. 1998;5:214 -219.

32. Domeier RM, Swor RA, Evans RW, et al. Multicenter prospective validation of prehospital clinical spinal clearance criteria. J Trauma. 2002;53:744 -750.

33. Hankins DG, Rivera-Rivera EJ, Ornato JP, et al. Spinal immobilization in the field: clinical clearance criteria and implementation. Prehosp Emerg Care. 2001;5:88 -93.

34. Reswick J \& Rogers J. Experience at the Rancho Los Amigos Hospital with devices and techniques to prevent pressure sores. In: Reswick J, Rogers J, eds. Bed Sore Mechanics. London: MacMillan Press; 1976: 301-310.

35. Luscombe MD \& Williams JL. Comparison of a long spinal board and vacuum mattress for spinal immobilization. Emerg Med J. 2003;20: 476-478.

36. Stinson MD, Porter-Armstrong AP \& Eakin PA. Pressure mapping systems: reliability of pressure map interpretation. Clin Rehabil. 2003;17:504-511.

37. Landis E. Micro-injection studies of capillary blood pressure in human skin. Heart. 1930;15:209 -228.

38. Bronneberg D, Spiekstra SW, Cornelissen LH, et al. Cytokine and chemokine release upon prolonged mechanical loading of the epidermis. Exp Dermatol. 2007;16:567-573.

39. Linder-Ganz E, Engelberg S, Scheinowitz M \& Gefen A. Pressure-time cell death threshold for albino rat skeletal muscles as related to pressure sore biomechanics. J Biomech. 2006;39:2725-2732.

40. Jensen MP, Chen C \& Brugger AM. Interpretation of visual analog scale ratings and change scores: a reanalysis of two clinical trials of postoperative pain. J Pain. 2003;4:407-414.

41. Farrar JT, Young JP, LaMoreaux, Werth JL \& Poole RM. Clinical importance of changes in chronic pain intensity measures on an 11-point numerical rating scale. Pain. 2001;94:149 -158.

42. Cepeda MS, Africano JM, Polo R, Alcala R \& Carr DB. What decline in pain intensity is meaningful to patients with acute pain? Pain. 2003;105:151-157. 



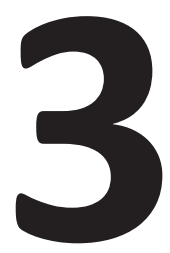

\section{Effects of unconsciousness during spinal immobilisation on tissue- interface pressures}

A randomised controlled trial comparing a standard rigid spineboard with a newly developed soft-layered long spineboard

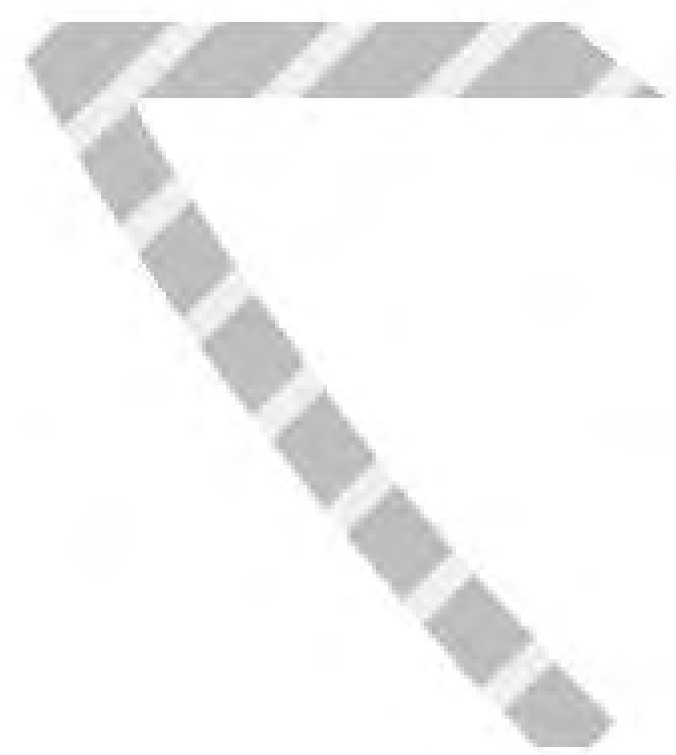

Baukje Hemmes, Martijn Poeze, Peter R.G. Brink Injury. 2014;45: 1741-1746 


\section{Abstract}

Background: Immobilisation of the spine of patients with trauma at risk of spinal damage is usually performed using a rigid long spineboard or vacuum mattress, both during prehospital and in-hospital care. However, disadvantages of these immobilisation devices in terms of discomfort and tissue-interface pressures have guided the development of soft-layered long spineboards. We compared tissue-interface pressures between awake and anaesthetised (unconscious) patients during immobilisation on a rigid spineboard and a soft-layered long spineboard.

Methods: In this comparative study, 30 anaesthetised patients were randomised to immobilisation on either the rigid spineboard or the soft-layered spineboard for the duration of their elective surgery. Tissue-interface pressures measured using an Xsensor pressure-mapping device were compared with those of 30 healthy volunteers who were immobilised sequentially on the rigid spineboard and the soft-layered spineboard. Redness of the sacrum was also recorded for the anaesthetised patients immediately after the surgery.

Results: For both anaesthetised patients and awake volunteers, tissue-interface pressures were significantly lower on the soft-layered spineboard than on the rigid spineboard, both at start and after $15 \mathrm{~min}$. On the soft-layered spineboard, tissue interface pressure and peak pressure index (PPI) for the sacrum were significantly lower for anaesthetised patients than for awake volunteers. Peak pressures and PPI on the rigid spineboard were equal for both groups. Tissue-interface pressures did not change significantly over time. Redness of the sacrum was significantly more pronounced on the rigid spineboard than on the soft-layered spineboard.

Conclusions: This prospective randomised controlled trial shows that using a softlayered spineboard compared to a rigid spineboard for spinal immobilisation resulted in lower tissue-interface pressures in both awake volunteers and anaesthetised patients. Moreover, tissue-interface pressures on the soft-layered spineboard were lower in anaesthetised patients than in awake volunteers. These findings show the importance of using a soft-layered spineboard to reduce tissue-interface pressure, especially for patients who cannot relieve pressure themselves by changing position. 


\section{Background}

Spinal immobilisation with long spineboards and cervical collars for extrication and transfer of trauma patients is a standard precaution in both prehospital and in-hospital protocols [1]. Especially for unconscious trauma patients, who are unable to maintain spinal alignment by muscular tone, these methods are considered essential to protect the spine from further injury.

Although there is a general consensus that the patient should be removed from the spineboard as soon as reasonably possible, the average time patients are reported to spend on a spineboard has been estimated to be around $1 \mathrm{~h}[2,3]$. There is extensive evidence, however, that prolonged immobilisation causes significant discomfort and pain, and on occasion may cause pressure ulceration over bony prominences, leading to prolonged hospital stay and increased cost of care [4-12].

Pressure ulceration occurs as a result of reduced perfusion due to prolonged pressure and/or shear injury to the tissues. Increased pressure can lead to compression of the dermal capillaries. When the pressure is relieved, reactive hyperaemia, clinically seen as a bright flush or reddened area that blanches under light pressure, can occur. At this stage of so-called blanchable erythema, damage to the underlying tissues has not yet occurred [13]. Shear injury occurs as the result of sliding, when the soft tissues are stretched relative to each other. The process of shear injury can occur quickly and the first signs of cell damage can be seen within minutes. In a recent study we demonstrated that when using a rigid spineboard, the maximum strains on the tissues can exceed the threshold for deformation damage [14]. Healthy conscious subjects may adopt pressure relieving strategies, involving position changes, to minimise the risk of exceeding this threshold at specific body regions. However, unconscious patients are thought not to adopt these strategies and may therefore be at higher risk of developing pressure induced tissue damage.

Therefore, this study investigated the hypothesis that when lying on a rigid spineboard, unconsciousness results in higher tissue-interface pressure compared to the awake status. In addition, a recent comparative study reported significantly lower interface pressures on a prototype spine board with soft, covered inlay [10]. Since the soft inlay may compensate for the lack of movement during unconsciousness, our second hypothesis was that there would be no difference in tissue-interface pressure between anaesthetised and awake status when lying on the soft-layered long spineboard. 


\section{Methods}

\section{Study design}

We conducted a prospective, randomised, single-blinded, comparative study on tissueinterface pressures and redness of the skin using a standard rigid long spineboard and a soft-layered long spineboard in patients undergoing surgery under general anaesthesia. Tissue interface pressures were compared with those of a group of awake healthy volunteers. The study has been assigned ISRCTN96064657.

\section{Recruitment of participants}

A sample of 30 patients ( 25 men and 5 women) was recruited at the outpatient department of the Maastricht University Medical Centre. Patients included were scheduled for open abdominal wall hernia repair under general anaesthesia, with an expected duration of less than $2 \mathrm{~h}$. Patients with pressure ulceration either at time of inclusion or in the medical history, or with pain other than that due to the operation indication were excluded. Approval was obtained from the Medical Research Ethics Committee of the Maastricht University Medical Centre. Written informed consent was obtained from all participants before they were enrolled in the study.

A power analysis was performed using the peak pressures found previously [10] on the standard rigid spine board and the soft-layered long spineboard in awake and healthy volunteers. The power analysis showed that if we included 12 patients in each of the conditions, we should be able to detect a $25 \%$ increase in peak pressure index (PPI, see below) between awake and unconscious patients on the soft-layered spine board (mean PPI 171 (SD 33)) with an alpha error of 0.05 and a beta error of 0.2.

\section{Interventions}

Two different support devices designed for prehospital trauma care were tested: a standard long spineboard (Ferno-Washington, Wilmington, $\mathrm{OH}$ ), and a prototype softlayered long spineboard. After induction of anaesthesia, patients were randomly assigned to one of the two devices. The pressure-mapping mat was placed on the device and the patient was then placed on the device. Pressure was recorded during the entire surgery. The control group consisted of awake healthy volunteers who were randomly put on each device for $15 \mathrm{~min}$, with an interval between the devices of 5 min, while tissue-interface pressures were recorded continuously.

\section{Measurements}

Tissue-interface pressures were continuously measured using an Xsensor X2-6912 pressure-mapping device (Xsensor Technology, Calgary, Canada). This system consists 
of a thin, easily foldable full-body pressure-mapping pad, equipped with 6912 capacitive sensors. This pad was placed between the subject and the support surface, without folds. Pressure on the sensors generates a voltage difference, which increases linearly with the pressure. We connected the pad to a laptop computer with special Xsensor software (version 4.2), for real-time pressure recording.

Directly after the surgery, visual inspection and semi-quantitative grading of the skin redness of the patient's back was performed. Any redness was tested for being blanchable using a clear plastic disc, so as to distinguish between blanchable and nonblanchable erythema [15].

\section{Outcome measures}

Pressures were recorded continuously from the start of the anaesthesia until the end of the surgical procedure and reported as described previously [10]. Briefly, peak pressures (in $\mathrm{mmHg}$ ) measured at the scapulae, the sacrum, and the heels were recorded continuously. The Peak Pressure Index (PPI) was calculated as a measure of pressure on a larger surface area [16]. PPI was defined as the area of $3 \times 3$ sensors (14.5 $\mathrm{cm} 2$ surface area) which included the sensor showing the peak pressure (one sensor $=1.61 \mathrm{~cm} 2$ ) and which had the highest average pressure including this sensor. The area was defined based on the first recording. PPI was calculated solely for the sacrum, because this is the only body region with a relatively flat bony structure, whereas scapulae and heels have relatively pointy bony structures. Moreover, the sacrum is the body region with the highest risk of developing pressure ulcers [17-19]. As a clinical measure of these tissue-interface pressures, the redness was scored postoperatively semi-quantitatively as absent, diffuse or clearly defined, and further categorised in terms of blanchable or non-blanchable.

\section{Data collection and processing}

Statistical analysis was performed using the Statistical Package for Social Sciences (SPSS, IBM), version 20.0.0.1. Pressures are presented as mean \pm SD. Analysis of tissueinterface pressures involved repeated-measures analysis of variance with correction of the degrees of freedom using the Greenhouse-Geisser estimates of sphericity when Mauchly's test indicated that the assumption of sphericity had been violated, using time as the within-group factor and treatment as the between-group factor. Post hoc group comparisons at the different time points were only performed when the overall repeated-measures tests were statistically significant. All scores were tested for normality using the Kolmogorov-Smirnov test. Parametric variables were compared using Student's t-test, while non-parametric variables were compared using the MannWhitney $U$ statistic. Nominal variables were compared across independent groups 
using the chi-squared test or Fisher's exact test. Homogeneity of variance was assessed using Levene's test. Level of significance was set at $P<0.05$.

\section{Results}

\section{Patients}

Characteristics of anaesthetised subjects and awake controls are described in Table 1. No differences in demographics or baseline characteristics were found between the patients randomised for rigid spineboard or soft-layered spineboard. Participants in the patient group were on average older and more often male than in the awake volunteers group. No relationship was found between pressure values and age or gender.

Table 3.1. Subject characteristics.

\begin{tabular}{lllll}
\hline & Anaesthetised & Anaesthetised & Awake & $\boldsymbol{P}^{*}$ \\
\hline Device & Rigid spineboard & $\begin{array}{l}\text { Soft-layered } \\
\text { spineboard }\end{array}$ & $\begin{array}{l}\text { Rigid spineboard, } \\
\text { soft-layered spineboard }\end{array}$ \\
\hline Number & 15 & 15 & 30 & - \\
Gender (M/F) & $11 / 4$ & $11 / 1$ & $19 / 11$ & $\mathrm{~b}$ \\
Age (years) & $51.5(16.2)$ & $49.6(18.9)$ & $39.0(11.1)$ & a,b \\
BMI (kg/m ${ }^{2}$ ) & $24.5(2.9)$ & $24.2(3.2)$ & $24.7(3.4)$ & n.s. \\
Type of surgery & & & & n.s. \\
Hernia inguinalis & $10 / 15$ & $11 / 15$ & n.a. & n.a. \\
Hernia epigastrica & $3 / 15$ & - & n.a. \\
Hernia umbilicalis & $2 / 15$ & $2 / 15$ & n.a. \\
Hernia inguinoscrotalis & - & $2 / 15$ & n.a. \\
$\begin{array}{l}\text { Time on immobilisation } \\
\text { device (minutes) }\end{array}$ & $63(18)$ & $65(12)$ & & n.s. \\
n.a.: not applicable & & &
\end{tabular}

\section{Tissue-interface pressures}

In both awake volunteers and anaesthetised patients, peak pressures for scapula and sacrum and peak pressure index (PPI) at the sacrum were significantly lower on the soft-layered spineboard than on the rigid spineboard (Figures 3.1a-d). Peak pressures at the heel were similar for both devices for awake volunteers, and were significantly lower for patients under anaesthesia on the soft-layered spineboard than for the healthy volunteers. Peak pressures and sacrum PPI on the soft-layered spineboard were significantly lower for anaesthetised patients compared to awake volunteers $(P<$ 0.01). Peak pressures on rigid spineboard did not differ between anaesthetised 
patients and awake volunteers $(P>0.05)$. No significant changes in pressure were seen during the course of the pressure measurements $(P>0.05)$.
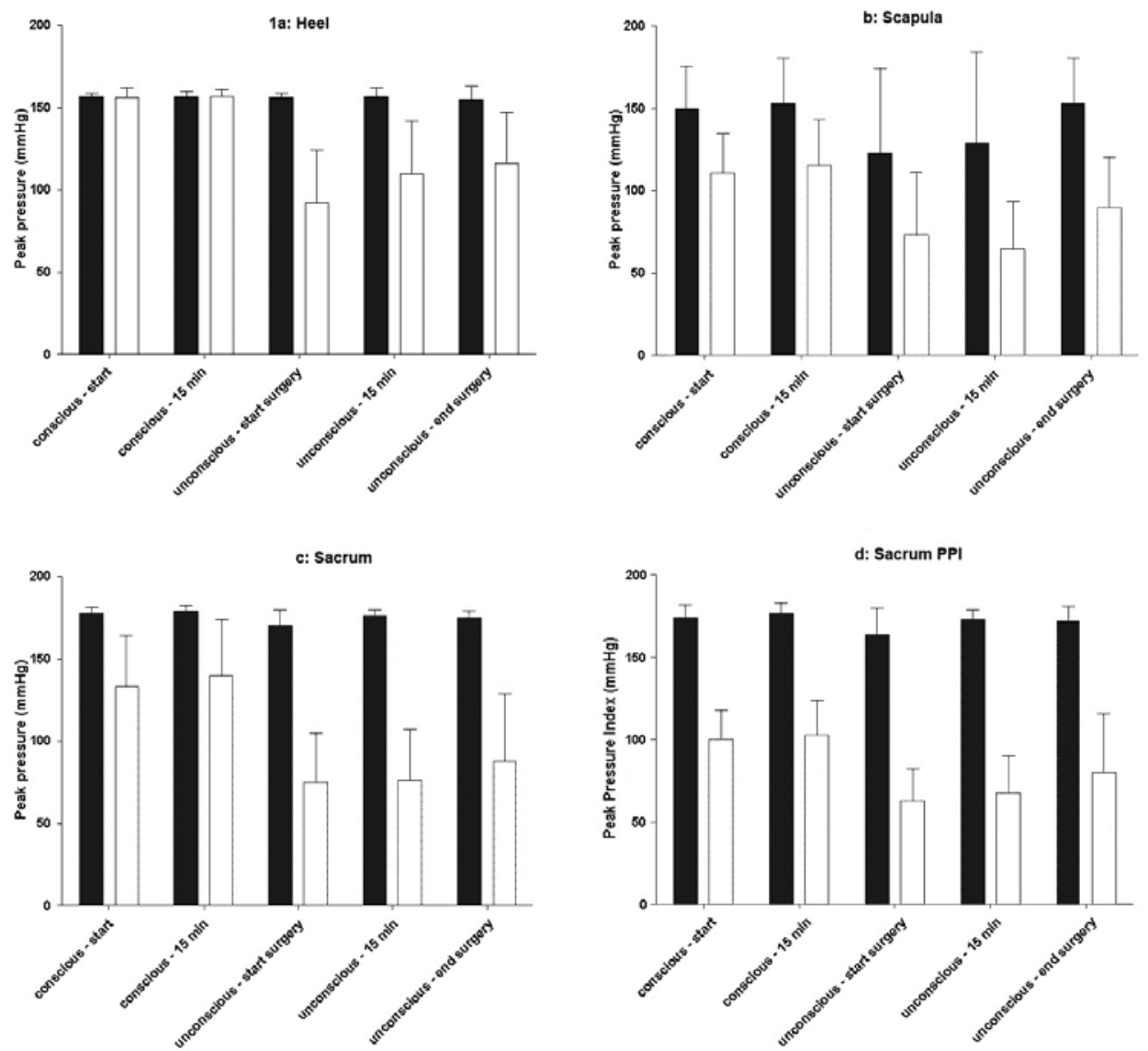

Figure 3.1a-d. Tissue-interface pressures at various parts of the body; mean peak pressures and standard deviation for rigid spineboard (dark grey bars) and soft-layered spineboard (white bars). a. Tissue-interface pressures on the heel. b. Tissue-interface pressures on the scapula. c. Tissue-interface pressure on the sacrum. d. Tissue-interface peak-pressure index on the sacrum.

\section{Redness}

Redness was assessed for anaesthetised patients only (Figures 3.2 and 3.3). There was a clear difference in redness on the sacrum after surgery, with significantly more patients without any redness at all on the soft-layered spineboard and significantly more volunteers with clearly defined redness on the rigid spineboard (Table 3.2). In all cases the redness was blanchable and had disappeared at the 2 hour postoperative follow up assessment. 


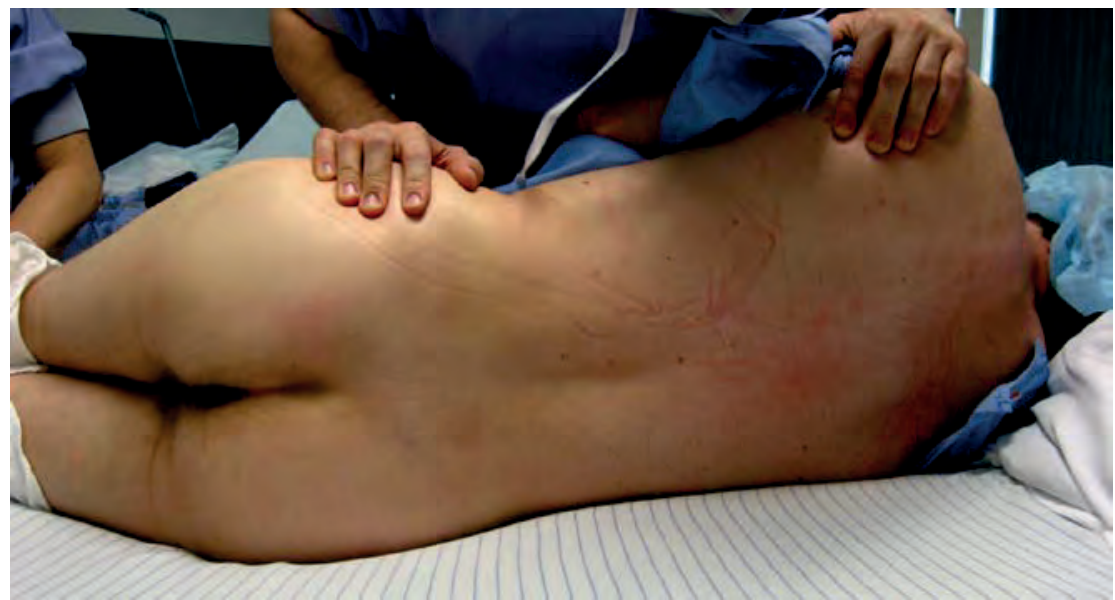

Figure 3.2. Example of diffuse redness on the sacrum after lying on the soft-layered spineboard during surgery.

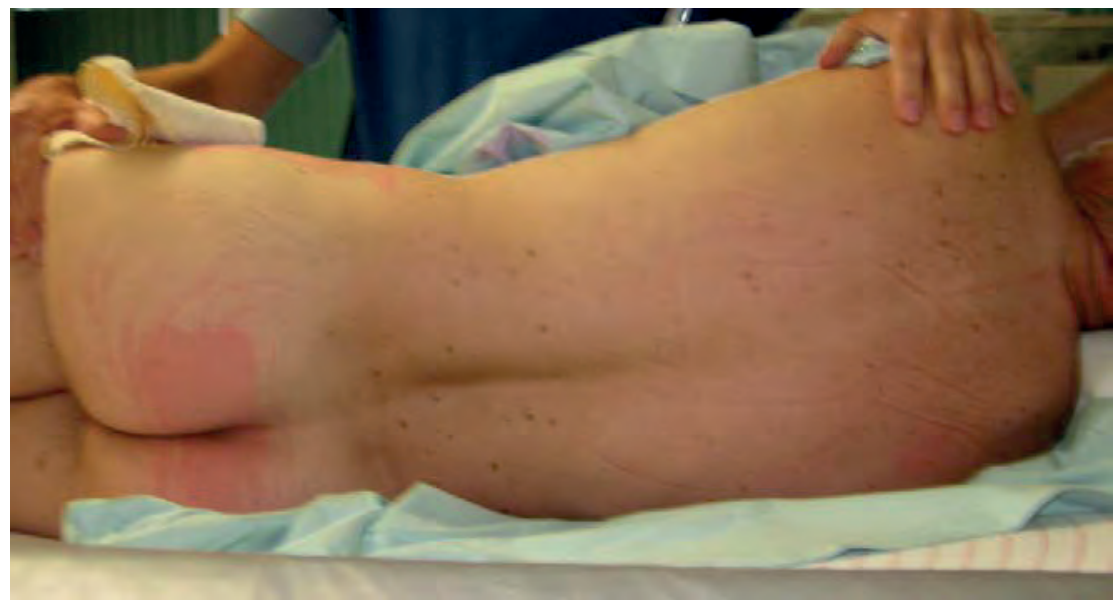

Figure 3.3. Example of clearly defined redness on the sacrum after lying on the rigid spineboard during surgery.

Table 3.2. Redness at the sacrum, anaesthetised patients*.

\begin{tabular}{llll}
\hline Redness at the sacrum & Rigid spineboard & Soft-layered spineboard & Total \\
\hline No redness & 1 & 9 & 10 \\
Diffuse redness & 5 & 6 & 11 \\
Clearly defined redness & 9 & 0 & 9 \\
Total & 15 & 15 & 30 \\
\hline
\end{tabular}

${ }^{*} \chi 2=15.49, P<0.01$ for rigid spineboard vs soft-layered spineboard 


\section{Discussion}

This study showed that unconscious subjects placed on a soft-layered spine board had lower tissue-interface pressures than conscious subjects, while no differences between these two groups were found when using a rigid spine board. Patients under anaesthesia and awake controls both had significantly lower tissue-interface pressures on the soft-layered spineboard than on the rigid spineboard.

Despite a growing body of evidence that immobilising patients on a rigid spineboard can cause a number of adverse effects such as aspiration, respiratory problems, pain, and pressure ulceration [6,10,20-25], the use of spineboards is expected to continue in practice. In addition to efforts to increase awareness in order to limit the use of spineboards, more tissue-protective devices for spinal immobilisation have been developed to minimise the risks of pain and pressure ulcer development. However, evidence showing how these products compare with traditionally used rigid spineboards is scarce. In an earlier study [10] we compared the tissue-interface pressures between the rigid spineboard and the soft-layered spineboard in healthy, awake volunteers. It was unknown, however, whether these results for conscious healthy subjects could be translated to the target group of patients incapable of moving to relieve pressure, such as unconscious patients or patients with spinal cord injury. In this study we therefore looked at anaesthetised patients as a proxy for these patient categories. The average duration of the surgical procedure was about one hour, which is in practice equal to the average time a trauma patient is immobilised on a spineboard $[2,3]$.

Earlier studies showed that the use of a rigid spineboard leads to discomfort and high tissue-interface pressures $[6,9,10,16,21]$. We have also previously demonstrated that using a rigid spineboard led to a considerably higher maximum tissue strain than the threshold at which deformation damage can occur [14]. These high tissue-interface pressures are therefore a significant risk factor for the development of pressure ulcers [26], which are both debilitating for the patient [27-29] and costly for the hospital $[4,8,30]$. We found equally high tissue-interface pressures for both awake volunteers and anaesthetised patients using the rigid spineboard, with values comparable to those from earlier studies $[6,10,16]$. The tissue-interface pressures experienced by the unconscious patients on the rigid spineboard remained comparable to those for the awake subjects. In addition, tissue-interface pressures on the soft-layered spineboard were significantly lower for the unconscious patients than for the awake volunteers. Both these observations are remarkable, as they did not confirm our initial hypothesis that both spineboards would lead to an increase in pressures. The data from our study suggest that each board has a ceiling effect. The rigid spineboard reaches this ceiling almost immediately, while the tendency of the pressure to increase on the soft-layered 
spineboard suggests that its ceiling effect is reached later. Although the former may be speculative, the bottom line remains that the risk of tissue damage by pressureinduced tissue deformation is reduced by using the soft-layered spineboard compared to the rigid spineboard.

After removal from the spineboard, reactive hyperaemia occurred, indicating that there had been a period of relative hypoperfusion. This was especially noticeable with the rigid spineboard, with 9 out of 15 patients showing clearly defined redness, compared to 0 out of 15 patients on the soft-layered spineboard. There is a trade-off between pressure and time regarding pressure-induced tissue damage with higher pressures causing tissue damage sooner than lower pressures [31,32]. In our study the pressures did not lead to permanent damage to the soft tissue; the redness had disappeared in all patients at the follow up assessment of the skin two hours after the end of the surgery. The patients remained haemodynamically stable during the procedure and had only low-risk comorbidities. Tissue hypoperfusion leading to tissue ischaemia and cell necrosis occurs more rapidly in hypotensive patients because of compromised microcirculation caused by poor perfusion [25]. Especially patients with shock or spinal cord damage combined with altered conscious levels are at increased risk of hypotension, so immobility, time or pressure related risk factors should be reduced.

A number of comments need to be made on the interpretation of our observations. First, although weight and body mass index of the participants were representative of those in the general Dutch population, age and gender were not completely comparable between healthy volunteers and the patients undergoing surgery and anaesthesia. Second, our study was single-blinded, as the researcher who performed the pressure measurements and redness evaluations knew which device the volunteer was lying on. However, pressure measurements were objectified by using the Xsensor pressure mapping device. Redness was objectified by testing blanchableness using a clear plastic disc. Photos were taken to enable classification of the redness afterwards. The redness of the skin was also judged by an independent, blinded observer with no knowledge of the allocation of the patients. Kappa was 0.811 and differences of opinion were resolved through discussion. Furthermore, visual inspection of the skin was undertaken as an indicator of tissue damage. Although hyperaemia was noted in a significant number of patients, we do not know how this relates to tissue ischaemia and cell necrosis. Further studies should therefore focus on the relation between local tissue-interface pressures and indicators of tissue damage.

In conclusion, this randomised study found tissue-interface pressures to be significantly lower for the soft-layered spineboard than for the rigid spineboard, for both awake volunteers and anaesthetised patients. Furthermore, tissue-interface pressures using the soft-layered spineboard were lower for the anaesthetised patients 
than for the awake volunteers. Redness of the skin of the sacrum was significantly less on the soft-layered spineboard than on the rigid spineboard. These findings show the importance of using a soft-layered spineboard for tissue-interface pressure reduction, and may be of importance for trauma patients with a reduced level of consciousness. 


\section{References}

1. American College of Surgeons Committee on Trauma. Advanced Trauma Life Support for Doctors. 8th ed. Chicago, IL: American College of Surgeons; 2008.

2. Cooney DR, Wallus $\mathrm{H}$, Asaly M \& Wojcik S. Backboard time for patients receiving spinal immobilization by emergency medical services. Int J Emerg Med 2013;6:17-9.

3. Lerner EB \& Moscati R. Duration of patient immobilization in the ED. Am J Emerg Med. 2000;18:28-30.

4. Allman RM, Goode PS, Burst N, Bartolucci AA \& Thomas D. Pressure ulcers, hospital complications, and disease severity: impact on hospital costs and length of stay. Adv Skin Wound Care. 1999;12:2230.

5. Baldwin KM \& Ziegler SM. Pressure ulcer risk following critical traumatic injury. Adv Skin Wound Care. 1998;11:168-75.

6. Cordell WH, Hollingsworth JC, Olinger ML, Stroman SJ \& Nelson DR. Pain and tissue-interface pressure during spine-board immobilization. Ann Emerg Med. 1995;26:31-6.

7. Dealey C, Posnett J \& Walker A. The cost of pressure ulcers in the United Kingdom. J Wound Care. 2012;21:261-6.

8. Graves N, Birrell FA \& Whitby M. Modeling the economic losses from pressure ulcers among hospitalized patients in Australia. Wound Repair Regen. 2005;13:462-7.

9. Hauswald M, Hsu M \& Stockoff C. Maximizing comfort and minimizing ischemia: a comparison of four methods of spinal immobilization. Prehosp Emerg Care. 2000;4:250-2.

10. Hemmes B, Poeze M \& Brink PRG. Reduced tissue-interface pressure and increased comfort on a newly developed soft-layered long spineboard. J Trauma. 2010;68:593-8.

11. Watts D, Abrahams E, MacMillan C, et al. Insult after injury: pressure ulcers in trauma patients. Orthop Nurs. 1998;17:84-91.

12. Zlupko GR, Megargel RE \& O'Connor RE. Patient discomfort due to indiscriminant use of spinal immobilization. Prehosp Emerg Care. 2004;8:101.

13. Dealey C \& Lindholm C. Pressure ulcer classification. In: Romanelli M, Clarck M, Cherry G, Colin D, Defloor T, editors. Science and practice of pressure ulcer management. London, UK: Springer London; 2006.

14. Oomens CWJ, Zenhorst W, Broek M, et al. A numerical study to analyse the risk for pressure ulcer development on a spine board. Clin Biomech. 2013;28:736-42.

15. Vanderwee K, Grypdonck MHF, de Bacquer D \& Defloor T. The reliability of two observation methods of nonblanchable erythema, grade1 pressure ulcer. Appl Nurs Res. 2006;19:156-62.

16. Keller BPJA, van Overbeeke J \& van der Werken Chr. Interface pressure measurement during surgery: a comparison of four operating Table surfaces. J Wound Care. 2005;15:5-9.

17. Amlung SR, Miller WL \& Bosley LM. The 1999 National Pressure Ulcer Prevalence Study: a benchmarking approach. Adv Skin Wound Care. 2001;14:297-301.

18. Vanderwee KM, Clark M, Dealey C, Gunningberg L \& DeFloor T. Pressure ulcer prevalence in Europe: a pilot study. J Eval Clin Pract. 2007;13:227-35.

19. Vangilder C, Macfarlane GD \& Meyer S. Results of nine international pressure ulcer prevalence surveys: 1989 to 2005. Ostomy Wound Manage. 2008;54:40-54.

20. Brownlee AE. Managing spinal immobilisation devices in the emergency department. Australas Emerg Nurse J. 2005;8:79-83.

21. Chan D, Goldberg RM, Mason J \& Chan L. Backboard versus mattress splint immobilization: a comparison of symptoms generated. J Emerg Med. 1996;14:293-8.

22. Kwan I \& Bunn F. Effects of prehospital spinal immobilization: a systematic review of randomized trials on healthy subject. Prehospital Disaster Med. 2005;20:47-53. 
23. Vickery D. The use of the spinal board after the pre-hospital phase of trauma management. Emerg Med J. 2001;18:51-4.

24. Bauer D \& Kowalski R. Effect of spinal immobilization devices on pulmonary function in the healthy, nonsmoking man. Ann Emerg Med. 1988;17:915-8.

25. Berg G, Nyberg S, Harrison P, Baumchen J, Gurss E \& Hennes E. Near-infrared spectroscopy measurement of sacral tissue oxygen saturation in healthy volunteers immobilized on rigid spineboards. Prehosp Emerg Care. 2010;14:419-24.

26. DeFloor T. The risk of pressure sores: a conceptual scheme. J Clin Nurs. 1999;8:206-16.

27. Hopkins A, Dealey C, Bale S, DeFloor T \& Worboys F. Patient stories of living with a pressure ulcer. J Adv Nurs. 2006;56:345-53.

28. Gorecki C, Brown JM, Nelson EA, et al. Impact of pressure ulcers on quality of life in older patients: a systematic review. J Am Geriatr Soc. 2009;57:1175-83.

29. Spilsbury K, Nelson A, Cullum N, Iglesias C, Nixon J \& Mason S. Pressure ulcers and their treatment and effects on quality of life: hospital inpatient perspectives. J Adv Nurs. 2007;57:494-504.

30. Bennett GG, Dealey C \& Posnett J. The cost of pressure ulcers in the UK. Age Ageing. 2004;33:230-5.

31. Reswick J \& Rogers J. Experience at the Rancho Los Amigos Hospital with devices and techniques to prevent pressure sores. In: Reswick J, Rogers J, editors. Bed sore mechanics. London: MacMillan Press; 1976. p. 301-10.

32. Linder-Ganz E, Engelberg S, Scheinowitz M \& Gefen A. Pressure-time cell death threshold for albino rat skeletal muscles as related to pressure sore biomechanics. J Biomech. 2006;39:2725-32. 



\section{A numerical study of the risk for pressure ulcer development on a spineboard}
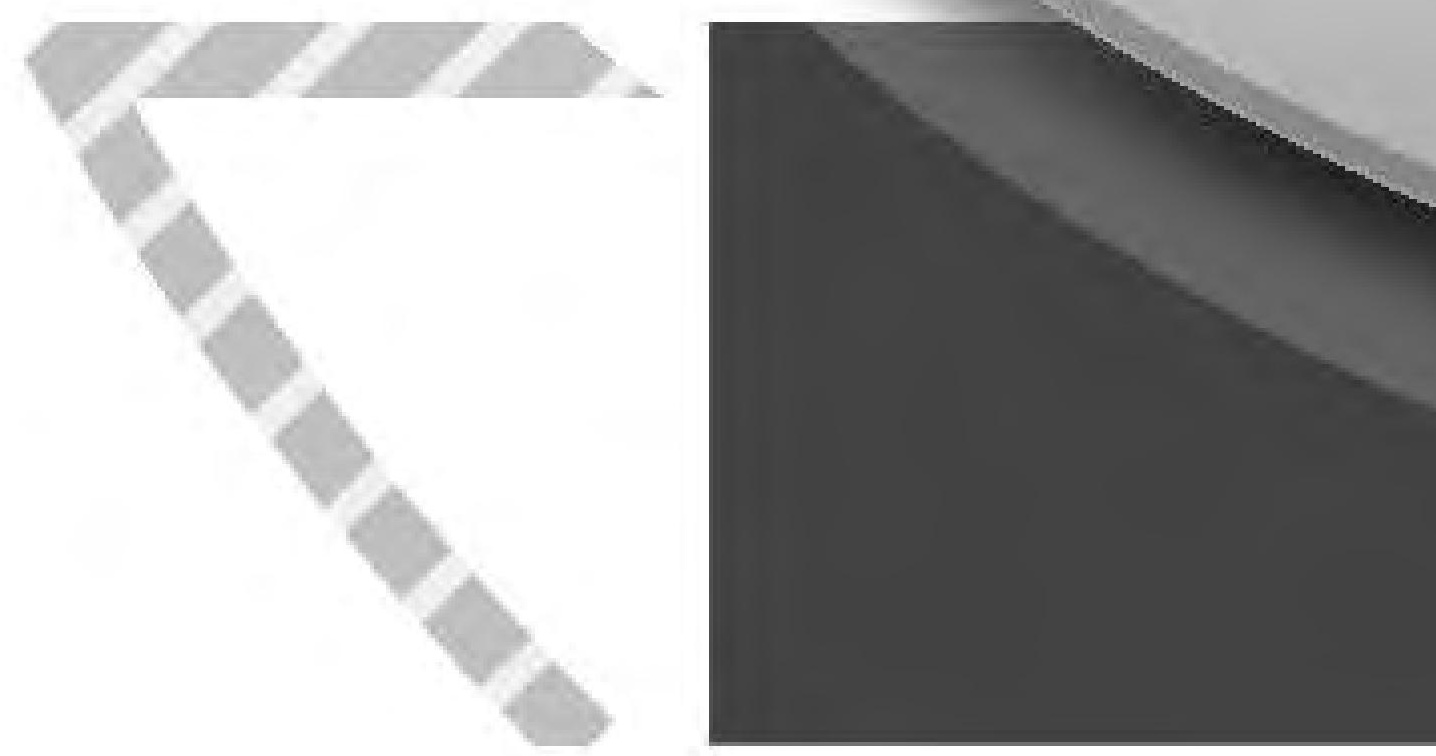

Cees W.J. Oomens, Willem Zenhorst, Merel Broek, Baukje Hemmes, Martijn Poeze, Peter R.G. Brink, Dan L. Bader

Clinical Biomechanics. 2013;28: 736-742 


\section{Abstract}

Background: Spineboards are used to immobilise accident victims suspected of having spinal injury. Guidelines for the maximum time patients should remain on the board are often exceeded and this may occasionally lead to pressure ulcers. Aetiological research has shown that two processes ultimately lead to pressure ulcers: "Ischaemic damage", which takes several hours to develop, and "deformation damage" at high strains. The latter process is very rapid, and the first signs of cell damage are already evident within minutes. In order to minimise the risk of pressure ulcer development during prolonged loading, a new soft-layered spineboard has been designed.

Methods: A subject-specific numerical approach was adopted to evaluate the prototype spineboard in comparison to a conventional spineboard, in terms of the estimated strains in the soft tissues adjacent to the sacrum in the supine position. The geometry of the model was derived from magnetic resonance images of three human volunteers in an unloaded situation. The images of the loaded situation were used to "tune" the material parameters of skin, fat and muscle. The prediction of the deformed contours on the soft-layered board was used to validate the model.

Results: Comparison of the internal strains in muscle tissue near the spine showed that these were reduced on the soft-layered board, and that maximum strains were considerably lower than the threshold at which deformation damage is possible. By contrast, this threshold was exceeded in all cases on the rigid spineboard.

Conclusions: The prototype soft-layered spineboard is able to reduce the risk for deformation damage and thus reduces the risk of developing pressure ulcers. 


\section{Introduction}

The spineboard is a device used for spinal immobilisation of accident victims suspected of having a spinal injury. This method of immobilisation is well established in prehospital and in-hospital trauma protocols [1, 2]. Although existing guidelines recommend minimising the time spent on the spineboard $[3,4]$, in practice, patients remain on the board for prolonged periods, due to on-scene treatment and evaluation in the emergency room and/or the radiological facility $[5,6]$. Since the spine needs to be immobilised during this period, removing patients from the spineboard is a low priority issue for the emergency room staff. Indeed, the average time patients spend on a spineboard has been estimated at 77 minutes $[7,8]$, while in some cases it can exceed 2 hours $[9,10]$. Although stabilisation of the spine remains a critical requirement for trauma patients, it is also clear that prolonged immobilisation on the spineboard causes pain and discomfort [11-13] and may occasionally lead to the development of pressure ulcers [11, 14, 15]. Devices for pre-hospital care can be divided into two groups: those with a rigid surface and those with a softer padded surface. All currently available spineboards have a rigid surface. Some manufacturers have developed optional padding to soften the surface of a rigid spineboard. The vacuum mattress, when deflated, also has a rigid surface. The soft-layered spineboard is the only device with a built-in soft surface.

"A pressure ulcer (PU) is a localised injury to the skin and/or underlying tissue, usually over a bony prominence, as a result of pressure or pressure in combination with shear" [16]. In addition to individual suffering, the development of PUs leads to prolonged hospital stays [17, 18] and increased treatment costs [19]. The incidence of PUs in European hospitals is estimated to be between 18 and 20\% [20], and in the 10 to $18 \%$ range in USA hospitals [21]. In the Netherlands, the incidence rate in hospitals is estimated to be between 5 and $8 \%$ (excluding category 1 PUs), and this value will inevitably be higher in residential care and community settings [22].

In the last decade, considerable progress has been made in understanding the aetiology of PUs. There is increasing consensus that, although the majority of pressure ulcers start at the skin surface and may extend to deeper layers, there is also a category that starts in deeper soft tissue layers adjacent to bony prominences. In the 2009 European guidelines for PU prevention and treatment [16] the latter are referred to as deep tissue injury (DTI). DTI is especially an issue for patients who have impaired sensation, most notably spinal cord injured individuals, or unconscious patients. In this case the subject does not feel the mechanically induced subcutaneous damage, and it is not uncommon that these wounds are only identified after they have become very severe. It is hypothesised that superficial wounds mostly result from a combination of shear forces and pressures at the interface between skin and supporting surfaces, 
while DTI is a result of high stresses and strains adjacent to bone. We propose that spineboards could lead to DTI. Vulnerable areas for DTI while lying the spineboard are the sacrum, the heels, the shoulders and the back of the head.

In the last 15 years a variety of model systems have been used to study the aetiology of DTI, ranging from in vitro studies on single cells $[23,24]$ to tissueengineered muscle constructs [25-27] and animal studies [28-31]. All these studies, which focused on skeletal muscle cells and tissues, confirmed that two damage mechanisms play a major role in prolonged mechanically induced damage. In most practical situations it is impossible to prevent the occlusion of blood vessels, resulting in partial or complete reduction of blood perfusion of mechanically loaded body sites. This leads to a state of ischaemia in the tissue, resulting in hypoxia. Accordingly, muscle tissue changes from an aerobic to an anaerobic metabolism. This causes accumulation of metabolic waste products in the interstitial spaces, lowering of the $\mathrm{pH}$ and eventually cell death. Healthy subjects will normally use pressure relieving strategies, involving changing body position, to minimise the risk of damage accumulation.

The second damage mechanism involves direct deformation damage at a threshold level, which for maximum shear strain has been estimated to be between 0.5 and 0.6 (50 to 60\%) [30]. The cause of this mechanism is not yet clear. It has been proposed that it is a matter of failure of the cell membrane or direct disruption of the cytoskeletal elements. Nonetheless, all model systems revealed a strong correlation between local tissue strain and damage development [30]. It is also evident from studies with human volunteers that internal tissue strains adjacent to bony prominences often exceed the strain threshold level during prolonged sitting [32].

The current study estimated the mechanical strains in sacral tissues of subjects supported on a spineboard. Two different support surfaces were considered, a standard rigid spineboard and a prototype spineboard with a soft-covered inlay, referred to as soft-layered spineboard [33]. A recent comparative study reported increased comfort and significantly lower interface pressures on the soft-layered spineboard [33].

Two specific questions were addressed. (1) Is the deformation threshold exceeded in the sacral area of subjects lying supine on a spineboard? (2) Is the prototype softlayered spineboard capable of reducing the internal strains to values below the deformation damage threshold? We attempted to answer these questions using a combination of magnetic resonance imaging (MRI) and finite element (FE) modelling. 


\section{Methods}

MR images of the sacral area of able-bodied volunteers were recorded on both support surfaces. These images were used to develop a subject-specific FE model, which was tuned and validated on the images of the undeformed and deformed condition on the standard spineboard and used to calculate internal tissue strains in the soft tissues near the sacrum.

\section{Image acquisition}

The geometry of the model was based on MR images of 13 volunteers, with varying morphology, each scanned lying supine on both the rigid spineboard and the softlayered spineboard. Images were also recorded with the subject in prone position in a standard MRI to depict the unloaded shape of the sacral region. Approval was obtained from the Medical Ethics Committee of the Maastricht University Medical Centre. Written informed consent was obtained from all volunteers before they were enrolled in the study.

After extensive inspection of all data sets, three representative subjects were selected to generate three anatomically accurate FE meshes. The selection was based on obtaining sufficient variety of anatomy and body mass index, visual accuracy of the data sets and whether the subject's position had been correctly aligned to the MRI scanner. The characteristics of the three subjects are summarised in Table 4.1. The images obtained in prone position serve as an unloaded reference state of the buttocks area, on which the FE mesh was based. The images obtained on the rigid spine board and the soft-layered spineboard were compared with the output of the model. The specific focus of the model involved the most outward pointing prominences of the sacrum (the medial sacral crest, a point located at a few centimetres above the coccyx) and the femoral heads, as indicated in Figure 4.1.

Table 4.1. Subject characteristics

\begin{tabular}{llllll}
\hline Subject & BMI $(\mathbf{k g} / \mathbf{m} 2)$ & Weight $(\mathbf{k g})$ & Height $(\mathbf{m})$ & Age (years) & Gender \\
\hline I & 19.4 & 49 & 1.59 & 27 & Female \\
II (reference) & 24.0 & 68 & 1.72 & 29 & Female \\
III & 27.4 & 77 & 1.68 & 41 & Female \\
\hline
\end{tabular}



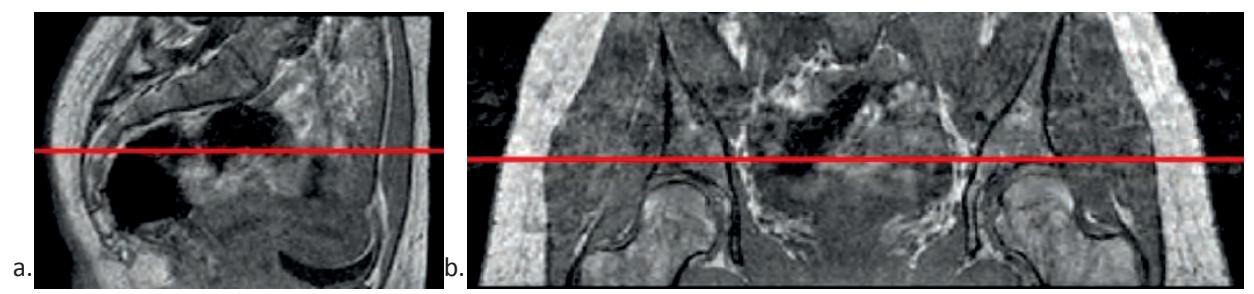

Figure 4.1a-b. Area of interest. a. Side view of the hip area. The red line a few centimeters above the coccyx is the region of interest. $\mathbf{b}$. Front view of the hip area. The red line a few centimeters above the femur heads is the region of interest.

\section{Mesh development}

A subject-specific two-dimensional, plane-stress FE model was developed of the selected subjects, based on the high resolution images obtained close to the sacral area. The images obtained in prone position were used for the segmentation and considered to represent the undeformed state of the tissue area (Figure 4.2a). Images were segmented in the Mimics software code (Materialise NV, Leuven, Belgium). After segmentation a mesh was developed with quadratic triangles, and transferred to Marc Mentat 2008r1 (MSC Software).

a.
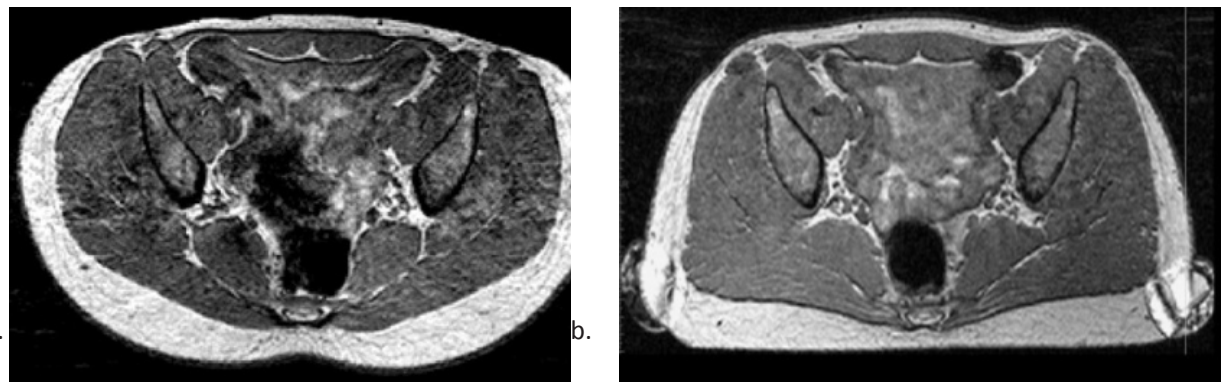

Figure 4.2a-b. High resolution MRI images of the sacral area. a.MRI image in prone position (image is rotated $180^{\circ}$ for easy comparison with the supine image). b. MRI image in supine position on the rigid spineboard.

\section{Material properties}

The model had to represent four components: bone, muscle, fat and skin tissues. The region in the mesh ascribed to bone was in fact tissue consisting of very stiff ligaments (Table 4.2), but we assumed this would not affect the strains in the area of interest around the sacrum. The skin, fat and muscle tissues were modelled with an incompressible, nonlinear Ogden material law with strain energy density W (34):

$$
W=\frac{\mu}{\alpha}\left(\lambda_{1}^{\alpha}+\lambda_{1}^{\alpha}-\lambda_{3}^{\alpha}-3\right)
$$

with $\lambda 1,2,3$ the principal stretch ratios and $\mu[\mathrm{kPa}]$ and $\alpha[-]$ the material properties. 
Table 4.2 Material properties of the various tissues in the FE model after tuning the model on the images in supine position.

\begin{tabular}{lll}
\hline Tissue & $\mathbf{M}[\mathrm{kPa}]$ & $\mathbf{A}[-]$ \\
\hline Skin & 8 & 5 \\
Fat & 1 & 5 \\
Muscle & 0.3 & 5 \\
& Young's modulus $E(\mathrm{kPa})$ & Poisson's ratio \\
Bone & $3.4 * 106$ & 0.36 \\
\hline
\end{tabular}

A series of parameter studies with the reference model (model II in Table 4.1) were performed to test the validity of the FE model. In particular, the measurable contours of the image in the deformed condition on the rigid spineboard were compared with the FE predictions. First the mesh density was varied to find the optimal compromise between computer time and accuracy. Close examination of the 2-D model as a representation of a 3-D subject revealed that a plane-stress analysis, as opposed to a plane-strain analysis, yielded the best fit on the MRI images. This suggests that tissues can deform easily in the direction perpendicular to the plane of the view. The friction coefficient between skin and supporting surface was varied, and although this only marginally influenced the contours, it did have a large influence on the calculated shear strains in the skin. Accordingly, a worst-case scenario was selected with a friction coefficient of 1 . The fitting was mostly influenced by the choice of the material parameters assigned to skin, fat and muscle. The image revealed a clear distinction between the tissue layers, so each tissue could be manually fitted, leading to the material properties detailed in Table 4.2. The bone and stiff ligaments were modelled as an isotropic, linear elastic material with a high modulus of elasticity (3.4 [GPa]). This resulted in the mesh and material groups shown in Figure 4.3.

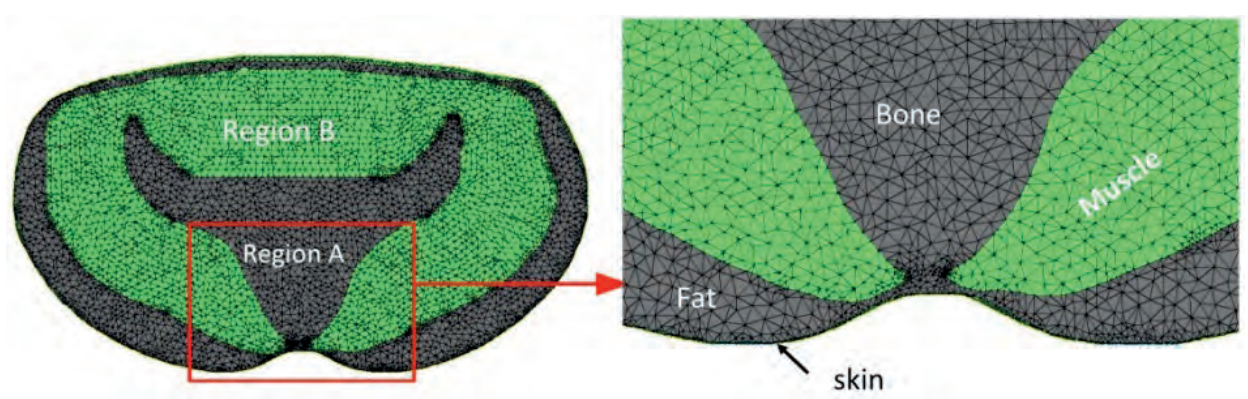

Figure 4.3. The FE mesh for the reference model, with the different materials groups used.

\section{Boundary conditions}

A contact option between skin and supporting surface was used with Coulomb friction and a friction coefficient of 1 . First, the support was moved upwards until contact was established between the skin and the surface. During this phase, a central node in the 
model was fixed to prevent rigid-body motion of the body. Secondly, a body force was applied to the body to simulate gravity. This body force was based on the density of water $(10-5 \mathrm{~N} / \mathrm{mm} 3)$. By integrating over the total area of the mesh, and taking the product of slice thickness $(1 \mathrm{~mm})$ the body force was assumed to approach the weight of the modelled slice at the sacral region of the adult human model.

The conventional rigid spineboard was modelled as a rigid surface. For the softlayered spineboard an FE model was built that included the inlay and the cover layer which makes up its inlay (Figure 4.4). Indentation tests were performed to measure the material properties of the inlay. The very large deformations observed in the experiments on the soft-layered spineboard (up to 90\%) led to convergence problems when using solid elements. Accordingly, the stiffness of the entire structure was represented by line elements acting as nonlinear, extensional springs that were fixed to a "virtual" line a few centimetres above the inlay. Again parameters were tuned in such a way that the outer contour in the loaded condition in the reference model matched the measured contours. Identical properties were used for the simulations in models I and II.

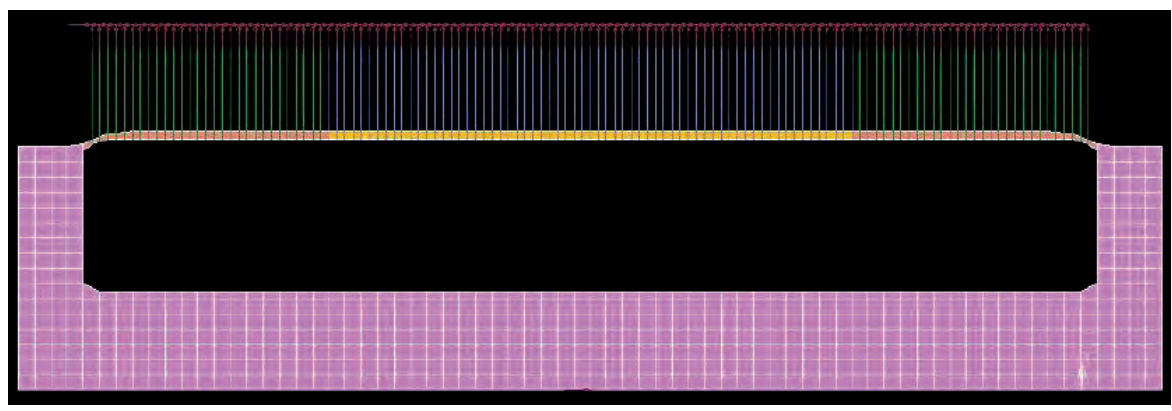

Figure 4.4. FE mesh of the soft-layered long spineboard. The rigid board is shown in pink. The springs suspending the top layer of the inlay are shown in green. The top nodes of the springs are fixed in space along a virtual line.

\section{Results}

Figure 4.5 shows the images of the deformed condition and the numerically determined contours of the model projected onto the images of the 3 subjects on the rigid spineboard. The model was "tuned" on the reference subject, with a focus on the area of interest, as indicated by the yellow dotted box in Figure 4.6b. The same material parameter set was used for all three subjects, so the only difference between model I (Figure 4.5a) and model III (Figure 4.5c) is the subject-specific geometry of the bones and tissues. The model matches the images of the three subjects reasonably 
accurately, suggesting minor differences in the material properties of their individual tissues.
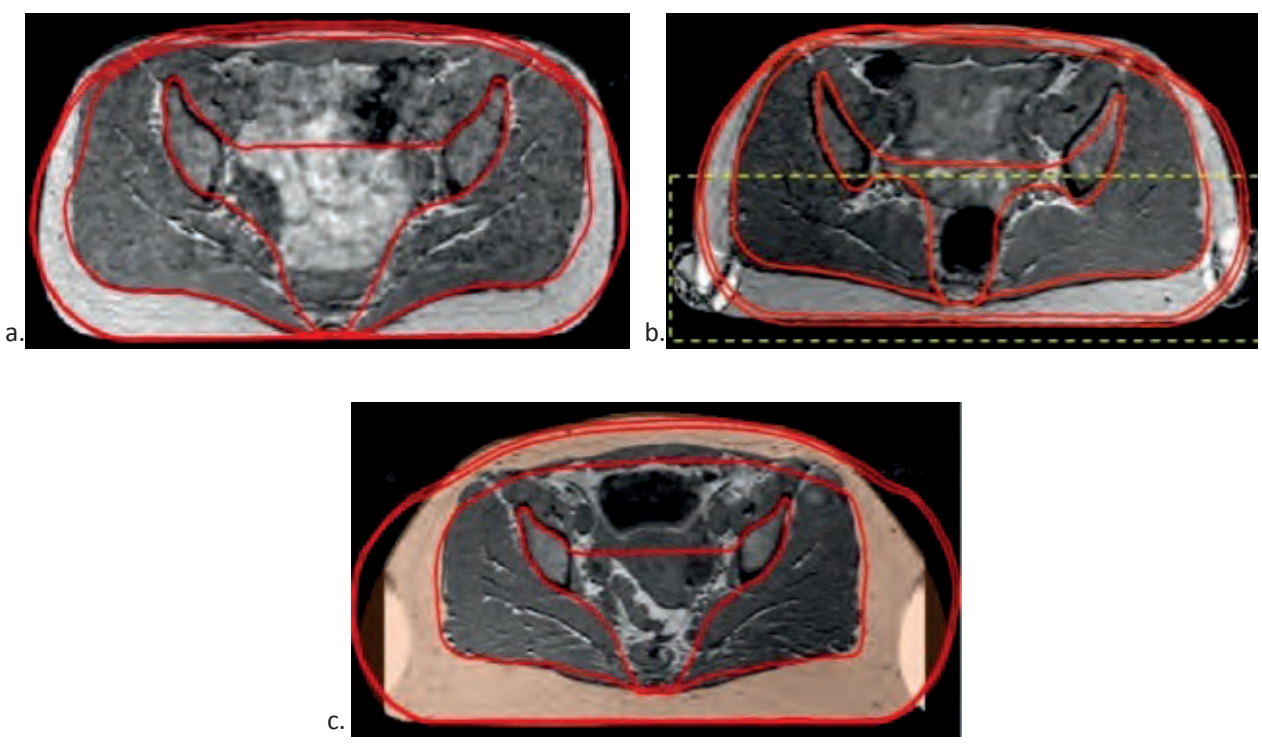

Figure 4.5a-c. MR images of the three subjects on the rigid spineboard, with the contours of the deformed mesh in red. a. Subject I; b. Subject II (reference model); c. Subject III.

Figures 4.6a-c show the corresponding images and contours for the same subjects lying on the soft-layered spineboard. It is interesting to note that the reference model now provides the lowest accuracy, with a clear difference between the contours surrounding the fat layer and the MR images. By contrast, the contours derived from models I and III present a very good match with the images. The estimated distribution of the maximum shear strains calculated with the reference model for both the spineboard and the soft-layered spineboard reveals high strains, in excess of $50 \%$, in the muscle and the fat around the sacrum on the rigid spineboard, whereas such high strains are absent in the corresponding layers for the soft-layered spineboard (Figure 4.7). High strains were also found in the area around the ilium bones. These finding are not, however, considered to represent a realistic situation, as the tissues around these bones were modelled with the same properties as muscle, and the tissues cannot slide over the bone. 

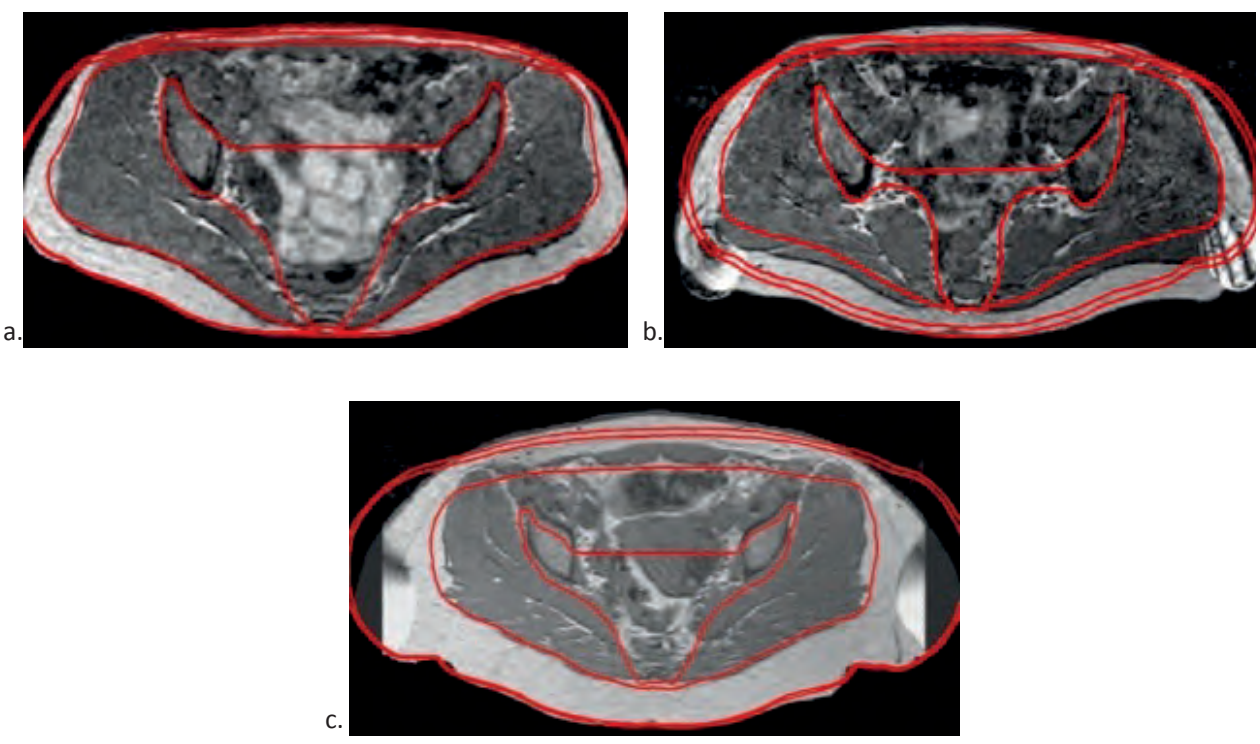

Figure 4.6a-c. MR images of the three subjects on the soft-layered spineboard, with the contours of the deformed mesh in red. a. Subject I; b. Subject II (reference model); c. Subject III.

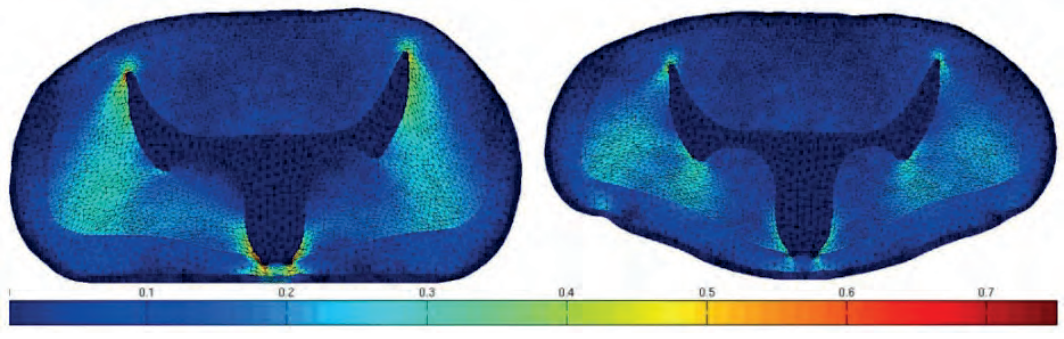

Figure 4.7. Distribution of maximum shear strains in the deformed mesh of the reference subject (II), on the rigid spineboard (left) and on the soft-layered spineboard (right)

A more quantitative comparison between the three subjects and between the spineboard and the soft-layered spineboard was achieved by selecting a region of interest (ROI) around the sacrum, depicted as region $A$ in Figure 4.3. Within the rectangular $\mathrm{ROI}$, the relative area corresponding to different strain ranges was plotted for all cases. Figure 4.8a depicts the histogram for the area around the sacrum for maximum shear strains ranging from 0 to 0.8 . The shaded area in the range between 0.5 and 0.6 corresponds to the threshold range where damage due to deformation has been shown to be initiated [30,35]. Figure 4.8b magnifies the estimated strains between 0.3 and 0.8 . It is clear that for all subjects the rigid spineboard yields strains 
within the critical strain range, while on the soft-layered spineboard all maximum shear strains are less than 0.5 .
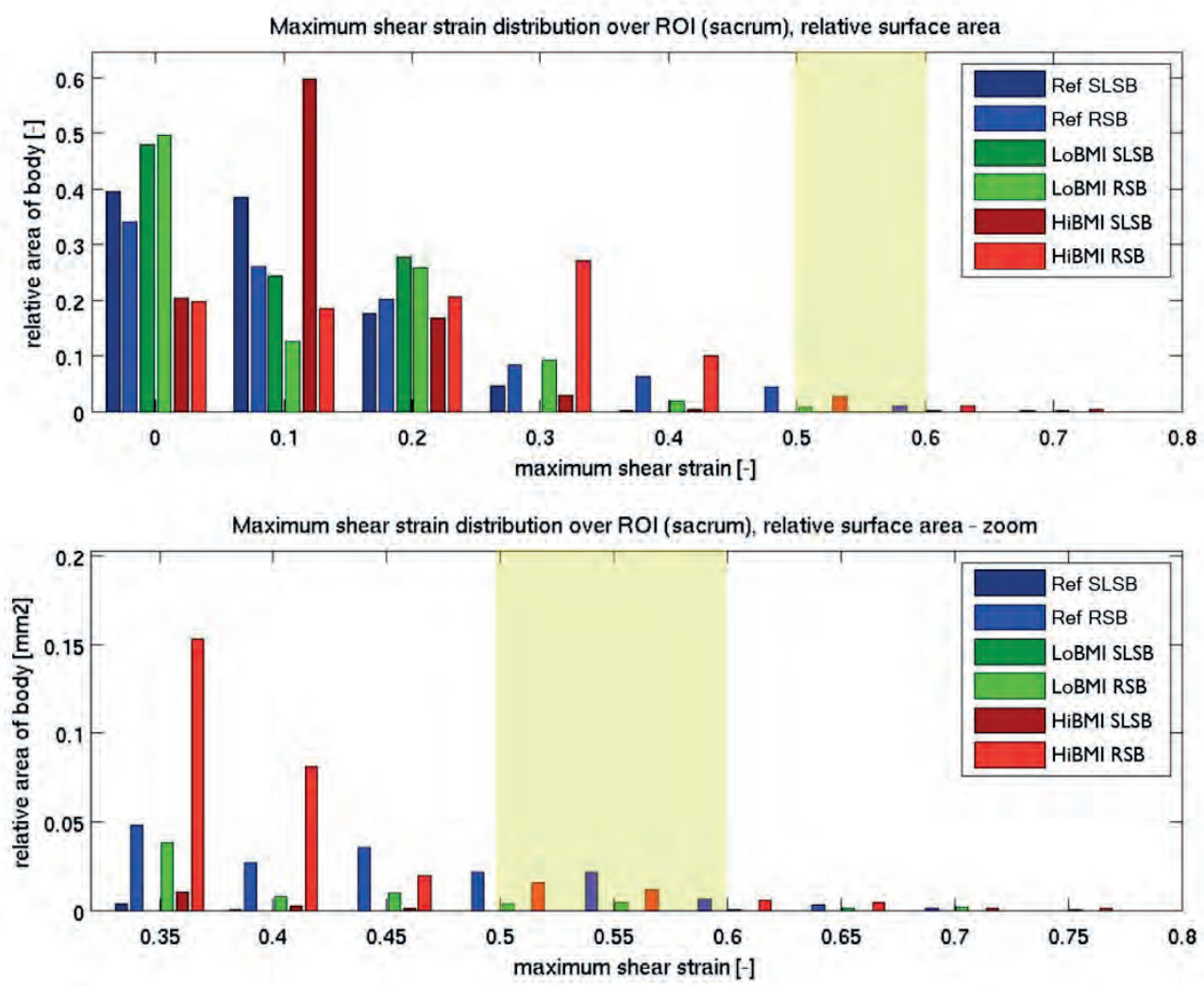

Figure 4.8a-b. Maximum shear strain distribution in all tissues at the sacral region of interest of all three meshes on the soft-layered long spine board (SLSB) and on the rigid spine board (RSB).

\section{Discussion}

Aetiological research has established that in skeletal muscle under strain, there is another damaging process in addition to cell death as a result of ischaemia, a process which has been termed "direct deformation damage". This process is characterised by a very strong correlation between the local levels of strain applied to tissues and the amount of damage observed in both animal studies and in vitro studies. Although the exact mechanisms are not fully clear, it is known that this damage does not occur below a maximum shear strain threshold of between 0.5 and 0.6 (50 to 60\%), whereas exceeding this threshold leads to the first signs of cell damage becoming evident after 
as little as 10 minutes [30]. The time it takes from these early signs to the development of a full wound is not known, but it seems prudent to stay below this threshold strain. Recent research has shown that ischaemic-induced damage can occur [36], although its effect is registered only after several hours [30]. In all three of our subjects, on the rigid spineboard strains of up to 0.8 were found in muscle as well as fat around the sacral bone, suggesting that the use of the rigid spineboard can result in direct deformation damage.

It is clear from our FE estimations that the maximum strains on the soft-layered spineboard did not exceed the deformation damage threshold for any of the three subjects. This implies that transportation of patients on a soft-layered spineboard is fairly safe, at least in terms of preventing deformation damage. However, it should be noted that the threshold for strains to avoid damage will inevitably be lower for patients presenting with for example trauma, shock or unconsciousness. Although ischaemia is a slow process, the periods during which patients are kept on the spineboard are in some cases prolonged, so it is essential to consider each individual patient's sensitivity. Furthermore, the present analysis only focussed on the sacral area, and other studies in our group, involving blood gas tension levels and pressure measurements, have indicated that the shoulder area is particularly vulnerable on the spineboard (unpublished data).

Some remarks can be made on this study. We only examined three participants, all healthy people. Although the stresses exerted on the tissues due to tissue deformation are likely to be independent of underlying diseases, the effect of this deformation on the tissues may very well be influenced by a patient's pre-existent conditions (e.g. diabetes). In this study we only investigated the effect of different surface properties on the degree of deformation. The level of damage caused by this deformation was beyond the scope of our study. In addition, the model has a number of limitations. For example, it is a two-dimensional plane-stress model and does not take into account tissue deformation in the direction perpendicular to the crosssection we modelled. Although it is in principle possible to create 3-D whole-body models, such a process is laborious and time-consuming, and it is difficult to produce patient-specific models. In a similar manner, the material parameter values used in the model for skin, fat and muscle tissues are only based on average values from a limited sample size. On the other hand, all models were analysed with the same data set, based on tuning the reference model to the rigid spineboard. The good agreement between predicted and measured contours with one single data set provides some confirmation of validity of the methodology employed. 


\section{Conclusions}

Maximum shear strains higher than 0.55 (55\%) were found in the sacral area of three subjects with BMls ranging from 19 to 27 , on the rigid spineboard. These strains may lead to direct internal deformation damage to muscle within minutes. On the softlayered spineboard, for all three subjects maximum strains did not exceed the deformation damage threshold. For none of the three subjects did the maximum strains on the soft-layered spineboard exceed the deformation damage threshold. 


\section{References}

1. American College of Surgeons Committee on Trauma. Advanced Trauma Life Support for Doctors. 7th ed. Chicago, IL: American College of Surgeons; 2004.

2. National Association of Emergency Medical Technicians. Prehospital Trauma Life Support, 6th ed. St. Louis, MO: Mosby/JEMS Publications; 2006.

3. Brownlee AE. Managing spinal immobilisation devices in the emergency department. Australas Emerg Nurs J. 2005;8:79-83.

4. Vickery D. The use of the spinal board after the pre-hospital phase of trauma management. Emerg Med J. 2001;18:51-4.

5. Cooke MW. Use of the spinal board within the accident and emergency department. Emerg Med J. 1998;15:108-13.

6. Stagg MJ, \& Lovell ME. A repeat audit of spinal board usage in the emergency department. Injury. 2008;39:323-6.

7. Lerner EB, \& Moscati R. Duration of patient immobilization in the ED. Am J Emerg Med. 2000;18:2830.

8. Sheerin F, \& de Frein R. The occipital and sacral pressures experienced by healthy volunteers under spinal immobilization: a trial of three surfaces. J Emerg Nurs. 2007;33:447-50.

9. Malik MH, \& Lovell ME. Current spinal board usage in emergency departments across the UK. Injury. 2003;34:327-9.

10. Yeung $\mathrm{JHH}, \mathrm{CHeung} \mathrm{NK}$, Graham CA \& Rainer TH. Reduced time on the spinal board-effects of guidelines and education for emergency department staff. Injury. 2006;37:53-6.

11. Cordell WH HJ, Olinger ML, Stroman SJ \& Nelson DR. Pain and tissue-interface pressures during spineboard immobilization. Ann Emerg Med. 1995;26:31-6.

12. Hauswald M, Hsu M \& Stockoff C. Maximizing comfort and minimizing ischemia: a comparison of four methods of spinal immobilization. Prehosp Emerg Care. 2000;4:250-2.

13. Zlupko GR, Megargel RE \& O'Connor RE. Patient discomfort due to indiscriminant use of spinal immobilization. Prehosp Emerg Care. 2004;8:101.

14. Baldwin KM, \& Ziegler SM. Pressure ulcer risk following critical traumatic injury. Adv Wound Care. 1998;11:168-75.

15. Watts D, Abrahams E, MacMillan C, et al. Insult after injury: pressure ulcers in trauma patients. Orthop Nurs. 1998;17:84-91.

16. European Pressure Ulcer Advisory Panel, National Pressure Ulcer Advisory Panel. Prevention and treatment of pressure ulcers: clinical practice guideline. Washington DC; 2009.

17. Allman RM, Goode PS, Burst N, Bartolucci AA \& Thomas DR. Pressure ulcers, hospital complications, and disease severity: impact on hospital costs and length of stay. Adv Wound Care. 1999;12:22-30.

18. Graves N, Birrell FA \& Whitby M. Modeling the economic losses from pressure ulcers among hospitalized patients in Australia. Wound Repair Regen. 2005;13:462-7.

19. Dealey C, Posnett J \& Walker A. The cost of pressure ulcers in the United Kingdom. J Wound Care. 2012;21:261-6.

20. Nijs N, Toppets A, DeFloor T, Bernarts K, Milisenxc K \& van den Berghe G. Incidence and risk factors for pressure ulcers in the intensive care unit. J Clinical Nursing. 2008;18:1258-66.

21. Whittington KT, \& Briones R. National prevalence and incidence study: 6-year sequential acute care data. Adv Skin Wound Care. 2004;17:490-4.

22. Halfens R, Schols J, Meesterberends E, et al. Landelijke Prevalentiemeting Zorgproblemen Rapportage resultaten. (In Dutch) www.lpz-um.eu . 2012.

23. Bouten CVC, Knight MM, Lee DA \& Bader DL. Compressive deformation and damage of muscle cell sub-populations in a model system. Ann Biomed Eng. 2001;29:153-63. 
24. Wang Y-N, Bouten CVC, Lee DA \& Bader DL. Compression induced damage in a muscle cell model in vitro. Eng Med. 2005;219:1-12.

25. Breuls RGM, Bouten CVC, Oomens CWJ, Bader DL \& Baaijens FPT. Compression induced cell damage in engineered muscle tissue: an in vitro model to study pressure ulcer aetiology. Ann Biomed Eng. 2003;31:1357-65.

26. Gawlitta D, Oomens CWJ, Bader DL, Baaijens FPT \& Bouten CVC. Temporal differences in the influence of ischemic factors and deformation on the metabolism of engineered skeletal muscle. J Appl Physiol. 2007;103:464-73.

27. Gefen AG, van Nierop BJ, Bader DL \& Oomens CWJ. Strain-time cell death threshold for skeletal muscle in a tissue-engineered model system for deep tissue injury. J Biomech. 2008;41:2003-12.

28. Bosboom EMH, Bouten CVC, Oomens CWJ, van Straaten H, Baaijens FPT \& Kuipers H. Quantification and localisation of damage in rat muscles after controlled loading; a new approach to study the aetiology of pressure sores. Med Eng Phys. 2001;23:195-200.

29. Ceelen KK, Stekelenburg A, Loerakker S, et al. Compression-induced damage and internal tissue strains are related. J Biomech. 2008;41:3399-404.

30. Loerakker S, Manders E, Strijkers GJ, et al. The effects of deformation, ischemia, and reperfusion on the development of muscle damage during prolonged loading. J Appl Physiol. 2011;111:1168-77.

31. Stekelenburg A, Gawlitta D, Bader DL \& Oomens CWJ. Deep tissue injury: how deep is our understanding? Arch Phys Med Rehabil. 2008;89:1410-3.

32. Linder-Ganz E, Shabshinb N, Itzchak Y, Gefen A. Assessment of mechanical conditions in sub-dermal tissues during sitting: a combined experimental-MRI and finite element approach. J Biomech. 2007;40:1443-54.

33. Hemmes B, Poeze M \& Brink PRG. Reduced tissue-interface pressure and increased comfort on a newly developed soft-layered long spineboard. J Trauma. 2010;68:593-8.

34. Ogden RWGS, \& Sgura I. Fitting hyperelastic models to experimental data. Comput Mech. 2004;34:484-502.

35. Loerakker S, Stekelenburg A, Strijkers GJ, et al. Temporal effects of mechanical loading on deformation-induced damage in skeletal muscle tissue. Ann Biomed Eng. 2010;38:2577-87.

36. Berg G, Nyberg S, Harrison P, Baumchen J, Gurss E \& Hennes E. Near-infrared spectroscopy measurement of sacral tissue oxygen saturation in healthy volunteers immobilizes on rigid spineboard. Prehosp Emerg Care. 2010;14:419-24. 



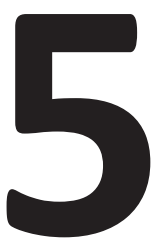

\section{Cytokine IL-1 $\alpha$ as marker for tissue damage in spineboard immobilisation}

A prospective, randomised open-label crossover trial
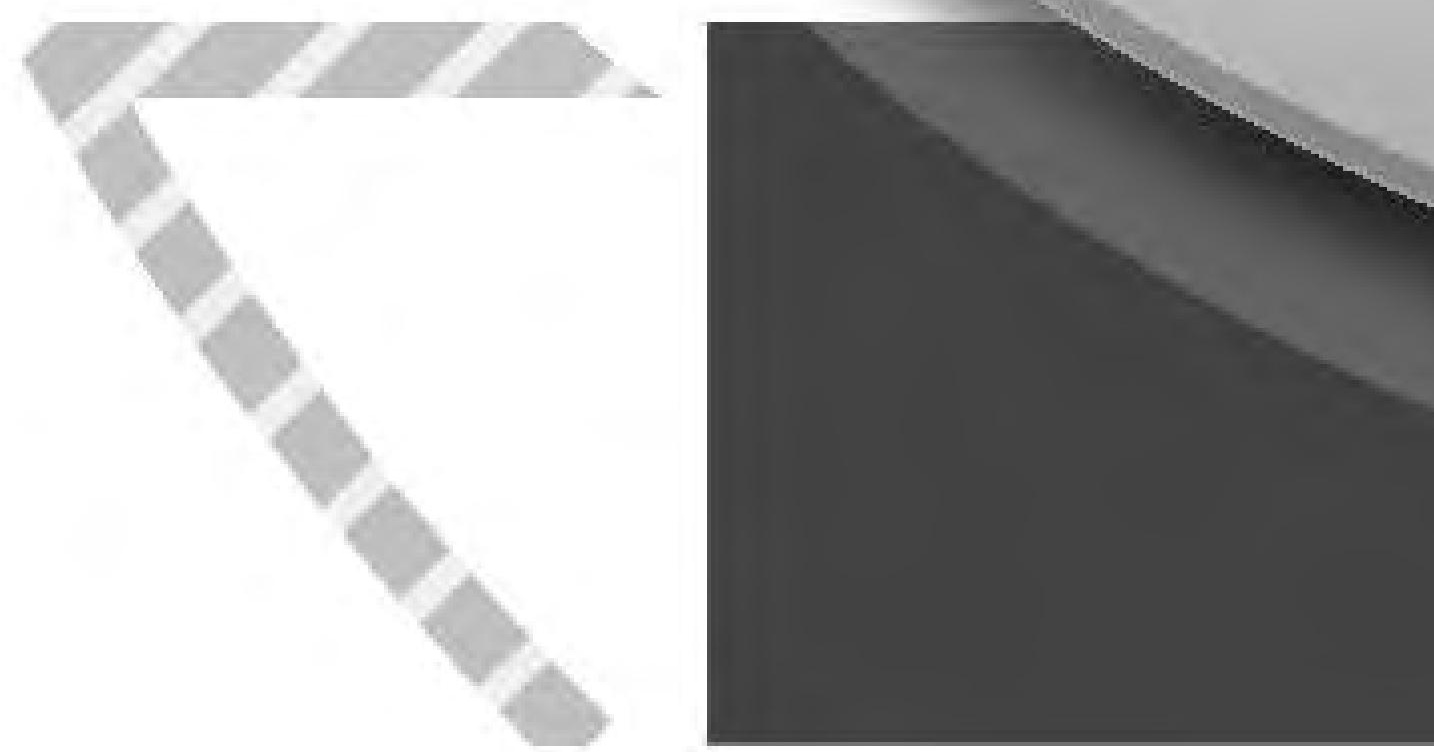

Baukje Hemmes, Luuk A. de Wert, Peter R.G. Brink, Cees W.J. Oomens, Dan L. Bader, Martijn Poeze 


\section{Abstract}

Background: Spinal immobilisation using a rigid long spineboard is a well-established procedure in trauma care. During immobilisation, the body is exposed to high tissueinterface pressures. This may lead to a localised inflammatory response of the skin, which can be used to monitor the body's response to different types of immobilisation device.

Aim: In this study we compared the standard rigid spineboard with a new soft-layered spineboard regarding tissue-interface pressures, reactive hyperaemia and cutaneous IL1 $\alpha$ release.

Methods: Twelve healthy male participants were asked to lie supine on both a rigid and a soft-layered spineboard, loading the sacrum for one hour, followed by one hour in unloaded position. Tissue-interface pressures on the buttocks during loading were measured continuously using a pressure mapping mat. Cutaneous IL1 $\alpha$ concentration was assessed using Sebutapes, during 20-minute periods. After each 20-minute period, a photo of the buttocks was taken, which was later assessed for redness by two observers.

Results: Significant differences in tissue-interface pressure and reactive hyperaemia were found between the two types of spineboard. Release of IL1 $\alpha$ was found to increase with prolonged exposure to pressure, and to decrease in the unloaded prone position. A significant relationship was found between tissue-interface pressure and reactive hyperaemia, but not with IL1 $\alpha$ release. Time course of IL1 $\alpha$ release was similar for both types of spineboard.

Conclusions: IL1 $\alpha$ has a strong relationship with pressure exposure time, but not with pressure magnitude. Furthermore, IL1 $\alpha$ was measured even in the absence of visible redness of the skin. Pressure ulcer prevention through pressure relieve should therefore be a priority for care providers, even in the absence of visible tissue damage. 


\section{Introduction}

The use of spinal immobilisation with long spineboards and cervical collars in the extrication and transfer of trauma patients is a well-established standard precaution in both pre-hospital and in-hospital protocols [1, 2]. These methods are considered particularly essential for unconscious trauma patients, who are unable to maintain spinal alignment by muscle tone, to protect the patients from (further) spinal injury. Although there is a general consensus that the patient should be removed from the spineboard as soon as reasonably possible $[1,3,4]$, the average time patients are reported to spend on a spineboard has been estimated to be approximately one hour [5-7] and may be markedly longer in some cases [6, 8]. Prolonged immobilisation on the spineboard causes significant discomfort and pain [9], and on occasion may cause pressure ulcers to develop adjacent to bony prominences [8]. These ulcers are painful [10-12] and debilitating for the patient $[13,14]$ and take a long time to heal [15-17], resulting in prolonged hospitalisation [18, 19] and reduced quality of life [20-23].

Pressure ulcers result from oxygen and/or nutrient deprivation and cell damage caused by pressure [24-26], either starting superficially at the skin or starting from deeper soft tissue layers adjacent to bone prominences and then extending to the skin. Lying on a rigid surface compromises the ability of the soft tissues (skin, muscles, fat) to adapt to the shape of the surface, resulting in high tissue-interface pressures [27], and may result in high compressive and shear strains in the tissues [28]. High tissueinterface pressures may lead to closure of capillaries, thereby depriving tissues of oxygen and other vital nutrients, which will eventually lead to cell damage Furthermore, the high strains themselves may cause cell damage directly [29, 30], adding to the risk of developing pressure ulcers. This cell damage triggers an inflammatory response, which involves the release of cytokines into the skin, such as IL1 $\alpha$, IL-1RA and IL-8. Earlier studies [31, 32] showed that not only can IL1 $\alpha$ be detected after relatively short periods of loading time ( $<2$ hours), its release is also related to the magnitude of pressure, and a relation with pressure ulcers has been found in in vivo studies [33, 34]. We therefore decided to focus on IL1 $\alpha$ as an objective indicator of tissue damage in this study. Cytokines can be collected non-invasively in humans with the use of Sebutapes $[32,35,36]$, self-adhesive patches specifically developed to absorb sebum, which can yield a number of proteins including cytokines. Previous research by our group showed that lying on a rigid spineboard results in high tissueinterface pressures [27], increased reactive hyperaemia [37] and increased tissue strains at the sacrum [28]. The hypothesis for the present study was that lying on a rigid spineboard would result in an elevated release of IL1 $\alpha$ as a result of the increased tissue-interface pressures when compared to lying on a soft-layered spineboard. In 
addition, we hypothesised that there would be a relationship between the pressureinduced reactive hyperaemia and the IL $1 \alpha$ concentration.

We compared the IL1 $\alpha$ response in healthy volunteers during and after exposure to the increased tissue-interface pressures on a standard rigid spineboard and a softlayered spineboard, in a prospective randomised open-label crossover study.

\section{Methods}

\section{Study design}

We performed a prospective, randomised open-label crossover study evaluating tissue-interface pressures, redness of the skin, and IL1 $\alpha$ production, comparing a standard rigid long spineboard and a soft-layered long spineboard, using healthy volunteers. The study was approved by the Medical Research Ethics Committee of the Maastricht University Medical Centre and registered as NTR4537 in the Dutch Primary Registry.

\section{Participants}

Sample size for this study was estimated to be a minimum of 12 persons [38]. We therefore recruited 12 volunteers through advertisements and word of mouth recruitment. Inclusion criteria were male sex, age 20-30 years, BMI $19-25 \mathrm{~kg} / \mathrm{m} 2$ and Caucasian ethnicity. Exclusion criteria were a history of pressure ulcers and skin conditions such as eczema, rashes or psoriasis with local expression on the buttocks/sacrum. Written informed consent was obtained from all participants before enrolment in the study.

\section{Materials and interventions}

Participants were randomly assigned to the order in which the two devices were to be tested. Testing took place in two separate sessions. Two different support devices designed for prehospital trauma care were used: a standard long spineboard (FernoWashington, Wilmington, $\mathrm{OH}$ ), and a prototype soft-layered long spineboard [27, 28, 37]. All tests were done in the same climate-controlled room, which was kept at a temperature of $23.2 \pm 0.4{ }^{\circ} \mathrm{C}$. Each session started with a 15 -minute acclimatisation period, in which the participant was not allowed to put load on the buttocks (i.e. no sitting or lying on the back).

The area of interest surrounding the bony prominence of the sacrum was marked out using a surgical pen. At the end of the acclimatisation period, four Sebutapes were placed on the skin in the marked-out area (Figure 5.1), and fixed in place using a custom-made pressure-roller [39]. After one minute, each Sebutape was retrieved, 
placed in a coded vial and stored on dry-ice. The Sebutapes were then replaced and the participant lay supine on the spineboard for another 20 minutes, after which the Sebutapes were once again replaced. This procedure was repeated three times, allowing for a total loaded period of 1 hour. The procedure was then repeated three more times at 20-minute intervals while the buttocks remained unloaded, to achieve reperfusion. Participants were allowed to either stand or lie prone during this second hour. At the end of the session, the vials with Sebutapes were stored in a $-80^{\circ} \mathrm{C}$ freezer until further processing. The second session was planned a week later, and at this session the other device was tested in a manner identical to that used in the first session.

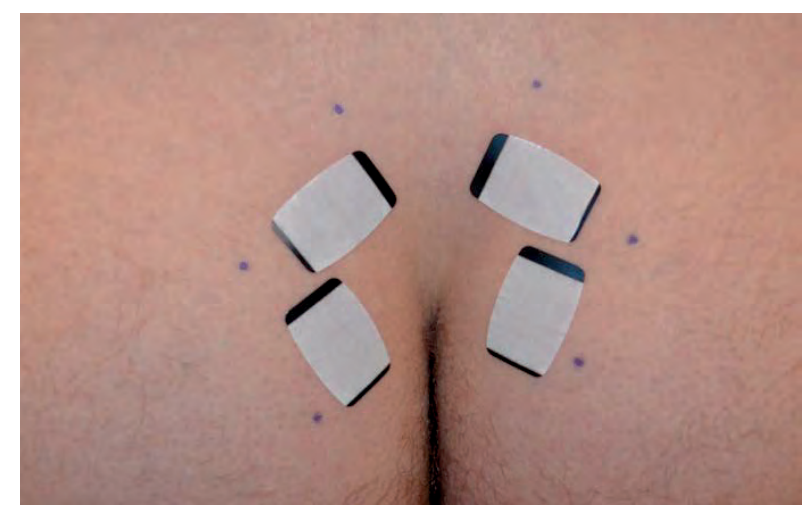

Figure 5.1. Four Sebutapes in place in the marked-out area of interest on the sacrum/buttocks for baseline measurement.

The Sebutape extraction process was based on a study by Perkins et al [36]. Briefly, the vials with tapes were thawed to room temperature, after which $2 \mathrm{~mL}$ phosphate buffered saline was added to each vial. After 1 hour, the tapes were sonicated for 10 $\min$ at $20^{\circ} \mathrm{C}$, vigorously vortexed for $2 \mathrm{~min}$ and additionally mixed with a pipette tip. IL$1 \alpha$ concentration in the samples was measured using a commercially available human IL-1 $\alpha$ / IL1F1 enzyme-linked immunosorbent assay (ELISA) kit (DuoSet R\&D system) with a detection range of $3.9-250 \mathrm{pg} / \mathrm{mL}$. IL-1 $\alpha$ levels below the detection threshold were recorded as zero for statistical testing. The amount of total protein (TP) collected on each Sebutape was measured using a Thermo Scientific TM Micro BCA Protein Assay Kit (Pierce Biotechnology) with a detection range of $0.5-20 \mu \mathrm{g} / \mathrm{mL}$. To correct for sebum uptake by the Sebutapes, IL-1 $\alpha /(\mathrm{TP})$ - ratio was calculated as IL-1 $\alpha$ concentration $(\mathrm{pg} / \mathrm{mL})$ divided by the total protein $(T P)$ concentration $(\mu \mathrm{g} / \mathrm{mL})$. Tissueinterface pressures were continuously measured using a Tekscan Conformat model 5330 pressure-mapping device (Tekscan Inc, Boston,MA, USA). This system consists of 
a thin, easily foldable seat-sized pressure-mapping mat, equipped with 1024 capacitive sensors. This mat was placed between the subject and the spineboard at the level of the buttocks. Pressure on the sensors generates a voltage difference, which increases linearly with the pressure. The mat was connected to a laptop computer with special Tekscan software (version 7.20), for real-time pressure monitoring.

After each 20-minute period, the back of each participant was visually inspected and the skin redness was semi-quantitatively graded. Any redness was tested for being blanchable using a clear plastic disc, so as to distinguish between blanchable and nonblanchable erythema [40]. Photographs of the buttocks were made to permit an independent second reviewer to quantify and qualify the presence of redness.

\section{Outcome measures}

IL1 $\alpha$ in $\mathrm{pg} / \mathrm{mL}$ was measured at baseline and subsequently after every 20-minute period. Total protein (TP) was measured in $\mu \mathrm{g} / \mathrm{mL}$ at the same time points. IL1 $\alpha$ /TP ratio was used as a standardised measure to compensate for inter-individual excretion differences.

Pressures in $\mathrm{mmHg}$ were recorded continuously during the loaded period for each sensor in the pressure mapping mat. Peak Pressure Index (PPI) in $\mathrm{mmHg}$ was defined as the area of $3 \times 3$ sensors which included the sensor showing the peak pressure and which had the highest average pressure including this sensor. The area was identified based on the first recording of every 20 -minute period in the loaded condition. PPI can be considered more reliable than peak pressure for assessing the pressure-distributing capabilities of a support surface, because it examines a $19.5-\mathrm{cm} 2$ surface area in addition to the peak pressure registered by one single sensor, which has a surface of only $2.16 \mathrm{~cm} 2$ [41]. Pressures were analysed for the areas covered by the Sebutapes, as well as for the sacral location with the highest pressure, regardless of the location.

As a clinical measure of tissue response, redness of the skin was scored after every 20-minute period, and recorded as absent, diffuse or clearly defined, and further categorised in terms of blanchable or non-blanchable. Redness of the skin was assessed independently by two of the authors (BH and PB) blinded for the spineboard allocation.

\section{Statistical analysis}

Statistical analysis was performed using the Statistical Package for Social Sciences (SPSS, IBM), version 20.0.0.1. Pressures and IL1 $\alpha$ values are presented as mean \pm SD; redness scores are presented in categories. Statistical analysis of tissue-interface pressures and II $1 \alpha$ was performed using repeated-measures analysis of variance with correction of the degrees of freedom using the Greenhouse-Geisser estimates of sphericity when Mauchly's test indicated that the assumption of sphericity had been 
violated. The analysis used time as the within-group factor and type of spineboard as the between-group factor. Post-hoc group comparisons at the different time points were only performed when the overall repeated-measures tests were statistically significant. All scores were tested for normality using the Kolmogorov-Smirnov test. Parametric variables were compared using Student's t-test, while non-parametric variables were compared using the Mann-Whitney $U$ statistic and Spearman rank correlation. Nominal variables were compared across independent groups using the chi-squared test or Fisher's exact test. Homogeneity of variance was assessed using Levene's test. Level of significance was set at $P<0.05$.

\section{Results}

\section{Tissue-interface pressures}

Mean tissue-interface pressures and pressure indexes were higher on the rigid spineboard than on the soft-layered spineboard, for all participants, all Sebutape locations and all time points ( $\left.P_{S}<0.05\right)$ except for the tissue-interface pressure in the top right location during first time period $(P<0.1)$ (Figures 5.2 and 5.3; Tables 5.1 and 5.2).

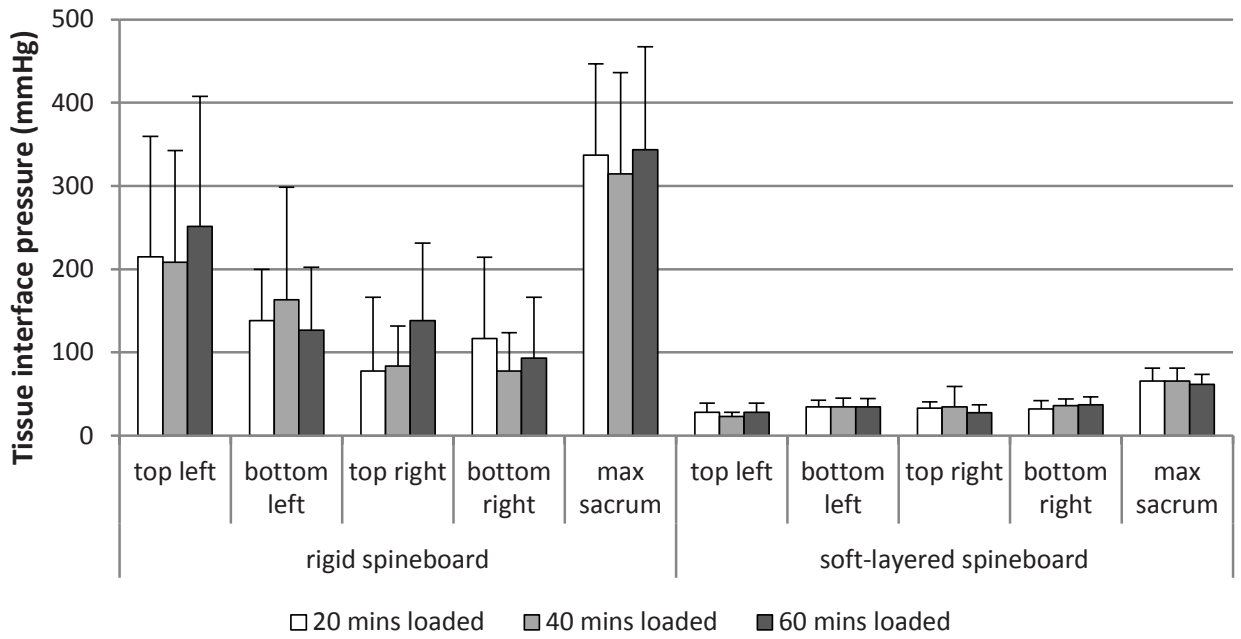

Figure 5.2. Tissue-interface pressure in $\mathrm{mmHg}$ in relation to location and time point. Presented data are means + standard deviation 


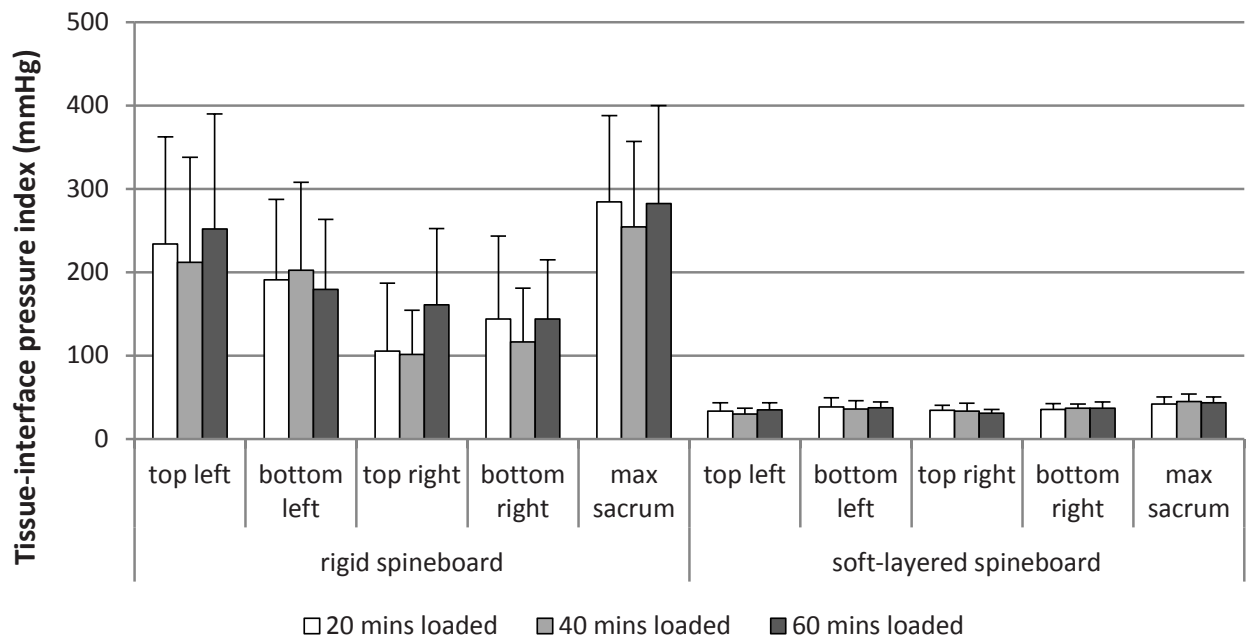

Figure 5.3. Tissue-interface pressure index in $\mathrm{mmHg}$ in relation to location and time point.

Presented data are means + standard deviation

Table 5.1. Mean \pm standard deviation peak pressure and Peak Pressure Index in $\mathrm{mmHg}$ for the rigid spineboard, in relation to location and duration of loading

\begin{tabular}{|c|c|c|c|c|c|c|}
\hline$\overline{\text { Rigid }}$ & Peak press & & & Peak press & e index & \\
\hline Location & $\begin{array}{l}20 \text { mins } \\
\text { loaded }\end{array}$ & $\begin{array}{l}40 \text { mins } \\
\text { loaded }\end{array}$ & $\begin{array}{l}60 \text { mins } \\
\text { loaded }\end{array}$ & $\begin{array}{l}20 \text { mins } \\
\text { loaded }\end{array}$ & $\begin{array}{l}40 \text { mins } \\
\text { loaded }\end{array}$ & $\begin{array}{l}60 \text { mins } \\
\text { loaded }\end{array}$ \\
\hline Top left & $215 \pm 144$ & $208 \pm 134$ & $251 \pm 157$ & $234 \pm 128$ & $212 \pm 126$ & $252 \pm 138$ \\
\hline Bottom left & $138 \pm 62$ & $163 \pm 135$ & $127 \pm 76$ & $191 \pm 96$ & $202 \pm 106$ & $180 \pm 84$ \\
\hline Top right & $78 \pm 89$ & $84 \pm 48$ & $138 \pm 93$ & $105 \pm 82$ & $102 \pm 53$ & $161 \pm 91$ \\
\hline Bottom right & $117 \pm 98$ & $78 \pm 46$ & $93 \pm 73$ & $144 \pm 99$ & $117 \pm 64$ & $144 \pm 71$ \\
\hline Max sacrum & $337 \pm 110$ & $314 \pm 122$ & $344 \pm 124$ & $284 \pm 104$ & $254 \pm 102$ & $282 \pm 117$ \\
\hline
\end{tabular}

Table 5.2. Mean \pm standard deviation peak pressure and Peak Pressure Index in $\mathrm{mmHg}$ for the soft-layered spineboard, in relation to location and duration of loading

\begin{tabular}{|c|c|c|c|c|c|c|}
\hline \multirow{2}{*}{$\begin{array}{l}\text { Soft-layered } \\
\text { spineboard } \\
\text { Location }\end{array}$} & \multicolumn{3}{|c|}{ Peak pressure } & \multicolumn{3}{|c|}{ Peak pressure index } \\
\hline & $\begin{array}{l}20 \text { mins } \\
\text { loaded }\end{array}$ & $\begin{array}{l}40 \text { mins } \\
\text { loaded }\end{array}$ & $\begin{array}{l}60 \text { mins } \\
\text { loaded }\end{array}$ & $\begin{array}{l}20 \text { mins } \\
\text { loaded }\end{array}$ & $\begin{array}{l}40 \text { mins } \\
\text { loaded }\end{array}$ & $\begin{array}{l}60 \text { mins } \\
\text { loaded }\end{array}$ \\
\hline Top left & $28 \pm 11$ & $23 \pm 5$ & $28 \pm 11$ & $34 \pm 10$ & $30 \pm 7$ & $35 \pm 8$ \\
\hline Bottom left & $35 \pm 8$ & $34 \pm 10$ & $34 \pm 10$ & $39 \pm 11$ & $36 \pm 10$ & $38 \pm 7$ \\
\hline Top right & $33 \pm 8$ & $34 \pm 25$ & $27 \pm 10$ & $35 \pm 6$ & $34 \pm 9$ & $31 \pm 5$ \\
\hline Bottom right & $32 \pm 10$ & $36 \pm 8$ & $37 \pm 10$ & $35 \pm 7$ & $37 \pm 5$ & $37 \pm 7$ \\
\hline Max sacrum & $66 \pm 15$ & $66 \pm 15$ & $62 \pm 12$ & $42 \pm 9$ & $45 \pm 9$ & $44 \pm 7$ \\
\hline
\end{tabular}

On the rigid spineboard, mean tissue-interface pressures and pressure indexes differed between the Sebutape locations at all time point $(P s<0.05)$, but remained constant over time, with the exception of pressures and pressure index at the top right location, 
which showed a significant increase in the third time period $(P<0.05)$. On the softlayered spineboard, pressures and pressures indexes were similar across locations and time points $\left(P_{S}>0.05\right)$.

On the rigid spineboard, mean tissue-interface pressures and pressure indexes at the sacral area were higher than those to the right of the sacrum $\left(P_{S}<0.05\right)$. On the soft-layered spineboard, maximum pressures at the sacrum were higher than those measured at the four Sebutape locations $(P s<0.01)$.

\section{IL1 $\alpha$ /TP ratio}

The IL1 $\alpha$ /TP ratio showed a similar pattern over time on both the soft-layered and the rigid spineboard, with ratios increasing during the loading period and decreasing during the unloaded period (Figure 5.4). On both spineboards, the ratio increased significantly between baseline measurement and the first loading period $\left(P_{S}<0.01\right)$. The decrease in the ratio after the first period of unloading was also significant ( $P S<$ 0.05 ) for both spineboards. In an earlier pilot study with the rigid spineboard, we used a tape-stripped section of the forearm as a positive control for IL1 $\alpha$ secretion and nontape-stripped, non-loaded skin of the forearm as a negative control. Both conditions showed a comparable slight increase in IL1 $\alpha$ secretion, similar to the effects seen on the sacrum (data not shown).

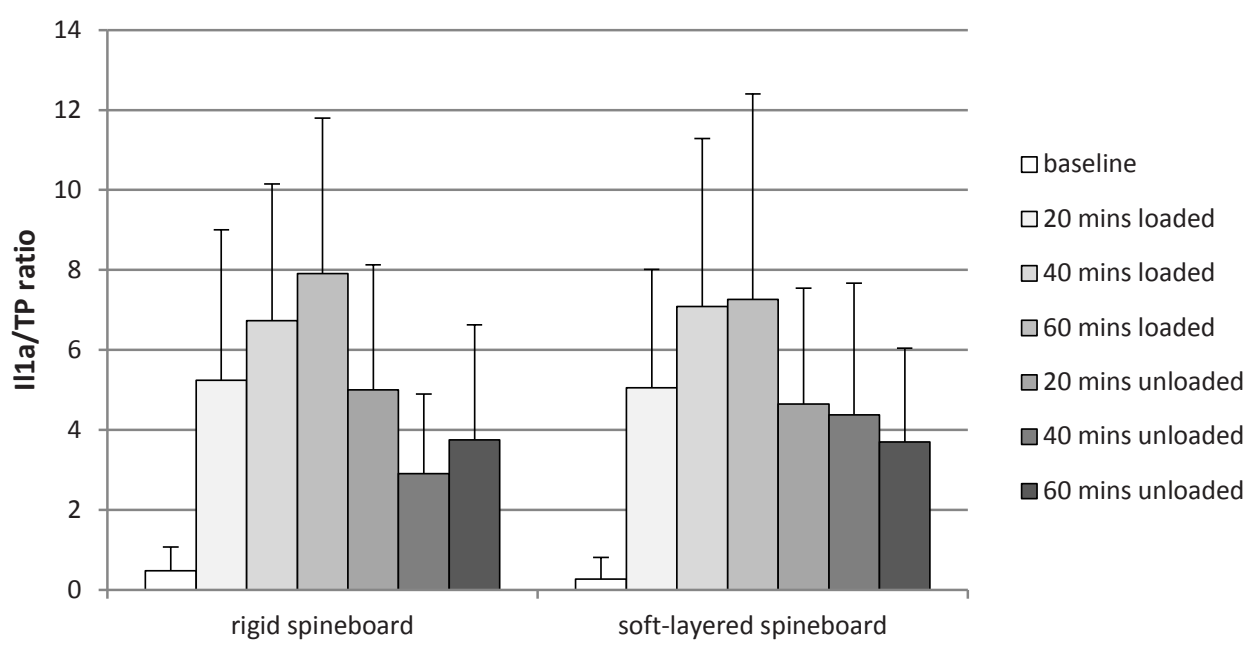

Figure 5.4. IL1 $\alpha$ /TP ratio, average of the four locations, comparing the two devices over time. Presented data are means + standard deviation 


\section{Skin redness}

Log-linear regression analysis revealed a significant difference between the two spineboards in the intensity and course of pressure-induced redness. In particular, the soft-layered spineboard generally caused no or only diffuse redness after several periods of loading, while the rigid spineboard demonstrated clearly defined redness even after the first period of loading $(\chi 2(1)=23.24, P<0.01$; Figure 5.5).

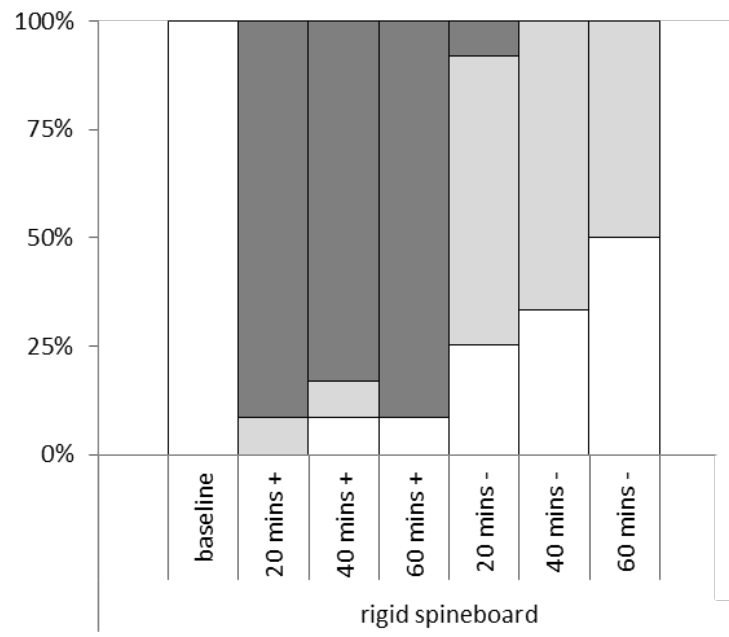

$\square$ no redness

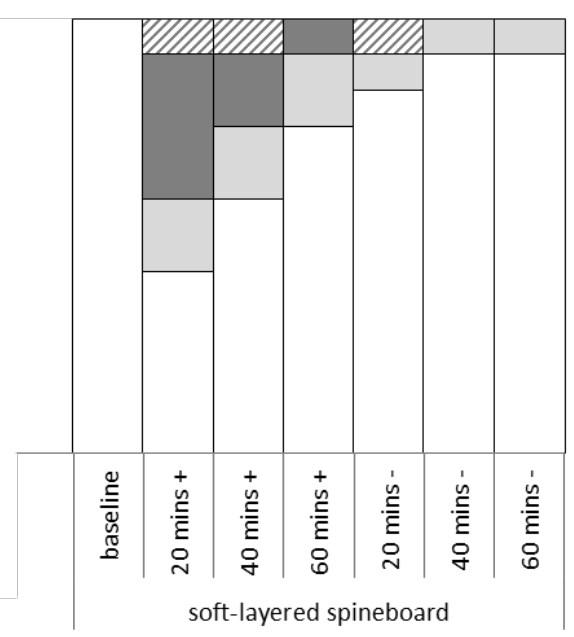

$\square$ clearly defined redness

$\square$ other

Figure 5.5. Redness of the buttocks in relation to time point and device +: loaded: -: unloaded.

Other: red streak within the intergluteal cleft, no redness on the buttocks

Complete agreement of redness level was found in $77 \%$ of the cases, resulting in a kappa of 0.61 . Disagreement was mostly seen in the assessment of no redness versus diffuse redness (13\%). All redness was blanchable.

\section{IL-1 $\alpha$ /TP ratio in relation to pressure}

Neither on the rigid nor on the soft-layered spineboards was there a significant correlation between tissue-interface pressures and IL1 $\alpha /$ TP ratio $(R=0.017, P=1.0$ and $R=0.070 ; P=0.4$ respectively; all locations summarised; Figure 5.6). 


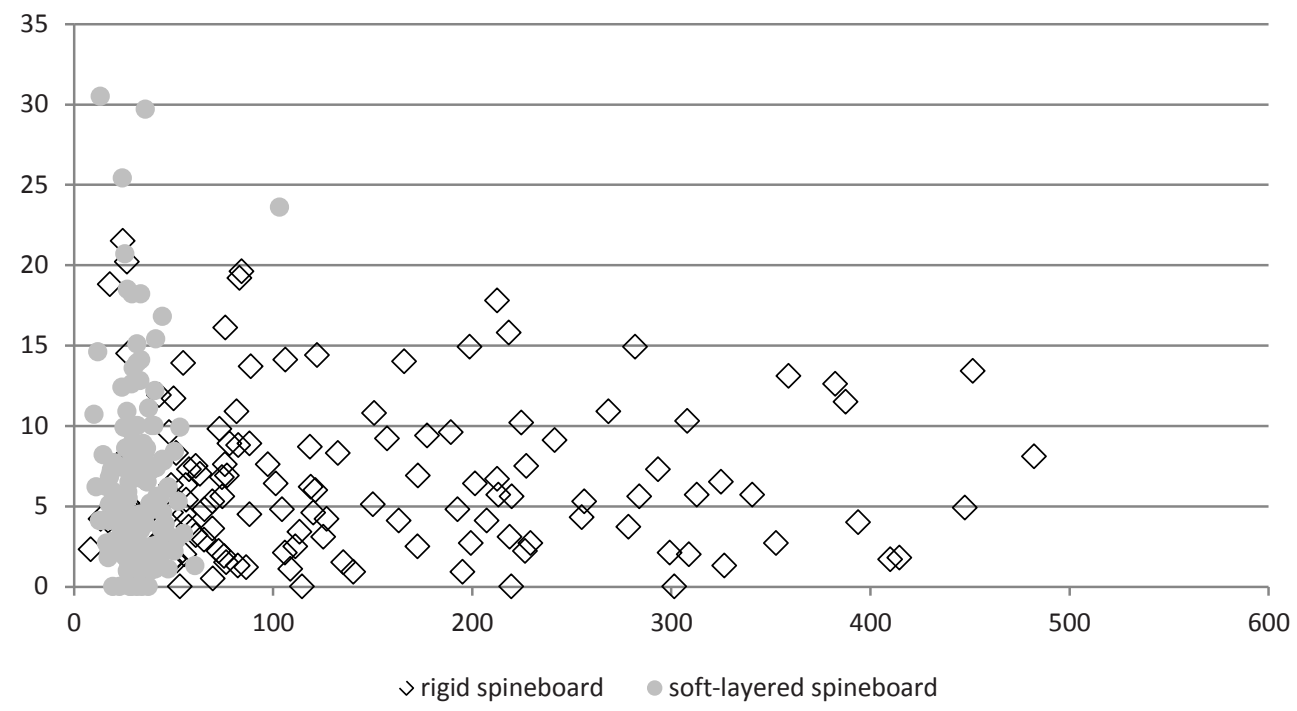

Figure 5.6. Peak pressure versus IL1 $\alpha$ /TP ratio

Peak pressure in $\mathrm{mmHg}$ (x-axis) vs IL1 $\alpha /$ TP ratio (y-axis), all measurements

\section{IL-1 $\alpha$ /TP ratio in relation skin redness}

For both the rigid spineboard and the soft-layered spineboards, a significant correlation was found between redness and IL1 $\alpha$ /TP ratio $(R=0.634, P<0.01$ and $R=$ $0.286, P<0.05$, respectively; Figure 5.7).

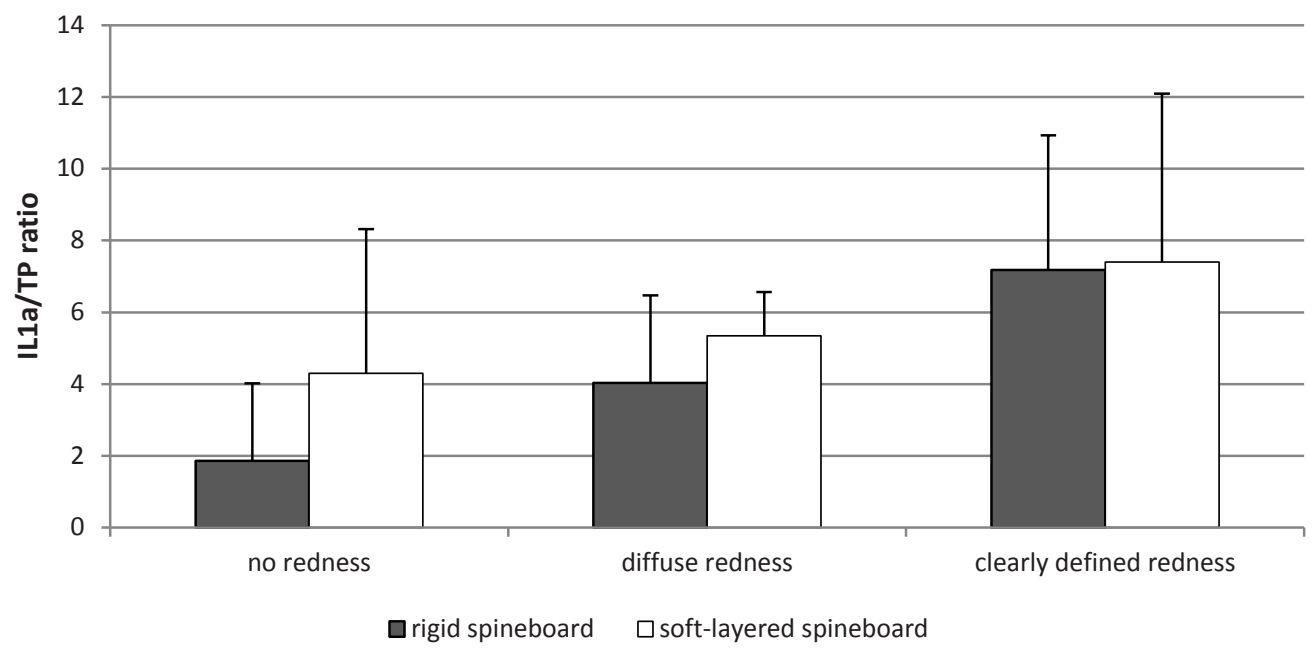

Figure 5.7. IL1 $\alpha$ /TP ratio in relation to the two devices and level of redness Presented data are means + standard deviation 


\section{Pressure in relation to skin redness}

A significant relation was found between tissue-interface pressure and skin redness, with higher pressures correlating with higher levels of skin redness $(R=0.707, P<$ 0.01).

\section{Discussion}

This prospective, randomised open label crossover study examined the relation between tissue-interface pressure, IL1 $\alpha$ secretion and redness of the skin in persons lying on two types of spineboard. We found marked differences between the effects of the rigid spineboard and the soft-layered spineboard with regard to tissue-interface pressure and redness of the skin. However, IL1 $\alpha$ /TP ratios were similar for both types of spineboard and did not correlate with the tissue-interface pressures.

To our knowledge, this is the first study to relate IL1 $\alpha$ /TP ratio to tissue-interface pressures in a clinically relevant scenario. Earlier studies have used various pressures applied to engineered skin constructs [31, 32, 35]. In accordance with our earlier study [37], we found a clear relationship between tissue-interface pressure and redness of the skin on both types of spineboard. The traditional rigid spineboard showed mean tissue-interface pressures up to five times higher than the corresponding values measured on the soft-layered spineboard. Pressures were measured across the entire sacral area, including the four sites where the Sebutapes were placed. These local pressures were recorded in order to identify the local correlation between pressure and IL1 $\alpha$ /TP ratio. In addition, we determined the peak pressure in the sacral area, to test for global effects of pressure. No clear correlations were found between either the local or the global assessment of the tissue-interface pressures and IL1 $\alpha$ /TP ratio. On the other hand, we did find a clear relationship between the level of skin redness and the IL $1 \alpha$ /TP ratio. It appears that either IL1 $\alpha$ release is initiated at a lower pressure threshold than the pressure needed to induce reactive hyperaemia, or IL1 $\alpha$ is upregulated as an epiphenomenon. Unfortunately, research evidence for either mechanism remains sparse.

As shown in an earlier study from our group [45], the rigid spineboard induces high levels of stress and strain forces on the soft tissues, whereas these effects are significantly less on the soft-layered spineboard. The real danger of the rigid spineboard may therefore be in damage to the deeper tissue layers. Although IL1 $\alpha$ may not be the right biomarker to indicate deep tissue injury, other markers such as lactate and mFABp are released in response to ischemic muscle damage [46-48], and could be used as indicators of this specific type of tissue damage. 
A few remarks should be made about the methods used in this study. First, we only looked at IL1 $\alpha$ as a biochemical marker for tissue damage. Although this cytokine is known to be related to pressure $[32,35]$, our results show no specific relationship with the magnitude of pressure over a specific loading period. Other biomarkers may therefore be more suitable to serve as objective markers of tissue damage in relation to the magnitude of tissue-interface pressures. Furthermore, measurements in the loaded condition were performed in three periods of 20 minutes, with a short unloaded period of two minutes in between to replace the Sebutapes and photograph the buttock area. During these short breaks, skin redness was a sign of reperfusion. It is possible that this brief reperfusion period affected cytokine excretion. Finally, redness of the buttocks was assessed from photographs displayed on a computer screen, and the settings of the screen may have made it difficult to discriminate between no redness and diffuse redness, as assessed by the two judges.

\section{Conclusion}

IL1 $\alpha$ has a strong relationship with pressure exposure time, but not with pressure magnitude. Furthermore, IL1 $\alpha$ was measured even in the absence of visible redness of the skin. Pressure ulcer prevention through pressure relieve should therefore be a priority for care providers, even in the absence of visible tissue damage. 


\section{References}

1. American College of Surgeons Committee on Trauma. Advanced Trauma Life Support for Doctors. $9^{\text {th }}$ ed. Chicago, IL: American College of Surgeons; 2012.

2. National Association of Emergency Medical Technicians, Prehospital Trauma Life Support. $7^{\text {th }}$ ed; 2010,Burlington, MA: Jones \& Bartlett Learning.

3. Vickery $D$, The use of the spinal board after the pre-hospital phase of trauma management. Emerg Med J. 2001. 18:51-54.

4. Ahn H, Singh J, Nathens A et al. Pre-hospital care management of a potential spinal cord injured patient: a systematic review of the literature and evidence-based guidelines. J Neurotraum. 2011. 28:1341-1361.

5. Cooney DR, Wallus H, Asaly M \& Wojcik S. Backboard time for patients receiving spinal immobilization by emergency medical services. Int J emerg Med. 2013. 6:17-19.

6. Lerner EB, \& Moscati R. Duration of patient immobilization in the ED. Am J Emerg Med. 2000. 18: 2830.

7. Yeung JHH, Cheung NK, Graham CA \& Rainer TH. Reduced time on the spinal board - effects of guidelines and education for emergency department staff. Injury. 2006. 37:53-56.

8. Schouten R, Albert $T$ \& Kwon BK. The spine-injured patient: initial assessment and emergency treatment. J Am Acad Orthop Surg. 2012. 20: 336-346.

9. Kwan I \& Bunn F. Effects of prehospital spinal immobilization: a systematic review of randomized trials on healthy subjects. Prehosp Disaster Medicine. 2005. 20: 47-53.

10. Gunes UY. A descriptive study of pressure ulcer pain. Ostomy Wound Manag. 2008. 54: 56-61.

11. Pieper B, Langemo D \& Cuddigan J. Pressure ulcer pain: a systematic literature review and National Pressure Ulcer Advisory Panel white paper. Ostomy Wound Manag. 2009. 55: 16-31.

12. Rastinehad D. Pressure ulcer pain. J Wound Ostomy Cont. 2006. 33: 252-257.

13. Hopkins A, Dealey C, Bale S, DeFloor T \& Worboys F. Patient stories of living with a pressure ulcer. J Adv Nurs. 2006: 345-353.

14. Fox C. Living with a pressure ulcer: a descriptive study of patients' experiences. Br J Community Nurs. 2002. 7: 11-22.

15. Bennett G, Dealey C \& Posnett J. The cost of pressure ulcers in the UK. Age Ageing. 2004. 33: $230-235$.

16. Chapman BR, Mills KJ, Pearce LM, \& Crowe TC. Use of an arginine-enriched oral nutrition supplement in the healing of pressure ulcers in patients with spinal cord injuries: an observational study. Nutr \& Diet. 2011. 68: 208-213.

17. Sanada H, lizaka S, Matsui $Y$, et al. Clinical wound assessment using DESIGN-R total score can predict pressure ulcer healing: pooled analysis from two multicenter cohort studies. Wound Repair Regen. 2011. 19: 559-567.

18. Allman RM, Damiano AM \& Strauss MJ. Pressure ulcers, hospital complications, and disease severity: impact on hospital costs and length of stay. Adv Skin Wound Care. 1999. 12: 22-30.

19. Graves N, Birrell FA \& Whitby M. Modeling the economic losses from pressure ulcers among hospitalized patients in Australia. Wound Repair Regen. 2005. 13:465-467.

20. Essex HN, Clark M, Sims J, Warriner A \& Cullum N. Health-related quality of life in hospital inpatients with pressure ulceration: assessment using generic health-related quality of life measures. Wound Repair Regen. 2009. 17: 797-805.

21. Gorecki C, Brown JM, Nelson EA, et al. Impact of pressure ulcers on quality of life in older patients: a systematic review. J Am Geriatr Soc. 2009. 57: 1175-1183.

22. Langemo DK, Melland H, Hanson D, Olson B \& Hunter S. The lived experience of having a pressure ulcer: a qualitative analysis. Adv Skin Wound Care. 2000. 13: 225-235. 
23. Spilsbury K, Nelson A, Cullum N, Iglesias C, Nixon J \& Mason S. Pressure ulcers and their treatment and effects on quality of life: hospital inpatient perspectives. J Adv Nurs. 2007. 57: 494-504.

24. DeFloor T. The risk of pressure sores: a conceptual scheme. J Clin Nurs. 1999. 8:206-216.

25. Luscombe MD \& Williams JL. Comparison of a long spinal board and vacuum mattress for spinal immobilisation. Emerg Med J. 2003. 20: 476-478.

26. Mak AFT, Yu Y, Kwan LPC, Sun L \& Tam EWC. Deformation and reperfusion damages and their accumulation in subcutaneous tissues during loading and unloading: a theoretical modeling of deep tissue injuries. J Theor Biol. 2011. 11:65-73.

27. Hemmes B, Poeze M \& Brink PRG. Reduced tissue-interface pressure and increased comfort on a newly developed soft-layered long spineboard. J Trauma. 2010. 68:593-598.

28. Oomens CWJ, Zenhorst W, Broek M, et al, A numerical study to analyse the risk for pressure ulcer development on a spine board. Clin Biomech. 2013. 28:736-742.

29. Loerakker S, Manders E, Strijkers GJ, et al. The effects of deformation, ischemia, and reperfusion on the development of muscle damage during prolonged loading. J Appl Physiol. 2011. 111: 1168-1177.

30. Oomens CWJ, Loerakker S, \& Bader DL. The importance of internal strains as opposed to interface pressures in the prevention of pressure related deep tissue injury. J Tissue Viabil. 2010. 19:35-42.

31. Bronneberg D, Bouten CVC, Oomens CWJ, van Kemenade PM \& Baaijens FPT. An in vitro model system to study the damaging effects of epidermal loading. Ann Biomech Eng. 2006. 34: 506-514.

32. Cornelissen LH, Bronneberg D, Bader DL, Baaijens FPT \& Oomens CWJ. The transport profile of cytokines in epidermal equivalents subjected to mechanical oading. Ann Biomech Eng. 2009. 37: 10071018.

33. Bronneberg D. Biochemical markers for early detection of superficial pressure ulcers; chapter 6: In vivodetection of grade 1 pressure ulcers; PhD thesis, 2007, Eindhoven University of Technology: Eindhoven, the Netherlands.

34. Lans van der M. Biochemical marker for early and objective detection of grade 1 pressure ulcers; MSc thesis, 2007, Eindhoven University of Techonology: Eindhoven, the Netherlands.

35. Bronneberg D, Spiekstra SW, Cornelissen LH, et al. Cytokine and chemokine release upon prolonged mechanical loading of the epidermis. Exper Dermatol. 2007. 16:567-573.

36. Perkins MA, Osterhues MA, Farage MA \& Robinson MK. A noninvasive method to assess skin irritation and compromised skin conditions using simple tape adsorption of molecular markers of inflammation. Skin Res Technol. 2001. 7: 227-237.

37. Hemmes B, Brink PRG \& Poeze M. Effects of unconsciousness during spinal immobilization on tissueinterface pressures: A randomized controlled trial comparing a standard rigid spineboard with a newly developed soft-layered long spineboard. Injury. 2014. 45:1741-1746.

38. Julious SA. Sample size of 12 per group rule of thumb for a pilot study. Pharm Stat. 2005. 4: 287-298.

39. Bronneberg D. Biochemical markers for early detection of superficial pressure ulcers; Ch. 6, 2007, Eindhoven University of Technology, Dept. of Biomedical Technology: Eindhoven, the Netherlands.

40. Vanderwee K, Grypdonck MHF, de Bacquer D \& Defloor T. The reliability of two observation methods of nonblanchable erythema, grade1 pressure ulcer. Appl Nurs Res. 2006. 19:156-162.

41. Keller BPJA, van Overbeeke J \& van der Werken Chr. Interface pressure measurement during surgery: a comparison of four operating Table surfaces. J Wound Care. 2005. 15:5-9.

42. European Pressure Ulcer Advisory Panel; National Pressure Ulcer Advisory Panel, Prevention and treatment of pressure ulcers: clinical practice guideline2009, Washington DC (http://www.npuap.org).

43. Gawlitta D, Oomens CWJ, Bader DL, Baaijens FPT \& Bouten CVC. Temporal differences in the influence of ischemic factors and deformation on the metabolism of engineered skeletal muscle. J Appl Physiol. 2007. 103:464-473.

44. Ceelen KK, Stekelenburg A, Loerakker S et al. Compression-induced damage and internal tissue strains are related. J Biomech. 2008. 41:3399-3404. 


\section{Chapter 5}

45 Oomens CWJ, Zenhorst W, Broek M, et al. A numerical study to analyse the risk for pressure ulcer development on a spine board. Clin Biomech. 2013. 28:736-742

46. Glatz JFC, Kleine AH, van Nieuwenhoven FA et al. Fatty-acid-binding protein as a plasma marker for the estimation of myocardial infarct size in humans. Br Heart J. 1994. 71: 135-140.

47. Pelsers MMAL, Hernems WT \& GLatz JFC. Fatty acid-binding proteins as plasma markers of tissue injury. Clin Chim Acta. 2005. 352:15-35.

48. Knight SL, Taylor RP, Polliack A \& Bader DL. Establishing predictive indicators for the status of loaded soft tissues. J Appl Physiol. 2001. 90: 2231-2237. 


\section{Effect of spineboard and headblocks on the image quality of head CT scans}

Baukje Hemmes, Cécile R.L.P.N. Jeukens, Alia Al-Haidari, Paul A.M. Hofman, Ed S. van der Linden, Peter R.G. Brink, Martijn Poeze 


\section{Abstract}

Background: Trauma patients at risk for spinal injury are transported to the hospital using immobilisation devices such as a vacuum mattress or spineboard with or without headblocks. Trauma resuscitation protocols dictate that these devices have to remain in place until the spine is cleared from injury, which often means that CT scans are made with the devices in place. These devices may however decrease radiological image quality and even hamper diagnosis. The aim of this study was to determine the influence of various spinal stabilisation devices on visual image quality.

Methods: Image quality was judged for both patient CT scans and phantom CT scans. The phantom set-up comprised (1) a vacuum mattress without headblocks; (2) a rigid spineboard without headblocks, with standard soft-foam headblocks, or with newdesign headblocks; or (3) a soft-layered spineboard without headblocks, with standard soft-foam headblocks, or with new-design headblocks. CT scans of 217 patients were assessed retrospectively by two radiologists who gave a visual score for image quality, scoring both the quantity and impact of artefacts caused by the immobilisation devices. CT scans using a head phantom were assessed for the number and extent of artefacts.

Results: Overall, artefacts were found in $67 \%$ of CT scans of patients on immobilisation devices, and these hampered diagnosis in $10 \%$ of cases. In the phantom CT scans, artefacts were present in all set-ups with one or more devices present, and were seen in $20 \%$ of all scan slices. The presence of headblocks resulted in more artefacts in both the patient and phantom CT scans.

Conclusions: Immobilisation devices result in decreased radiological image quality in CT scans of the head. Considerable efforts should therefore be made to adjust the design of the immobilisation devices, and to enable removal of the headblocks before CT scans are made. 


\section{Introduction}

For trauma patients at risk of spinal injury or brain injury, immobilisation using a spineboard and headblocks or a vacuum mattress has become the gold standard in prehospital care, including the transport of patients to hospital [1-4]. Although physicians are advised to remove these devices as soon as possible [1-3], many patients undergo clinical and radiological evaluation in the emergency department and/or radiology department to rapidly assess life-threatening injuries while still on the device $[5,6]$, and removal of the device is often postponed until after x-ray and CT imaging has been done $[4,5]$.

In recent years, devices for spinal immobilisation have received renewed attention, focusing on patient comfort or discomfort $[7,8]$ and functional restrictions [9-11], resulting in some devices being redesigned. Together with the traditional devices such as the rigid spineboard and the vacuum mattress, there is now a wide range of devices for spinal immobilisation available to choose from. However, all of these devices are made of plastics and foam, and contain cut-outs or, in the case of the vacuum mattress, folds, which can create artefacts on CT scans [12-14], hampering identification or exclusion of pathology. A number of studies have described how misinterpreting artefacts as pathology can affect patient treatment [15-18].

To our knowledge no study has yet been published comparing a vacuum mattress and spineboards plus headblocks in terms of their effect on image quality. We therefore examined the number of artefacts caused by the various devices, using both patient and phantom scans. We also assessed how often these artefacts hamper diagnosis in clinical practice. We hypothesised that the soft foam headblocks and the rigid spineboard would cause more objective and subjective image quality loss than the more recently designed headblocks and spineboards and the vacuum mattress.

\section{Materials and methods}

\section{Patient data}

We retrospectively studied the data of 241 consecutive patients who sustained a blunt trauma in 2011, and underwent standardised automatic exposure-controlled (AEC) multidetector CT of the brain during their initial resuscitation. Approval was obtained from the Medical Research Ethics Committee of the Maastricht University Medical Centre with informed consent waived, and it was registered as ISRCTN 68626238. Depending on the nature of the accident and other (potential) injuries, the patients were presented for CT scan lying on a rigid spineboard (Millennia Backboard; FernoWashington, Wilmington, $\mathrm{OH}$ ), a prototype soft-layered spineboard [7], a vacuum 
mattress (RedVac, Radstadt, Austria) or no device, with either 26-cm standard softfoam headblocks (universal head immobilizer; Ferno-Washington Inc, Wilmington, $\mathrm{OH}), 19 \mathrm{~cm}$ new-design headblocks (speedblocks; Laerdal Medical, Stavanger, Norway) or no headblocks. Patients with incomplete data $(n=24)$ were excluded from the analysis, resulting in a total of 217 patients with complete data.

All patient CT scans were made using a Philips Brilliance 64 (Philips, Best, the Netherlands) with tube potential $120 \mathrm{kV}$, effective current time $179 \mathrm{mAs}$, beam collimation $64 \times 0.9 \mathrm{~mm}$ and AEC enabled. The presence of artefacts was judged using the $5 \mathrm{~mm}$ reconstructions of the scans. Artefacts were defined as any disturbances of visual image quality, such as lines or streak artefacts, due to objects located between the radiation beam and the receiver. Artefacts which could be attributed to (medical) implants or devices other than those for spinal immobilisation were not scored in this study.

In the $5 \mathrm{~mm}$ reconstructions that were judged to show artefacts, the original 1 $\mathrm{mm}$ scans were used to assess the impact of the artefacts on clinical judgement. If the presence or exclusion of pathology could not be conclusively ascertained on the $1 \mathrm{~mm}$ scans due to the presence of artefacts, these artefacts were scored as hampering judgement. All scans were judged by a radiologist, and a random sample of 20 scans was assessed by a second radiologist blinded for the first observer's assessment.

\section{Phantom data}

CT scans were made of the various devices for spinal immobilisation in combination with a head phantom (density equal to water). All phantom scans were made using a Philips Brilliance 64 (Philips, Best, the Netherlands) with tube potential 120kV, effective tube current $179 \mathrm{mAs}$, beam collimation $64 \times 0.9 \mathrm{~mm}$ and AEC disabled. All slices were assessed as to whether artefacts were present or not. An experienced trauma surgeon and an experienced radiologist both judged all scans.

\section{Statistical analyses}

Statistical analysis was performed using the Statistical Package for Social Sciences (SPSS; IBM Chicago, IL), version 20.0.0. Non-parametric data were compared using Kruskal-Wallis testing for overall differences, with significance assumed at $\mathrm{P}<0.05$, and Mann-Whitney $U$ test for differences between devices, with significance after Bonferroni correction assumed at $(\mathrm{P}<0.05 /$ number of tests). Parametric data were compared using one-way ANOVA for differences between devices, with significance assumed at $\mathrm{P}<0.05$. 


\section{Results}

\section{Patient data}

Of the 217 patients analysed, 68\% were male, and median age was 48 years (range, 0 95; standard deviation 21.9). Most patients (65\%) were scanned lying on a rigid spineboard with new-design headblocks. Eleven per cent of patients were scanned lying on the soft-layered spineboard with or without headblocks, and $12 \%$ of patients were scanned without any device in place (Table 6.1). A review of electronic patient files showed that $31 \%$ of patients had abnormalities of the face, skull and/or brain diagnosed on CT.

The percentage of trauma patients scans with artefacts varied between $40 \%$ and $78 \%$, depending on the devices used (Table 6.1). Figures $6.1 \mathrm{a}$ and $6.1 \mathrm{~b}$ show two examples of typical artefacts encountered. Artefacts were found in $40-50 \%$ of the scans with only a spineboard or vacuum mattress and no headblocks, and in $70-78 \%$ of the scans with a spineboard plus headblocks. Interrater reliability was high (Cronbach's alpha $=0.81, r=0.68$ ) for the number of scans with an artefact. One-way independent ANOVA showed significant differences between the groups $(F 7,209=3.678, P<0.01)$. Overall, the artefacts hampered clinical judgement in $10 \%$ of the scans. In four patients, no conclusive diagnosis could be made due to the artefacts, although this did not change the treatment of the head trauma.

Table 6.1. Artefacts in CT scans of the head of trauma patients $(n=217)$

\begin{tabular}{llll}
\hline Set-up & $\begin{array}{l}\text { Number of } \\
\text { patients }\end{array}$ & $\begin{array}{l}\text { Scans with } \\
\text { artefacts }\end{array}$ & $\begin{array}{l}\text { Scans with artefacts } \\
\text { hampering clinical } \\
\text { judgement }\end{array}$ \\
\hline No spineboard, no headblocks & 25 & $9(36 \%)$ & $1(4 \%)$ \\
No spineboard, standard soft-foam headblocks & 5 & $2(40 \%)$ & $0(-)$ \\
No spineboard, new-design headblocks & 0 & - & - \\
Rigid spineboard, no headblocks & 12 & $5(42 \%)$ & $1(8 \%)$ \\
Rigid spineboard, standard soft-foam headblocks & 10 & $7(70 \%)$ & $1(10 \%)$ \\
Rigid spineboard, new-design headblocks & 140 & $106(76 \%)$ & $15(11 \%)$ \\
Soft-layered spineboard, no headblocks & 5 & $2(40 \%)$ & $0(-)$ \\
Soft-layered spineboard, standard soft-foam headblocks & 18 & $14(78 \%)$ & $2(11 \%)$ \\
Soft-layered spineboard, new-design headblocks & 0 & - & - \\
Vacuum mattress & 2 & $1(50 \%)$ & $1(50 \%)$ \\
Overall & $217(100 \%)$ & $146(67 \%)$ & $21(10 \%)$ \\
\hline
\end{tabular}



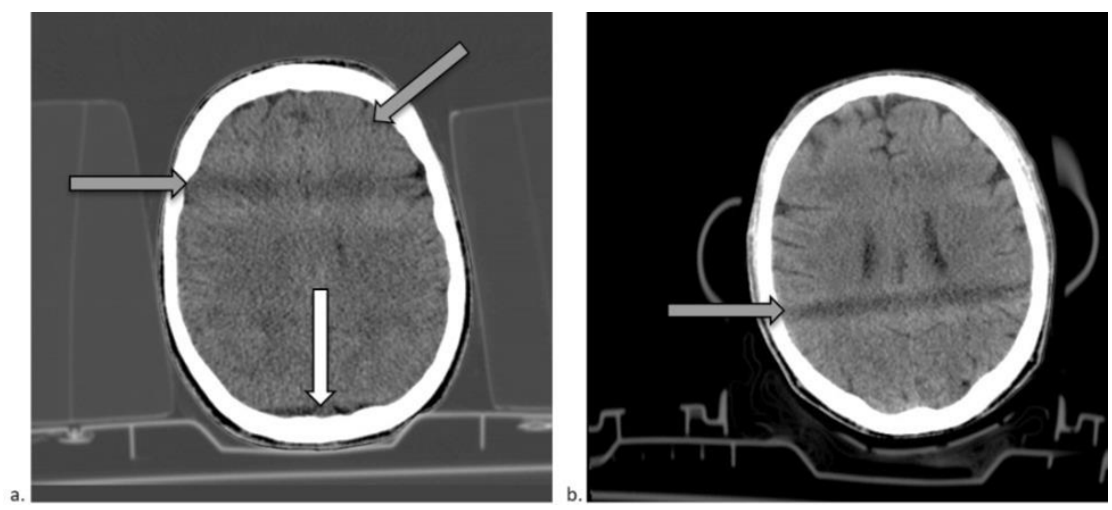

Figure 6.1a-b. Patient CT scans with artefacts caused by headblocks. a. Rigid spineboard plus soft foam headblocks. b. Rigid spineboard plus new-design headblocks.

Grey arrows: shadow caused by material-to-air transition in the headblocks; white arrow: line artefact cause by the base plate of the headblocks.

\section{Phantom data}

In the phantom study, a mean of one in every five CT scan slices was affected by artefacts, with percentages ranging from $0 \%$ to $68 \%$ of slices depending on the immobilisation devices used (Table 6.2). Figures $6.2 \mathrm{a}$ and $6.2 \mathrm{~b}$ show examples of typical artefacts. Kruskal-Wallis testing for non-parametric data showed a significant overall effect for differences between set-ups in terms of artefact percentages $(H(6)=$ 484.9, $P<0.001)$. Mann-Whitney tests with Bonferroni correction indicated that using headblocks significantly increased the number of slices with artefacts for both spineboards, with the standard soft-foam headblocks producing more artefacts than the new-design headblocks. The vacuum mattress caused significantly fewer artefacts than either of the spineboards plus soft-foam headblocks. Interrater reliability was high for the presence of artefacts (Cronbach's alpha $=0.88$ with $r=0.78$ ). The majority of differences of judgement were related to the extent of the artefacts.

Table 6.2. Artefacts in the phantom study; judgement of slices with artefacts

\begin{tabular}{lllll}
\hline Condition & $\begin{array}{l}\text { Number } \\
\text { of slices }\end{array}$ & $\begin{array}{l}\text { Slices with } \\
\text { artefacts }\end{array}$ & alpha & $\mathbf{R}$ \\
\hline Rigid spineboard, no headblocks & 168 & $0(0 \%)$ & $*$ & $* *$ \\
Rigid spineboard, standard soft-foam headblocks & 151 & $103(68 \%)$ & 0.75 & $0.61(p<0.01)$ \\
Rigid spineboard, new-design headblocks & 196 & $28(14 \%)$ & 0.88 & $0.79(p<0.01)$ \\
Soft-layered spineboard, no headblocks & 170 & $1(1 \%)$ & $*$ & $* *$ \\
Soft-layered spineboard, standard soft-foam headblocks & 172 & $105(61 \%)$ & 0.80 & $0.66(p<0.01)$ \\
Soft-layered spineboard, new-design headblocks & 165 & $18(11 \%)$ & 0.98 & $0.97(p<0.01)$ \\
Vacuum mattress, no headblocks & 178 & $8(4 \%)$ & $*$ & $* *$ \\
Overall & 1200 & $262(22 \%)$ & 0.88 & $0.78(p<0.01)$ \\
\hline
\end{tabular}

* alpha cannot be calculated because the scale had zero variance for one or both of the observers

** correlation cannot be calculated because at least one of the variables is constant 

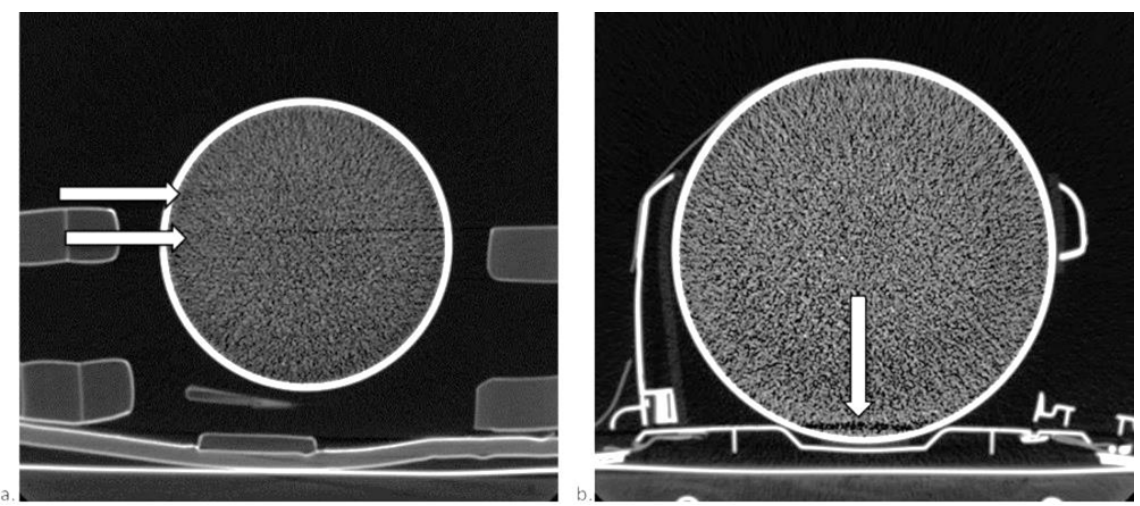

Figure 6.2a-b. Phantom CT scans with artefacts caused by headblocks. a. rigid spineboard plus soft-foam headblocks; arrows indicate artefacts caused by material-to-air transition of the headblocks; b. rigid spineboard plus new-design headblocks; arrow indicates artefact caused by base plate.

\section{Discussion}

Our results show that devices used for spinal immobilisation negatively influence image quality in CT scans of the head. The presence of headblocks, especially the softfoam headblocks, resulted in the greatest degradation of image quality in both the patient and phantom scans. In the patient scans, the presence of artefacts hampered clinical judgement in $10 \%$ of cases with artefacts, although in none of the cases did this result in a treatment change.

Earlier studies have investigated the influence of immobilisation devices on image quality. Miller et al [13] showed that x-rays of the thorax in trauma patients can be difficult to interpret due to artefacts caused by the spineboard. Schou et al [14] compared seven different types of vacuum mattress using an x-ray phantom, and concluded that the artefacts caused by these devices may obscure fracture lines. Loewenhardt et al [19] compared nine weight-bearing devices for spinal immobilisation using a CTDI phantom, judging the devices according to severity of the artefacts. They concluded that neither a rigid spineboard identical to the one used in our study nor the vacuum mattress produced artefacts. Although we found similar results in our phantom study, the CT scans of actual patients did show artefacts attributable to the rigid spineboard. These findings indicate that in addition to evaluating image quality using a phantom model, assessment of clinical importance of artefacts in patient CT scans is necessary to evaluate the impact on visual image quality.

Artefacts caused by headblocks are an underrated issue and have been mentioned only sporadically [20]. Our study was the first to systematically evaluate the 
impact of headblocks on the radiological image quality. We found the headblocks to add significantly to the number of artefacts seen on CT scans.

Both Du Plessis [17] and Mathur [18] presented cases showing how artefacts in CT scans of the brain can result in unnecessary additional diagnostics and medical treatment. In our series, four patients had their diagnosis adjusted based on results of the follow-up scan, although treatment remained unchanged.

In our study, all devices caused artefacts in the patient CT scans. Since these artefacts have been shown to hamper clinical judgement in some cases, efforts should be made to avoid them. This can be achieved by removing the devices before the CT scan is made, or by improving the radiological properties of the devices. Implementation of changes in emergency care protocols remains difficult [2, 21, 22], indicating that advocating removal of the devices cannot be used as the sole means of reducing the occurrence of artefacts in CT scans. Another option to limit artefacts is to improve the devices by maximising radio translucency, minimising sharp corners and reducing material-to-air transitions at the location of the head.

Some comments can be made on this study. Two important issues in medical radiation are image quality and radiation dose. Although this study only looked at image quality, it is important to recognise the influence the devices have on the radiation dose patients are exposed to. The devices absorb part of the radiation, thereby causing the image dose to be automatically increased, although there is also a certain shielding effect. These influences of the devices have been discussed in detail elsewhere [23]. In the present study, we focused on image quality in relation to immobilisation devices. A direct comparison of the various immobilisation devices for the same patient would be desirable but is not feasible in trauma care. We therefore studied a sufficient number of patients to allow interindividual comparison, and made a direct comparison between the devices using a phantom. The studies were nonblinded. Although the observers were not informed explicitly which immobilisation devices were present, this could be deduced from the CT images. However, artefacts were also found in the CT scans made without any devices present, and agreement between the observers was high. Finally, all CT scans were performed using the same Philips Brilliance 64 scanner. Although it could be argued that the type of scanner affects visual image quality, this impact will have been limited in the present study. The type of artefact described in our study is caused by beam hardening due to energy absorption by the devices. This explains why using a spineboard combined with headblocks results in more artefacts than using a spineboard without headblocks. The difference in the extent of artefacts between the soft foam and new-design headblocks depends on the size of the devices, with the larger $26-\mathrm{cm}$ soft foam headblocks causing artefacts over more slices than the 19-cm new-design headblocks. 
In view of these innate factors affecting the occurrence of artefacts, the use of other CT scanners will likely yield similar outcomes.

\section{Conclusion}

Removing the headblocks before making a CT scan of the head significantly improves both objective and subjective image quality. Considerable efforts should therefore be made to adjust the design of the immobilisation devices, and to enable removal of the headblocks before CT scans are made. 


\section{References}

1. Cooke MW. Use of the spinal board within the accident and emergency department. J Accid Emerg Med. 1998. 15:108-113.

2. Lerner EB \& Moscati R. Duration of patient immobilization in the ED. Am J Emerg Med. 2000. 18:2830.

3. Malik MHA \& Lovell ME. Current spinal board usage in emergency departments across the UK. Injury. 2003. 34:327-329.

4. Stagg MJ \& Lovell ME. A repeat audit of spinal board usage in the emergency department. Injury. 2008. 39:323-326.

5. El-Khoury GY, Kathol MH \& Daniel WW. Imaging of acute injuries of the cervical spine: value of plain radiography, CT, and MR Imaging. Am J Roentgenol. 1995. 164: 43-50.

6. France JC, Bono CM \& Vaccaro AR. Initial radiographic evaluation of the spine after trauma: when, what, where, and how to image the acutely traumatized spine. J Orthop Trauma. 2005. 19:640-649.

7. Hemmes B, Poeze M, \& Brink PRG. Reduced tissue-interface pressure and increased comfort on a newly developed soft-layered long spineboard. J Trauma. 2010. 68:593-598.

8. Oomens CWJ, Zenhorst W, Broek $\mathrm{M}$, et al. A numerical study to analyse the risk for pressure ulcer development on a spine board. Clin Biomech. 2013. 28:736-742.

9. Ay D, Aktas C, Yesilyurt S, Sarikaya S, Cetin A, \& Ozdogan ES. Effects of spinal immobilization devices on pulmonary function in healthy volunteer individuals. Ulus Travma Acil Cerrahi Derg (Turkish Journal of Trauma and Emergency Surgery). 2011. 17:103-107.

10. Ng I, Lim J \& Wong HB. Effects of head posture on cerebral hemodynamics; its influences on intracranial pressure, cerebral perfusion pressure and cerebral oxygenation. Neurosurg. 2004. 54: 593-598.

11. White CC, D.R., Millin MG and the Standards and Clinical Practice Committee. National Association of EMS Physicians, EMS spinal precautions and the use of the long backboard - resource document to the position statement of the national association of EMS physicians and the American College of Surgeons committee on trauma. Prehosp Emerg Care. 2014. 18:306-314.

12. Daffner RH \& Khoury MB. Pseudofractures due to Nec-Loc cervical immobilization collar. Skeletal Radiol. 1987. 16:460-462.

13. Miller JA, Mele C \& Abu-Judeh H. Significance of backboard artifacts on portable trauma series chest radiographs. Emerg Radiol. 1999. 6:334-338.

14. Schou J, Kiermayer H, Ummenhofer W \& Herion H-P. In search of the most suitable technique for truncal spinal immobilization with associated radiography. Eur J Emerg Med. 2001. 8:89-92.

15. Hayes M, Andronikou S, Prabhu S, \& Schulze O. Multiplanar angled reconstructions of reconstructions - a dangerous practice using multidetector CT. Pediatr Radiol. 2006. 36:881-883.

16. Wells IT \& Manghat N. Re: A CT reconstruction artefact that mimics acute subdural haemorrhage. Clin Radiol. 2009. 64:1136-1137.

17. du Plessis A-M, Theron S \& Andronikou S. The effects of misinterpretation of an artefact on multidetector row CT scans in children. Pediatr Radiol. 2009. 39:137-141.

18. Mathur S, Gadde S \& Sunder Koteyar S. Artefact misinterpretation on CT images of the head. Clin Radiol. 2008. 63:1277-1279.

19. Loewenhardt B, Huttinger R, Reinert $M$ et al. Dose effects and image quality: Is there any influence by bearing devices in whole-body computed tomography in trauma patients? Injury. 2014. 45:170-175.

20. Johnson RJ \& Bury RW. Cervical spine radiology. Anaesthesia \& Intensive Care Medicine, 2011. 12: 504-506.

21. Hauswald M. A re-conceptualisation of acute spinal care. Emerg Med J. 2013. 30:720-723. 
22. Hauswald M \& Braude D. Diffusion of medical progress: early spinal immobilization in the Emergency Department. Acad Emerg Med. 2007. 14:1087-1089.

23. Hemmes B, Jeukens CRLPN, Kemerink GJ, Brink PRG \& Poeze M. Effect of spinal immobilization devices on radiation exposure in conventional radiography and computed tomography. Submitted. 



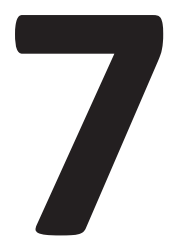

Effect of spinal immobilisation devices on radiation exposure in conventional radiography and computed tomography

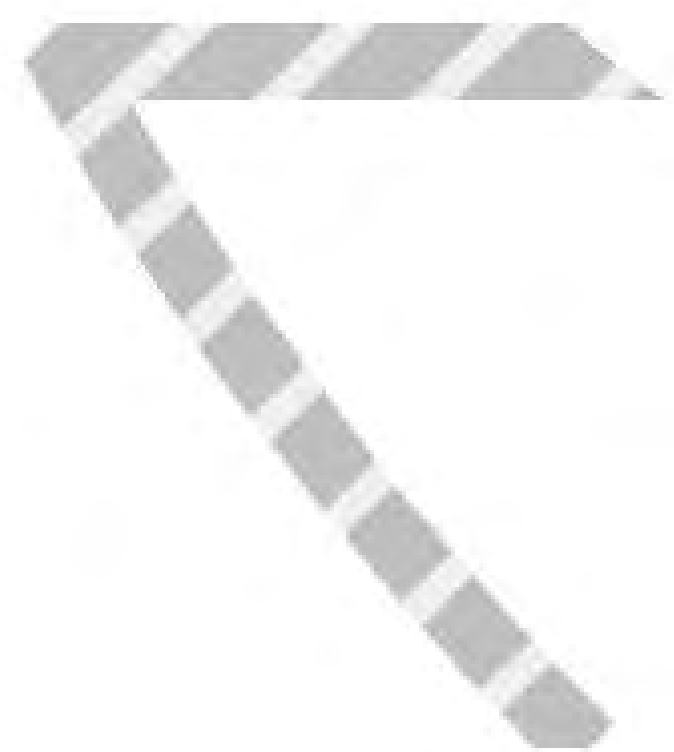

Baukje Hemmes, Cécile R.L.P.N. Jeukens, Gerrit J. Kemerink, Peter R.G. Brink, Martijn Poeze 


\section{Abstract}

Background: Trauma patients at risk for, or suspected of, spinal injury are frequently transported to hospital using full spinal immobilisation. At the emergency department, immobilisation is often maintained until radiological work-up is completed. In this study we examined how these devices influence radiation exposure and noise, as a proxy for objective image quality.

Methods: Conventional radiographs (CR) and computer tomography (CT) scans were made using a phantom immobilised on two types of spineboard and a vacuum mattress, and using two types of headblocks. Images were compared for radiation transmission and quantitative image noise.

Results: In CR up to $23 \%$, and in CT up to $11 \%$ of radiation was blocked by the devices. Without compensation for the decreased transmission, noise increased by up to $16 \%$ in $\mathrm{CT}$, depending on the device used. Removing the headblocks led to a statistically significant improvement in transmission with automatic exposure control (AEC) enabled.

Conclusions: Physicians should make an informed decision whether the increased radiation exposure outweighs the risk of missing a clinically significant injury by not making a CR or CT scan. Manufacturers of immobilisation devices should take radiological properties of their devices into account in the development and production process. 


\section{Introduction}

The spineboard was originally introduced as a means of extricating patients from a crashed vehicle or from a hard-to-reach location [1, 2]. Over the decades, spinal immobilisation using a spineboard or vacuum mattress with a head immobiliser has become the gold standard for prehospital care for trauma patients, including transport of patients on the spineboard into the hospital [3-6]. Although physicians are advised to remove patients from these devices as soon as possible [3-5], many patients undergo primary clinical and radiological evaluation at the emergency department and/or radiology department to rapidly assess life-threatening injuries with the devices in place $[7,8]$. Furthermore, whole-body computed tomography (CT) has recently come to be used as a primary diagnostic investigation at the emergency department. Secondary survey frequently includes conventional radiography (CR) and CT scans of the extremities if deemed necessary. This approach is advocated in order to minimise the risk of missing a clinically important injury or unstable spinal fractures that can potentially lead to serious impairment $[9,10]$ and legal ramifications $[11,12]$.

Since high image quality of CT scans is a prerequisite for diagnostic workup, any disturbances, such as artefacts or noise, should be kept to a minimum. Artefacts can be limited by avoiding or removing objects with sharp transitions in material density [1316], while noise reduction can be achieved by increasing the radiation dose. However, exposure to ionising radiation is associated with increased cancer risk [17-20]. Several studies have indicated that $\mathrm{CT}$, but to a lesser degree also diagnostic conventional radiography (computed radiography; $\mathrm{CR}$ ) may account for a non-negligible increased risk of cancer $[17,19,21]$. It is therefore important that the benefits of (increased) radiation exposure should be weighed critically against its risks. In accordance with the ALARA (as low as reasonably achievable) principle in radiography [22], the materials placed between the $x$-ray source and $x$-ray detector should have a low level of absorption in order to minimise the radiation dose the patient is exposed to. In trauma workup, the level of radiation absorption by the spinal immobilisation device may vary significantly [23], thereby altering the radiation dose necessary to deliver high quality images. In this study, we compared devices for spinal stabilisation in terms of radiation transmission and the effect on objective image quality. The hypothesis in this study was that the devices would differ in the radiation dose necessary for good quality images. Our aim was to find out which of these devices is the preferred choice for spinal stabilisation from a radiation exposure point of view. 


\section{Materials and methods}

\section{Materials and set-up}

Radiation transmission and image noise for $\mathrm{CR}$ and $\mathrm{CT}$ were compared using two spineboards (Millennia Backboard; Ferno-Washington Inc, Wilmington, $\mathrm{OH}$; and a prototype soft-layered long spineboard [24]) in combination with two different headblocks (soft foam headblocks: universal head immobiliser, Ferno-Washington Inc, Wilmington, $\mathrm{OH}$; and new-design headblocks: speedblocks, Laerdal Medical, Stavanger, Norway) or none, and a vacuum mattress (RedVac; Radstadt, Austria). This means that we measured eight different set-ups (Table 7.1). The study was approved by the Medical Research Ethics Committee of the Maastricht University Medical Centre and registered as ISRCTN 68626238.

Table 7.1. Set-ups used.

\begin{tabular}{llll}
\hline Spinal immobilisation & Head immobilisation & CR & CT \\
\hline None & None & $\mathrm{X}$ & $\mathrm{X}$ \\
Rigid & None & $\mathrm{X}$ & $\mathrm{X}$ \\
Rigid & Soft foam & & $\mathrm{X}$ \\
Rigid & New design & $\mathrm{X}$ \\
Soft-layered & None & $\mathrm{X}$ & $\mathrm{X}$ \\
Soft-layered & Soft foam & & $\mathrm{X}$ \\
Soft-layered & New design & $\mathrm{X}$ & $\mathrm{X}$ \\
Vacuum mattress & None & $\mathrm{X}$ & \\
\hline
\end{tabular}

CR images were obtained using an over-Table Philips Trauma Diagnost (Philips, Best, the Netherlands). Transmitted radiation was measured using a $30 \mathrm{~mL}$ ionisation chamber and a Capintec 192A electrometer (Capintec, Inc, Ramsey, NJ, USA). A 3 mm thick lead shield with a $76 \times 45 \mathrm{~mm}$ window was placed between the device and the ionisation chamber to eliminate scattered radiation as much as possible. The set-up was rebuilt after each image had been taken, to mimic the variation in practical use of the devices. Each set-up was measured five times and with four different technique settings of the equipment, not using the automatic exposure control (AEC) (Table 7.2).

Table 7.2. Settings used

\begin{tabular}{llll}
\hline Setting & Tube potential $(\mathbf{k V})$ & Exposure time $(\mathbf{m s})$ & Tube current time (mAs) \\
\hline CR setting 1 & 60 & 165 & 100 \\
CR setting 2 & 75 & 157 & 100 \\
CR setting 3 & 100 & 220 & 100 \\
CR setting 4 & 120 & 271 & 100 \\
CT settings* & 120 & n.a. & 179 \\
\hline
\end{tabular}

* axial head scan, single rotation; collimation $64 \times 0.9 \mathrm{~mm}$

n.a.: not applicable 
CT images were obtained using a dual-source CT scanner (Definition Flash, Siemens Healthcare, Forchheim, Germany). A standard $16 \mathrm{~cm}$ diameter computed tomography dose index (CTDI) head phantom (Leeds Test Objects LTD, North Yorkshire, United Kingdom) was used in combination with a pencil ionisation chamber (Unfors RaySafe; Billdall, Sweden). The ionisation chamber was consecutively inserted in each of the five available holes of the phantom, with the other holes filled up with rods made of the same material as the phantom (Figure 7.1). The CTDI was subsequently calculated as $(1 / 3 *$ radiation dose measurement of central hole $)+(2 / 3 *$ average of the radiation dose measurements of peripheral holes) [25]. The phantom was placed on each of the devices, and secured in place using either headblocks or foam spacers (for the spineboards), or by modelling the vacuum mattress around the phantom. This procedure was repeated five times per set-up, thus mimicking variations in realistic placement of both the device and the head.

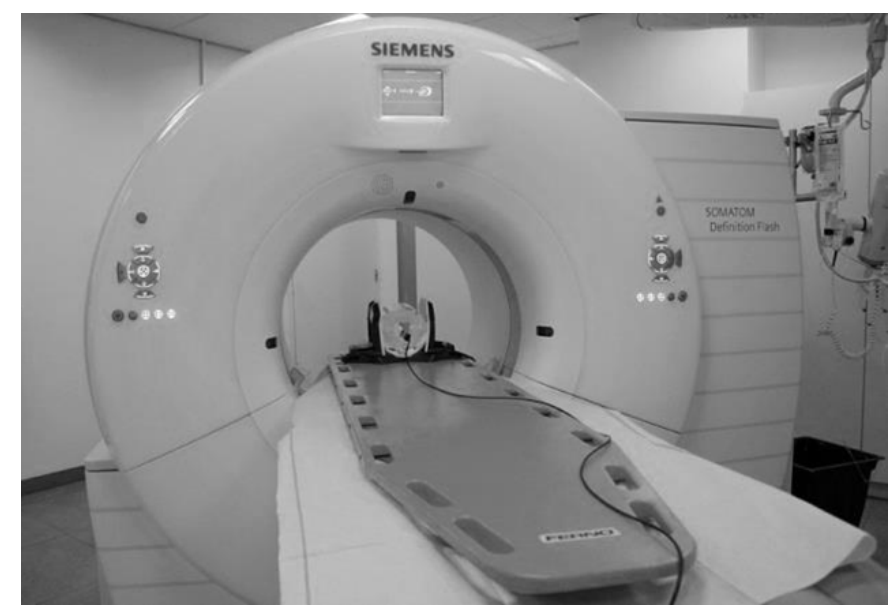

Figure 7.1. Set up example in CT: rigid spineboard plus new design headblocks.

\section{Calculations}

Transmission, i.e. the percentage of radiation that passed through the device, was calculated as the radiation dose passing with the device in place, divided by the radiation dose without the device in place. For $\mathrm{CR}$, the measured air kerma was taken as the radiation dose, while the CTDI-value was used for CT.

Image noise in CT was used as a measure of objective image quality and was defined as the standard deviation (SD) of the CT values (in Hounsfield Units) of the pixels within 16 circular regions of interest covering 51\% of the phantom (Figure 7.2). Image noise was determined for the middle slice of all phantom scans using ImageJ version 1.47v for Windows software (http://imagej.nih.gov/ij/). 


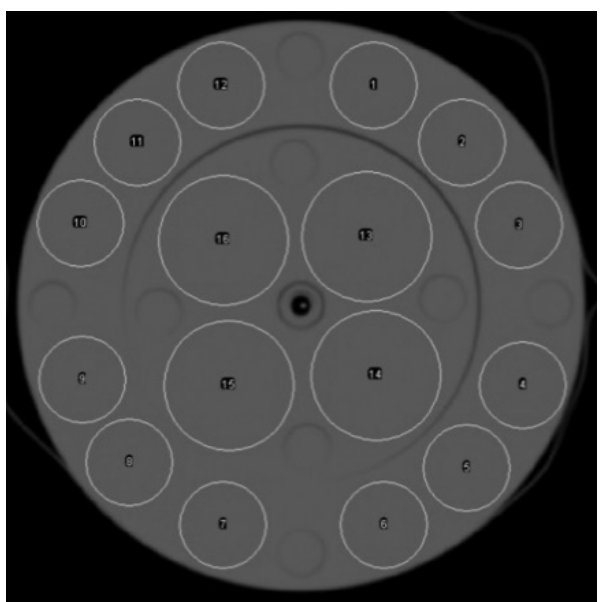

Figure 7.2. Regions of interest for noise measurements in CT

\section{Statistical analysis}

Statistical analyses were performed using SPSS (IBM, Chicago, IL), version 20.0.0. Data are presented as mean \pm SD. Non-parametric data were compared using Kruskal-Wallis testing for overall differences, and Mann-Whitney $U$ test for differences between devices. Parametric data were compared using repeated measure ANOVA for differences between devices. Significance was assumed at $P<0.05$.

\section{Results}

In CR, there was a significant main effect of device on transmission, $F(2,8)=163,914$ ( $P$ $<0.01)$, and a main effect of voltage on transmission, $F(3,12)=13,030(P<0.01)$ (Figure 7.3). Subsequent contrast tests revealed that transmission was highest for the vacuum mattress, followed by the rigid spineboard, and lowest for the soft-layered spineboard. These results were seen at all voltages. A significant interaction effect indicated that the differences between the devices became more pronounced with increasing voltages $F(6,24)=4,787(P<0.01)$. In $C T$, transmission was significantly lower when headblocks were used compared to no headblocks (Figure 7.4). Kruskal-Wallis testing for non-parametric groups showed an overall significant difference between the conditions $(P<0.001)$. 


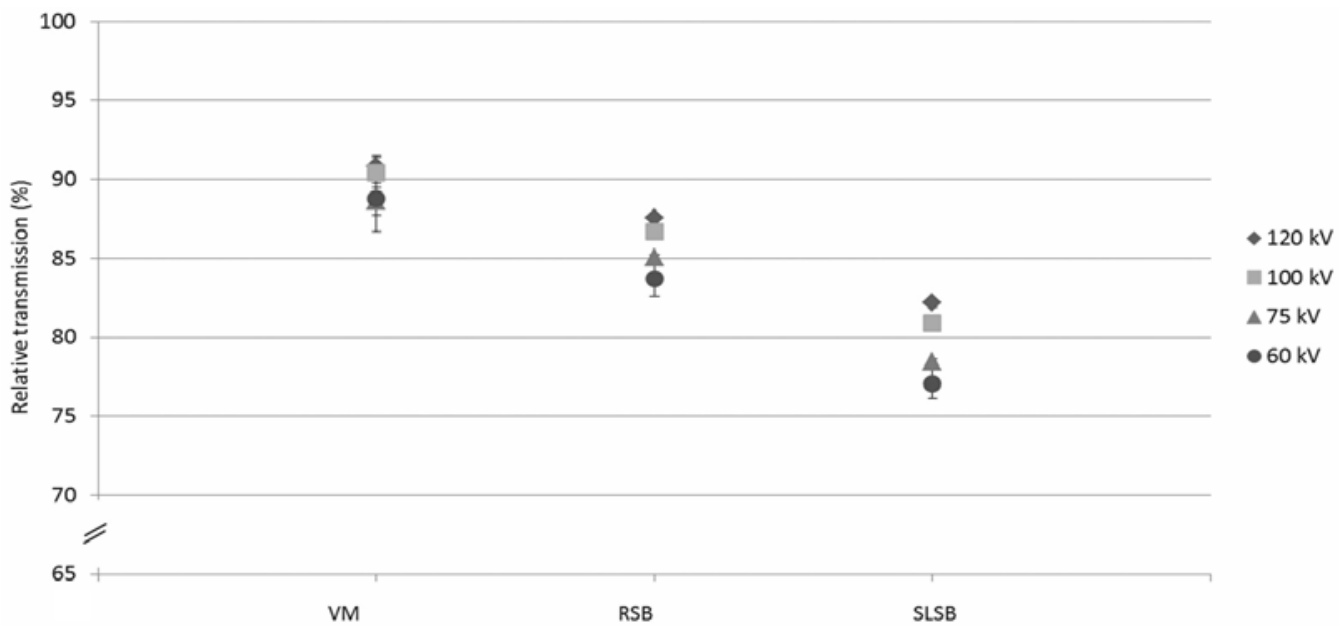

Figure 7.3. Relative transmission in CR for various devices* and different tube settings, relative to no device Presented data are means \pm SD

*VM: vacuum mattress; RSB: rigid spineboard; SLSB: soft-layered spineboard

For all tube voltages, Mann-Whitney tests showed significant differences after Bonferroni correction between all three devices at $P<0.0083$.

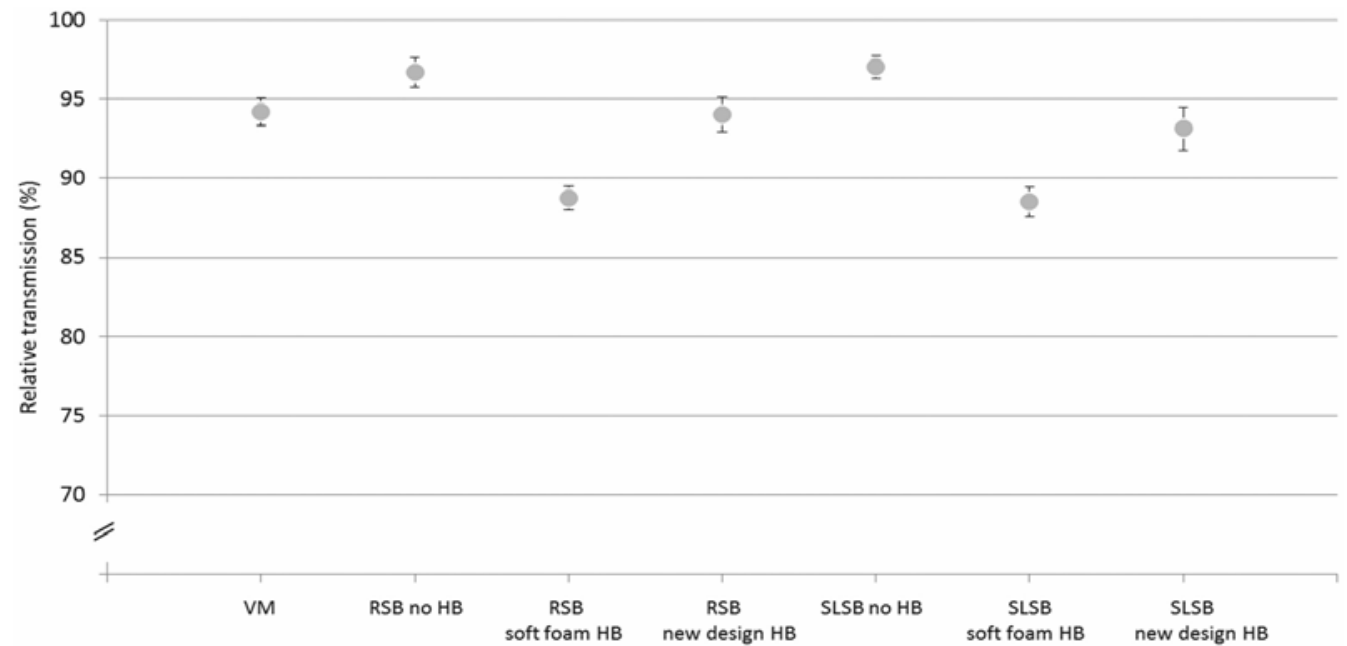

Figure 7.4. Relative transmission in CT for various devices*, relative to no device Presented data are means \pm SD

*VM: vacuum mattress; RSB: rigid spineboard; SLSB: soft-layered spineboard; HB: headblocks

- Significant difference $(P<0.05)$ for no device vs. vacuum mattress, RSB new design headblocks, rigid spineboard, SLSB new design headblocks, RSB soft foam headblocks, SLSB soft foam-layered spineboard, HB: headblocks

- Significant difference $(P<0.05)$ for vacuum mattress vs. SLSB soft foam headblocks

- Significant difference $(P<0.05)$ for SLSB no headblocks vs. SLSB soft foam headblocks 
With regard to noise in $\mathrm{CT}$, repeated measures ANOVA showed significant differences in noise between all conditions, $F(7,14)=60.9, P<0.01$ (Figure 7.5). As expected, a significant relationship was found between decreased transmission and increased noise in $\mathrm{CT}, \mathrm{r}=0.67, P<0.01$.

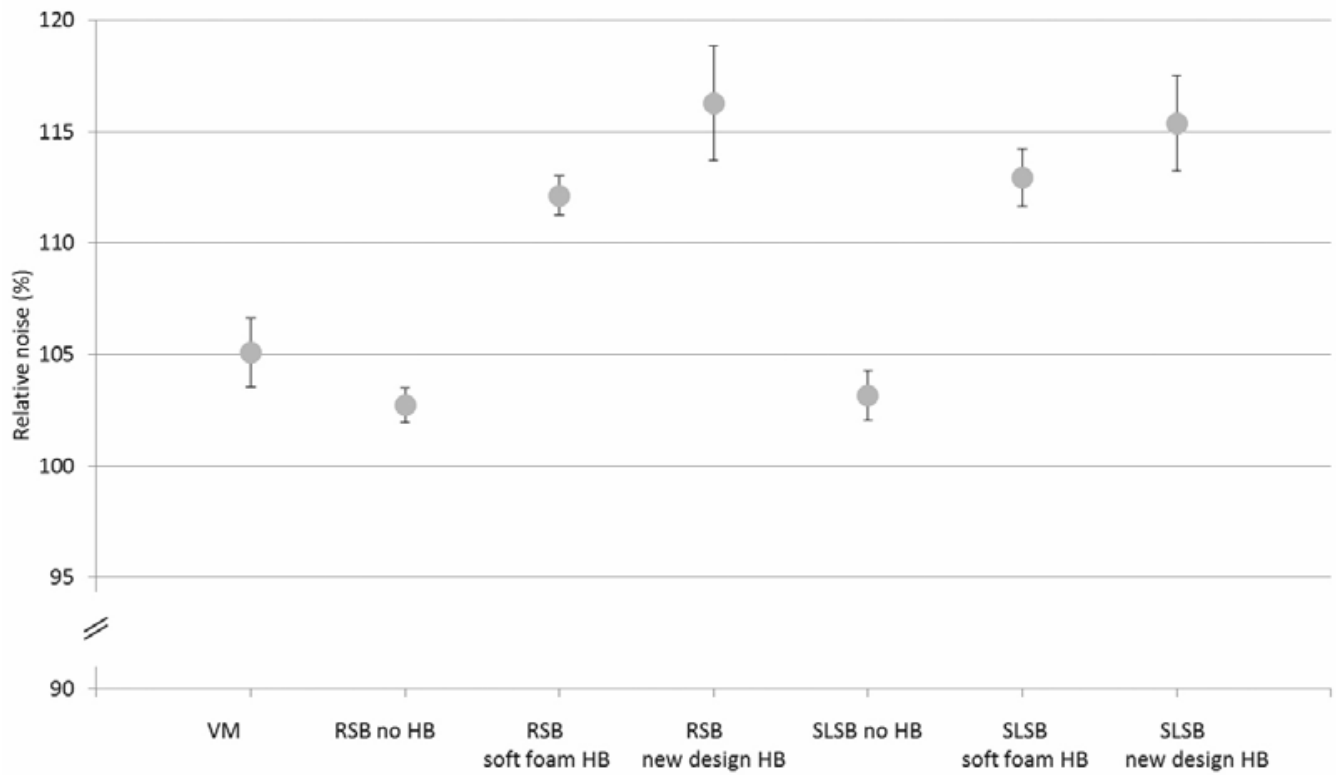

Figure 7.5. Relative noise in CT for various devices*, relative to no device Presented data are means \pm SD

*VM: vacuum mattress; RSB: rigid spineboard; SLSB: soft-layered spineboard; HB: headblocks

- Significant difference** $(P<0.05)$ for no device vs. RSB new design headblocks, SLSB no headblocks, SLSB soft foam headblocks, and SLSB new design headblocks

- Significant difference** $(P<0.05)$ for vacuum mattress vs. RSB soft foam headblocks, RSB new design headblocks, SLSB soft foam headblocks, and SLSB new design headblocks

- Significant difference** $(P<0.05)$ for RSB no headblocks vs. RSB soft foam headblocks, RSB new design headblocks, SLSB soft foam headblocks, and SLSB new design headblocks

- Significant difference** $(P<0.05)$ for RSB soft foam headblocks vs. RSB new design headblocks, SLSB no headblocks, and SLSB new design headblocks

- Significant difference** $(P<0.05)$ for RSB new design headblocks vs. SLSB no headblocks, and SLSB soft foam headblocks

- Significant difference** $(P<0.05)$ for SLSB no headblocks vs. SLSB soft foam headblocks, and SLSB new design headblocks

** significant differences after Bonferroni correction 


\section{Discussion}

This study compared a number of devices for spinal immobilisation with regard to radiation transmission and objective image quality. Although adding a spineboard or vacuum mattress to the set-up decreased both transmission and image quality, adding headblocks to the spineboards decreased transmission and image quality even more.

Despite radiation exposure in acute (trauma) care being an important issue [2629], studies examining radiation transmission in spineboards and vacuum mattresses have been sparse [23]. Our study adds to this knowledge of the radiographic properties of spineboards and vacuum mattresses, by including data regarding headblocks, which are considered an integral part of the equipment used to achieve full spinal immobilisation [30]. Current trauma protocols require the use of these devices, which absorb part of the radiation. CR and CT machines automatically adjust radiation dose to compensate for image quality loss due to absorption, but since any increase in radiation exposure creates an additional cancer risk [20, 21, 31, 32], it is important that the devices have high transmission rates.

Our results show that in $\mathrm{CR}$, transmission decreased by $9 \%$ to $23 \%$, depending on the device and tube voltage used. In CT, the transmission decreased by up to $11 \%$. When x-rays encounter any form of matter, they are partly transmitted and partly absorbed and scattered. The resulting transmission depends on the energy-dependent attenuation coefficient of the material and the x-ray energy (here determined by the system's tube voltage). The attenuation coefficient increases when the x-ray energy decreases. Consequently, it is to be expected that the transmission will be reduced when the tube voltage is lowered, as we have shown in Figure 7.3. The observed differences in transmission between the setups (Figures 7.3 and 7.4) are related to differences in material properties (i.e. attenuation coefficients) as well as differences in the amount of material present (compare the setup with and without headblocks present).

For all devices, transmission was higher for CT than for CR. This can be explained by considering that, in contrast to $\mathrm{CR}$, only some of the projections acquired in a CT scan involve $x$-ray trajectories through the device. In addition, the use of a thicker beam filter in $\mathrm{CT}$ results in more penetrating radiation compared to $\mathrm{CR}$, even at the same tube voltage. As trauma CRs are usually taken in anterior-posterior (AP) direction, the patient is actually being exposed to additional radiation dose, because the posteriorly placed immobilisation device absorbs part of the radiation that has already passed through the patient. However, it should be noted that although the relative extra radiation exposure is higher for $\mathrm{CR}$ than $\mathrm{CT}$, the absolute actual dose increase is in the order of 5 times higher for $\mathrm{CT}$, as the $\mathrm{CT}$ radiation exposure is higher to start with. Regardless of this, with ever more institutions moving away from CR and 
using CT instead [33], radiation protection according to ALARA principle should always be a priority. Our study shows that one simple way to reduce radiation exposure is to remove the headblocks before CT imaging. Nonetheless, in case of restless or nonresponsive trauma patients at risk for spinal injury, physicians are reluctant to remove the immobilisation devices before imaging has been completed, for fear of worsening or missing an injury [34] and possible consequent medical litigation [12]. However, no studies have so far been published showing a direct causal link between the use of immobilisation devices (or lack thereof) and injury outcome [35]. There are, however, sound arguments to permit removal of the devices in calm, cooperative patients [36].

Since increased exposure to ionisation radiation induces health risks [31, 32], even though the risks are small and consequently associated with large uncertainties [37], any measure to reduce radiation should be welcomed. A total-body trauma CT scan without immobilisation devices exposes the patient to $15-30 \mathrm{mSv}$ [38-40]. When this scan is performed on a rigid spineboard with soft foam headblocks in place, an increase in radiation exposure of $11 \%$ is needed to maintain image quality, increasing the radiation dose by 1.7-3.4 $\mathrm{mSv}$. Data from earlier studies on the risks of radiation exposure $[31,32]$ indicate that the additional risk of dying from cancer is $0.5 \%$ per 100 $\mathrm{mSv}$, assuming an estimated overall lifetime cancer risk of $25 \%$. Although the added risk of fatal cancer due to the use of devices for spinal immobilisation may be small, physicians should make an informed decision not only on whether or not to make use CR or CT in on an immobilised patient, but also on the question whether some of the devices can be removed before imaging, factoring in both the radiation risks and the risks of potentially missed injuries. Furthermore, designers of devices for spinal immobilisation should take into account the radiological properties of their devices.

Some remarks can be made with regard to our study. First, we only looked at noise as an indicator of image quality. We did not look at other factors, such as artefacts (beam hardening, additional scattering and banding) caused by differences in attenuation by the devices. This is becoming increasingly important, as there is a trend towards lower dosages, which in turn may result in more visible artefacts. This subject is discussed in detail elsewhere [13]. Second, we evaluated only one CT scanning protocol using one multidetector CT scanner. We chose to consider only a tube voltage of $120 \mathrm{kV}$, as this is the clinical standard for trauma diagnostics in our hospital. Although beam filtration may be different for CT scanners of other manufacturers, resulting in somewhat different beam qualities, we believe that the results will be qualitatively comparable. 


\section{Conclusion}

There are significant differences in radiation transmission depending on the type of immobilisation device used. In $\mathrm{CR}$, the vacuum mattress showed the highest transmission rates, while in $\mathrm{CT}$, the spineboards without headblocks performed best in terms of both transmission and noise. In view of the duty to keep patient radiation exposure as low as possible while still achieving the required image quality, the results of this study can help in making an informed decision whether the risks associated with radiation exposure outweigh those of missed injuries. 


\section{References}

1. Farrington JD. Extrication of victims - surgical principles. J Trauma. 1968. 8(4):493-512.

2. Farrington JD. Death in a ditch. Bull AM Coll Sur. 1967. 52:121-130.

3. Cooke MW. Use of the spinal board within the accident and emergency department. J Accid Emerg Med. 1998. 15:108-113.

4. Lerner EB \& Moscati R. Duration of patient immobilization in the ED. Am J Emerg Med. 2000. 18:2830.

5. Malik MHA \& Lovell ME. Current spinal board usage in emergency departments across the UK. Injury. 2003. 34:327-329.

6. Stagg MJ \& Lovell ME. A repeat audit of spinal board usage in the emergency department. Injury. 2008. 39:323-326.

7. El-Khoury GY, Kathol MH \& Daniel WW. Imaging of acute injuries of the cervical spine: value of plain radiography, CT, and MR Imaging. Am J Roentgenol. 1995. 164:43-50.

8. France JC, Bono CM \& Vaccaro AR. Initial radiographic evaluation of the spine after trauma: when, what, where, and how to image the acutely traumatized spine. J Orthop Trauma. 2005. 19:640-649.

9. Hadida C \& Lamire J. Missed upper cervical spine fracture: clinical and radiological considerations. J Can Chiropr Assoc. 1997. 41:77-85.

10. Meek S. Fractures of the thoracolumbar spine in major trauma patients.British Med J. 1998. 317:14421443.

11. Berlin L. CT versus radiography for initial evaluation of cervical spine trauma: what is the standard of care? Am J Roentgenol. 2003. 180:911-915.

12. Lekovic GP. Litigation of missed cervical spine injuries in patients presenting with blunt traumatic injury. Neurosurgery. 2007. 60:516-523.

13. Hemmes B, Jeukens CRLPN, Al-Haidaari A, et al. Effect of spineboard and headblocks on the image quality of head CT scans. Submitted.

14. Miller JA, Mele C \& Abu-Judeh H. Significance of backboard artifacts on portable trauma series chest radiographs. Emerg Radiol. 1999. 6:334-338.

15. Daffner RH \& Khoury MB. Pseudofractures due to Nec-Loc cervical immobilization collar. Skeletal Radiol. 1987. 16:460-462.

16. Schou J, Kiermayer H, Ummenhofer W \& Herion H-P. In search of the most suitable technique for truncal spinal immobilization with associated radiography. Eur J Emerg Med. 2001. 8:89-92.

17. Berrington de Gonzalez A \& Darby S. Risk of cancer from diagnostic x-rays: estimates for the UK and 14 other countries. Lancet. 2004. 363:345-351.

18. Shimizu Y, Schull WJ \& Kato H. Cancer risk among atomic bomb survivors. The RERF Life Span Study. JAMA, 1990. 264(5):601-604.

19. Einstein AJ, Henzlova MJ \& Rajagopalan S. Estimating Risk of Cancer Associated With Radiation Exposure From 64-Slice Computed Tomography Coronary Angiography. JAMA, 2007. 298:317-323.

20. Hall EJ \& Brenner DJ. Cancer risks from diagnostic radiology. British J Radiol. 2008. 81:362-378.

21. Levy AR, Goldberg MS, Hanley JA, Mayo NE \& Benoit P. Projecting the lifetime risk of cancer from exposure to diagnostic ionising radiation for adolescent idiopathic scoliosis. Health Physics. 1994. 66:621-633.

22. International Commission on Radiological Protection, Radiation protection. Annals of the ICRP, Publication 26 (New York: Pergamon Press), 1977.

23. Linsenmaier $U$, Krotz M, Kanz K-G et al. Evaluation von Wirbelsaulen-brettern fur die Roentgendiagnostik. Fortschr Rontgenstr. 2001. 173:1041-1047.

24. Hemmes B, Poeze M \& Brink PRG. Reduced tissue-interface pressure and increased comfort on a newly developed soft-layered long spineboard. J Trauma. 2010. 68:593-598. 
25. Menzel, H, Schibilla H, \& Teunen D. European guidelines on quality criteria for computed tomography. Luxembourg: European Commission 16262; 2000.

26. Bregstein JS, Lubell TR, Ruscica AM \& Roskind CG. Nuking the radiation: minimizing radiation exposure in the evaluation of pediatric blunt trauma. Curr Opin Pediatr. 2014. 26:272-278.

27. Kruger JF, Chen AH, Rybkin A, Leeds K, Frosch DL \& Goldman LE. Clinician perspectives on considering radiation exposure to patients when ordering imaging tests: a qualitative study. BMJ Qual Saf, 2014.

28. Corwin MT, Sheen L, Kuramoto A, Lamba R, Parthasarathy S \& Holmes JF. Utilization of a clinical prediction rule for abdominal-pelvic CT scans in patients with blunt abdominal trauma. Emerg Radiol. 2014. 21:571-576.

29. Mills AM, Raja AS \& Marin JR. Optimizing Diagnostic Imaging in the Emergency Department. Acad Emerg Med. 2015. 22:625-631.

30. American College of Surgeons, ed. Advanced Trauma Life Support. Student course manual, $9^{\text {th }}$ ed. 2012: Chicago, IL

31. International Commission on Radiological Protection, The 2007 recommendations of the International Commission on Radiological Protection. Annals of the ICRP; 2007. 37:1-332.

32. National Research Council, Health risks from exposure to low levels of ionizing radiation: BEIR VII Phase 22006, Washington DC: National Academic Press.

33. Heller MT, Kanal E, Almusa O, et al. Utility of additional CT examinations driven by completion of a standard trauma imaging protocol in patients transferred for minor trauma. Emerg Radiol., 2014. 21: 341-347.

34. Morrissey JF, Kusel ER, \& Sporer KA. Spinal motion restriction: an educational and implementation program to redefine prehospital spinal assessment and care. Prehosp Emerg Care. 2014.18:429-432.

35. Kwan I, Bunn F \& Roberts IG. Spinal immobilisation for trauma patients (review). Cochrane database of systematic reviews, 2009

36. Hauswald M. A re-conceptualisation of acute spinal care. Emerg Med J. 2013. 30:720-723.

37. Hendee WR \& O'Connor MK. Radiation risks of medical imaging: separating fact from fantasy. Radiology. 2012. 264:312-321.

38. Brenner DJ, Doll R, Goodhead DT et al, Cancer risk attributable to low doses of ionizing radiation: Assessing what we already know. Proc Natl Acad Sci USA. 2003. 100:13761-13766.

39. Sierink JC, Saltzherr TP, Wirtz MR, Streekstra GJ, Beenen LFM \& Goslings JC. Radiation exposure before and after the introduction of a dedicated total-body CT protocol in multitrauma patients. Emerg Radiol. 2013. 20:507-512.

40. Mettler FA, Huda W, Yoshizumi TT \& Mahesh M. Effective doses in radiology and diagnostic nuclear medicine: a catalog. Radiology. 2008. 248:254-263. 



\section{Summary and conclusions}

Samenvatting en conclusies
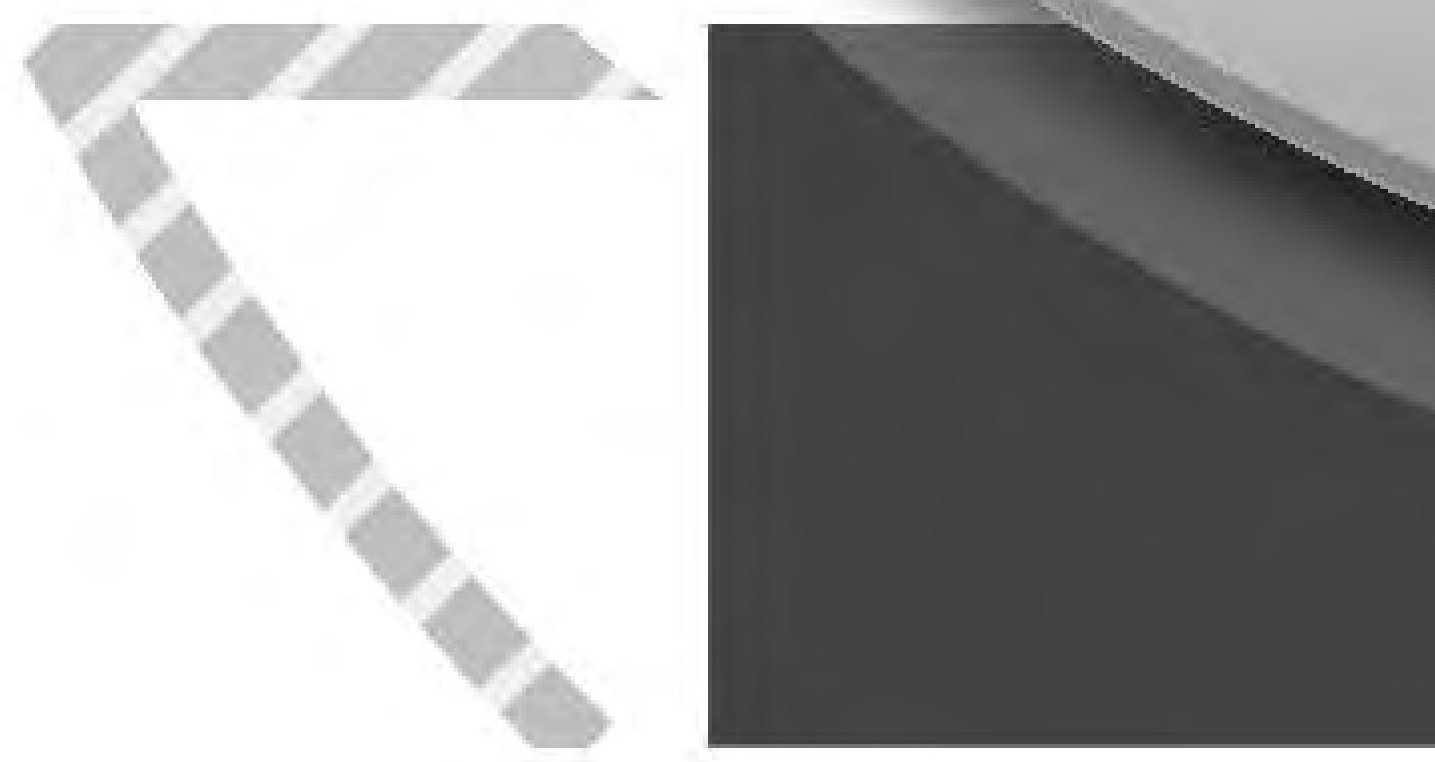

Baukje Hemmes 


\section{Summary and conclusions}

The currently used rigid spineboard was introduced as a prehospital extrication and transport device in the 1930s [1]. A number of publications in the subsequent decades [2-5] led to the generally accepted paradigm that trauma patients at risk of spinal injury should be immobilised on a spineboard in order to prevent worsening of the injury $[6,7]$. However, since the late 1980 s attention has been drawn to the adverse effects of using a rigid spineboard, including breathing difficulties [8-10], increased intracranial pressure in patients with brain injury [11-13], pain caused by lying on the spineboard [14-16] and the development of pressure ulcers [17, 18]. In addition, no study so far has been able to provide evidence that using a spineboard prevents further injury to the already damaged spine [19]. Consequently, the paradigm for using the spineboard gradually shifted from indiscriminate use of the rigid spineboard to both a more differentiated used $[20,21]$ and the use of alternatives, such as the vacuum mattress. Nevertheless, current protocols still recommend its use [6, 7, 22]. Since all methods for spinal immobilisation have their advantages and disadvantages, there was a need to develop and test a new device that has the stability of the spineboard when used as an extrication device, with as few disadvantages as possible when used during transport. To meet this need, we developed a soft-layered spineboard, consisting of a rigid plastic shell with a built-in mattress, covered with a plastic sheet. In the current thesis, a number of studies are presented which compare the properties of the new soft-layered spineboard with those of the rigid spineboard and the vacuum mattress. The studies discussed in the various chapters focused on pressure relief and comfort, tissue deformation, biochemical markers of tissue damage and radiologic considerations.

\section{Pressure relief and comfort}

Two studies focused on the clinical evaluation of the tissue-interface pressure. The main goal of the use of a tissue-protective device such as a mattress is to decrease tissue-interface pressures by providing pressure relief. This is a clinically important issue because prolonged pressures exceeding the local blood pressure in arterioles or capillaries can obstruct blood flow in the soft tissues, eventually leading to tissue ischemia [23-25]. This in turn can lead to pressure ulcers, which can be extremely painful [26-28] and debilitating [29,30] and lead to a sharp rise in medical costs due to prolonged hospitalisation [31, 32]. Although the exact threshold for tissue ischemia is unknown, it is clear that there is a trade-off between pressure and time, with higher pressures resulting in tissue damage sooner than lower pressures [24, 33, 34]

In the study reported on in Chapter 2, healthy awake volunteers were placed on a rigid spineboard, a vacuum mattress and a soft-layered spineboard for 15 minutes 
each, during which periods tissue-interface pressures were measured using a pressuremapping device, and comfort was scored at the beginning and end of this period on a 10-point VAS scale. Results of this study showed that the rigid spineboard and vacuum mattress induced high tissue-interface pressures at the sacrum, shoulders and heels. Using the soft-layered spineboard led to a significant reduction in both tissue-interface pressure and discomfort compared to the other devices. We found that discomfort increased over time for all three devices, even though the tissue-interface pressures remained stable throughout the 15-minute test period. Whereas this study already found high tissue-interface pressures and discomfort during the limited observational period of 15 minutes, in practice the time spent on the spineboard can easily be longer $[35,36]$. The discomfort induced may lead to or increase restlessness due to the urge to relieve the pain, and may prompt physicians to increase the number of unnecessary diagnostic procedures. A recent patient survey at the Maastricht University Medical Centre emergency department (unpublished data) indicated that for the vast majority of patients, the level of discomfort was significantly reduced when they were transferred from the rigid spineboard onto a standard hospital bed or a soft-layered spineboard.

The study discussed in Chapter $\mathbf{3}$ evaluated tissue-interface pressures in patients unable to alleviate the discomfort of lying on the rigid spineboard. A group of anaesthetised patients was used as a proxy for unconscious or paralysed patients, who are almost without exception transported on a spinal immobilisation device. We hypothesised that anaesthetised patients would show different tissue-interface pressure patterns compared to a group of awake volunteers. Patients in this study were randomised to either the rigid or the soft-layered spineboard, on which they remained for the entire surgical procedure. Tissue-interface pressures were monitored, and the appearance of the skin, such as redness resulting from the (relative) ischemia-reperfusion period was evaluated. Maximum time on the spineboards was limited to two hours. Contrary to our expectations, tissue-interface pressure distribution patterns in anaesthetised patients were comparable to those of the awake volunteers for both devices. The extent of redness was significantly lower for the soft-layered spineboard compared to the rigid spineboard, corresponding to significantly lower tissue-interface pressures.

In conclusion, these two studies showed that, compared to a rigid spineboard and vacuum mattress, the use of a soft-layered spineboard reduces the tissue-interface pressures, with less discomfort than on the rigid spineboard and vacuum mattress. 


\section{Tissue deformation}

Although tissue-interface pressures, redness of the skin and discomfort can be used as indicators of tissue pressure-related perfusion problems, measures of tissue deformation are also important indicators of the effects of these pressures. Chapter 4 reports on a study evaluating tissue deformation in healthy volunteers using MRI scans of the buttocks and a finite elements (FE) model to compute the tissue strains. The FE model was built for both the unloaded and loaded conditions for subjects with a normal, low and high BMI. This study showed that tissue deformation on the rigid spineboard was significantly higher than on the soft-layered spineboard. The high-BMI subject showed considerably higher strains and stresses than the low-BMI subjects. This implies that high-BMI patients are at greater risk for tissue ischemia due to the pressure caused by their body weight, especially when placed on hard surfaces such as a rigid spineboard.

Pressure ulcers are hypothesised to develop either from the skin surface and expand inwards [37], or from the bone-soft tissue boundary and expand outwards to the skin, the latter being known as deep-tissue injury [38].The model for the high-BMI subject showed particularly high stresses at the bone-soft tissue boundary, in addition to high stresses at the skin. This phenomenon of deep tissue deformation was less pronounced in the normal BMI subject and the low-BMI subject, although still present in these groups.

Current risk scales are not sensitive in predicting pressure ulcers in patients [39, 40]. A prospective study to evaluate the predictive value of MRI scan-based FE models for pressure ulcer development could provide valuable insights into the relationship between tissue deformation and pressure ulcer development.

\section{Biomarkers for tissue damage}

Chapter $\mathbf{5}$ reports on a study into the effect of tissue-interface pressure on the biochemical response of the skin, as a more direct indicator of tissue damage. Among the group of biomarkers, cytokine IL1, which is released in reaction to internal and external stimuli to the skin, plays a key role in the initiation and development of inflammatory and immune responses [41]. In normal conditions, IL1 is stored in the skin and lost by desquamation of the stratum corneum. However, upon skin injury or even mechanical deformation, a large amount of stored IL-1 is released and can trigger a rapid immune response [41, 42]. In the human epidermis, the majority of IL-1 activity from keratinocytes involves IL1 $\alpha$.

Another important aspect is that pressure on soft tissues reduces blood flow. After the pressure is relieved, the body reacts by increasing blood flow to allow for faster removal of waste products and supply of oxygen and nutrients. This increased blood flow results in redness of the skin. 
Twelve healthy volunteers were assessed to determine tissue responses when lying on a rigid and a soft-layered spineboard for one hour. During this one hour loading, and the subsequent unloaded hour, special absorptive tapes (Sebutapes) were used to collect sebum of the skin overlying the sacrum. Results showed that IL1 $\alpha$ release strongly correlated with the duration of the tissue-interface pressure, but not with the magnitude of the pressure. IL1 $\alpha$ was related to the level of skin redness, regardless of the type of spineboard the volunteer had been lying on. These findings imply that in the prevention of soft tissue damage due to tissue-interface pressures, the focus should not only be on pressure magnitude but also on reducing the duration. In clinical practice, this means both using a softer alternative to the rigid spineboard and releasing the patient from the spineboard as soon as possible.

\section{Radiological properties}

Finally, we evaluated the radiological properties of the rigid spineboard, vacuum mattress and soft-layered spineboard. We first determined the amount of radiation absorbed by the different immobilisation devices. In general, absorption of radiation weakens the image quality. Therefore, an automatic exposure control (AEC) adjusts the radiation dose needed to obtain good quality images, thereby increasing the radiation dose received by the patient. Higher doses of radiation are linked to an increased lifetime risk of cancer [43-45] and should therefore be avoided. We used a CTDI phantom and electrometer to measure the radiation exposure. As described in Chapter 6, the vacuum mattress had higher transmission rates in conventional radiography compared to both the rigid and soft-layered spineboards, whereas in CT, both spineboards without headblocks outperformed the vacuum mattress. However, adding headblocks to the spineboard significantly worsened transmission. This can be explained by the fact that in CT scanning, images are made in a 360-degree procedure, in which the headblocks represent an additional obstruction in the path of the beams, resulting in lower transmission. In noise, a similar effect was seen regarding noise, with the headblocks adding significantly to the decrease in image quality.

The other radiological issue, addressed in Chapter 7, was the artefacts induced by spinal immobilisation devices. Artefacts are distortions of radiological images caused by any obstacle located between the radiation beam and the receiver. This can be the spineboard and headblocks, but also other sources such as dentures, jewellery and zippers. For this study we evaluated the artefacts on CT scans of the head using both a special head phantom and actual patients. Although a considerable number of artefacts were found in these scans, these were not due to the rigid or soft-layered spineboard itself, but mainly to the sharp corners of the headblocks, with the square Ferno universal headblocks resulting in line artefacts, while the curvy Laerdal Speedblocks caused more diffuse blurring. We also investigated whether these 
artefacts compromise the clinical judgement of possible injuries. Comparing comments from a junior and an experienced radiologist, we found that the junior radiologist regarded more artefacts induced by the spineboard and headblocks as hampering the radiological evaluation of the $\mathrm{CT}$ scan than the experienced radiologist. The junior radiologist felt insecure in declaring no injuries were present, whereas the senior felt confident declaring no injuries were present even in the presence of artefacts. Followup CT scans showed that no injuries had been missed. In four cases (2\%), however, artefacts hampered judgement to such a degree that pathology could not be excluded, although a follow-up CT scan (without immobilisation device) showed no sign of injury.

From a radiological point of view, therefore, CT scans of trauma patients should ideally be made without immobilisation devices in place. This requires the physician to remove the device before exclusion is given about the presence of the injury, which is at odds with current protocols for trauma care [6]. Making the spineboard of a radiotranslucent material would be the next best option.

\section{Final remarks}

Our studies show that the soft-layered spineboard is a good alternative to the rigid spineboard from the point of view of comfort, pressures and soft tissue deformation and -damage. However, even when using a soft-layered spineboard, care providers should remove the patient from the spineboard as soon as possible. 


\section{References}

1. Warden CA. Splint stretcher board, United States Patent Office; 1938: United States of America.

2. Thomson JEM. First aid and transportation of suspected spine injuries. Am J Surg. 1939. XLV: 42-47.

3. Moir JL. First-aid treatment for fractured spines. British Med J. 1942. Oct 31: 529.

4. Geisler WO, Wynne-Jones M \& Jousse AT. Early management of the patient with trauma to the spinal cord. Med Serv J Can. 1966. 22:512-523.

5. Farrington JD. Death in a ditch. Bull Am Coll Surg. 1967. 98(6):44-53.

6. American College of Surgeons, ed. Advanced Trauma Life Support. Student course manual, $9^{\text {th }}$ ed. 2012: Chicago, IL

7. National Association of Emergency Medical Technicians, ed. PHTLS: Prehospital Trauma Life Support. $8^{\text {th }}$ ed. 2014, Jones \& Bartlett Learning.

8. Ay D, Aktas C, Yesilyurt S, Sarikaya S, Cetin A, \& Ozdogan ES. Effects of spinal immobilization devices on pulmonary function in healthy volunteer individuals. Ulus Travma Acil Cerrahi Derg (Turkish Journal of Trauma and Emergency Surgery). 2011. 17:103-107.

9. Bauer D, \& Kowalski R. Effect of spinal immobilization devices on pulmonary function in the healthy, nonsmoking man. Ann Emerg Med. 1988. 17(9):915-918.

10. Walsh M, Grant T, \& Mickey S. Lung function compromised by spinal immobilization. Correspondence. Ann Emerg Med. 1990. 19:615-616.

11. Agbeko RS, Pearson SBS, Peters MJ, McNames J, \& Goldstein B. Intracranial pressure and cerebral perfusion pressure responses to head elevation changes in pediatric traumatic brain injury. Pediatr Crit Care Med. 2012. 13:e39-e47.

12. Meixensberger J, Baunach S, Amschler J, Dings J \& Roosen K. Influence of body position on tissue-pO2, cerebral perfusion pressure and intracranial pressure in patients with acute brain injury. Neurol Res. 1997. 19:249-253.

13. Ng I, Lim J \& Wong HB. Effects of head posture on cerebral hemodynamics; its influences on intracranial pressure, cerebral perfusion pressure and cerebral oxygenation. Neurosurgery. 2004. 54: 593-598.

14. Cordell WH, Hollingsworth JC, Olinger ML, Stroman SJ \& Nelson DR. Pain and tissue-interface pressures during spine-board immobilization. Ann Emerg Med. 1995. 26:31-36.

15. Cross DA \& Baskerville J. Comparison of perceived pain with different immobilization techniques. Prehosp Emerg Care. 2001. 5:270-274.

16. Luscombe MD \& Williams JL. Comparison of a long spinal board and vacuum mattress for spinal immobilisation. Emerg Med J. 2003. 20: 476-478.

17. Lovell ME \& Evans JH. A comparison of the spinal board and the vacuum stretcher, spinal stability and interface pressure. Injury. 1994. 25:179-180.

18. Schouten R, Albert T \& Kwon BK. The spine-injured patient: initial assessment and emergency treatment. J Am Acad Orthop Surg. 2012. 20:336-346.

19. Kwan I, Bunn F \& Roberts IG. Spinal immobilisation for trauma patients (review). Cochrane database of systematic reviews; 2009.

20. Hauswald M. A re-conceptualisation of acute spinal care. Emerg Med J. 2013. 30:720-723.

21. White CC, Domeier R, Millin MG and the Standards and Clinical Practice Committee, National Association of EMS Physicians. EMS spinal precautions and the use of the long backboard - resource document to the position statement of the national association of EMS physicians and the American College of Surgeons committee on trauma. Prehosp Emerg Care. 2014. 18:306-314.

22. Ambulancezorg Nederland. Landelijk Protocol Ambulancezorg (Dutch National Protocol on Ambulance Care) 2014. Zwolle, the Netherlands. 
23. Berg G, Nyberg S, Harrison P. Baumchen J, Gurss E \& Hennes E. Near-infrared spectroscopy measurement of sacral tissue oxygen saturation in healthy volunteers immobilized on rigid spine boards. Prehosp Emerg Care. 2010. 14:419-424.

24. DeFloor T. The risk of pressure sores: a conceptual scheme. J Clin Nurs. 1999. 8:206-216.

25. Mak AFT, Yu Y, Kwan LPC, Sun L \& Tam EWC. Deformation and reperfusion damages and their accumulation in subcutaneous tissues during loading and unloading: a theoretical modeling of deep tissue injuries. J Theoretical Biology. 2011. 11:65-73.

26. Gunes UY. A descriptive study of pressure ulcer pain. Ostomy Wound Manag. 2008. 54:56-61.

27. Pieper B, Langemo D \& Cuddigan J. Pressure ulcer pain: a systematic literature review and National Pressure Ulcer Advisory Panel white paper. Ostomy Wound Manag. 2009. 55:16-31.

28. Rastinehad D. Pressure ulcer pain. J Wound Ostomy Cont. 2006. 33:252-257.

29. Fox C. Living with a pressure ulcer: a descriptive study of patients' experiences. Br J Community Nurs. 2002. 7:10-22.

30. Hopkins A, Dealey C, Bale S, DeFloor T \& Worboys F. Patient stories of living with a pressure ulcer. J Adv Nurs. 2006. 56:345-353.

31. Allman RM, Damiano AM \& Strauss MJ. Pressure ulcers, hospital complications, and disease severity: impact on hospital costs and length of stay. Adv Skin Wound Care. 1999. 12:22-30.

32. Graves N, Birrell FA \&Whitby M. Modeling the economic losses from pressure ulcers among hospitalized patients in Australia. Wound Repair Regen. 2005. 13:465-467.

33. Stekelenburg A, Oomens WJ \& Bader DL. Compression-induced tissue damage: animal models, in Pressure ulcer research: current and future perspectives, Bader DL, Bouten CVC, Colin D \& Oomens CWJ. (Eds) 2005, Springer-Verlag.187-204.

34. Linder-Ganz E, Engelberg S, Scheinowitz M \& Gefen A. Pressure-time cell death threshold for albino rat skeletal muscles as related to pressure sore biomechanics. J Biomech. 2006. 39:2725-2732.

35. Bouland AJ, Jenkins JL, \& Levy MJ. Assessing attitudes toward spinal immobilization. J Emerg Med. 2013. 45:e117-e125.

36. Stagg MJ \& Lovell ME. A repeat audit of spinal board usage in the emergency department. Injury. 2008. 39:323-326.

37. Black J, Baharestani MM, Cuddigan J, et al. National Pressure Ulcers Advisory Panel's updated pressure ulcer staging system. Adv Skin Wound Care. 2007. 20:269-274.

38. European Pressure Ulcer Advisory Panel, National Pressure Ulcer Advisory Panel, Prevention and treatment of pressure ulcers: clinical practice guideline 2009, Washington DC.

39. Schoonhoven L, Haalboom JRE, Bousema MT, et al. Prospective cohort study of routine use of risk assessment scales for prediction of pressure ulcers.Brit Med J.. 2002. 325: 797-801.

40. Anthony D, Papanikolaou P, Parboteeah S \& Saleh M. Do risk assessment scales for pressure ulcers work? J Tissue Viabil. 2010. 19:132-136.

41. Uchi H, Terao H, Koga T \& Furue M. Cytokines and chemokines in the epidermis. J Dermatol Sci. 2000. 24:S29-S38.

42. Lee RT, Briggs WH, Cheng GC, Rossiter HB, Libby P \& Kupper T. Mechanical deformation promotes secretion of IL-1alpha and IL-1 receptor antagonist. J Immunol. 1997. 159:5084-5088.

43. Berrington de Gonzalez A, \& Darby S. Risk of cancer from diagnostic x-rays: estimates for the UK and 14 other countries. Lancet. 2004. 363:345-351.

44. Einstein AJ, Henzlova MJ \& Rajagopalan S. Estimating Risk of Cancer Associated With Radiation Exposure From 64-Slice Computed Tomography Coronary Angiography. JAMA. 2007. 298(3): 317-323.

45. Hall EJ \& Brenner DJ. Cancer risks from diagnostic radiology. British J Radiol. 2008. 81:362-378. 


\section{Samenvatting en conclusies}

De harde wervelplank is al sinds de jaren '30 van de vorige eeuw in gebruik als prehospitaal extricatie- en transportmiddel [1]. Een serie publicaties in de daarop volgende decennia [2-5] heeft ertoe geleid dat men het gebruik van de wervelplank voor patiënten met een verdenking op wervelletsel als gouden standaard is gaan beschouwen $[6,7,8]$, vanuit het idee dat hiermee verergering van het letsel van de wervelkolom voorkomen kan worden. Sinds het eind van de jaren ' 80 heeft men echter meer aandacht gekregen voor de nadelen van het gebruik van zo'n harde wervelplank. Zo kan de patiënt moeilijker ademhalen door het insnoeren op de wervelplank [9-11], zorgt het horizontaal liggen voor verhoogde hersendruk bij patiënten met hersenletsel [12-14], veroorzaakt de plank ongemak en pijn [15-17], en kan het liggen op de harde wervelplank het ontstaan van drukplekken (decubitus) in de hand werken $[18,19]$. Daarnaast is er tot op heden nog geen enkel onderzoek gepubliceerd waaruit blijkt dat het gebruik van een harde wervelplank ook daadwerkelijk bijdraagt aan de preventie van verergering van letsel aan de reeds beschadigde wervelkolom [20]. Dientengevolge is er een verandering aan het optreden van standaard naar meer weloverwogen gebruik van de wervelplank [21, 22], of van een alternatief zoals de vacuüm matras. Ondanks deze verschuiving in denkwijze over het gebruik van de harde wervelplank is het gebruik ervan nog steeds een belangrijk onderdeel van de huidige protocollen voor zorg aan letselpatiënten [6-8]. Er is dan ook behoefte aan de ontwikkeling en het testen van een nieuw soort plank, dat dezelfde voordelen biedt als de huidige harde wervelplank bij extricatie, maar niet de nadelen kent wanneer de plank gebruikt wordt bij transport. Hiertoe hebben we een plank met ingebouwd matras ontwikkeld. Deze nieuwe "zachte" wervelplank bestaat uit een platte bak met daarin een afgedekt matras. In dit proefschrift worden een aantal onderzoeken gepresenteerd waarin de eigenschappen van de zachte wervelplank worden vergeleken met die van de harde wervelplank en de vacuüm matras. In de volgende paragrafen wordt ingegaan op drukverlaging en comfort, weefselvervorming, biochemische markers voor weefselschade en radiologische eigenschappen.

\section{Drukverlaging en comfort}

In twee klinische onderzoeken is gekeken naar de interactie tussen de ondergrond (stabilisatie methode) en de persoon, met betrekking tot druk op het lichaam. Het belangrijkste doel van het gebruik van een weefsel-beschermende laag zoals in de nieuw ontwikkelde wervelplank, is het verlagen van de druk die de ondergrond op het lichaam uitoefent. Dit is belangrijk omdat aanhoudende druk hoger dan die in het vaatbed, de doorbloeding van de zachte weefsels kan belemmeren, waardoor uiteindelijk weefselsterfte kan optreden [23-25]. Weefselsterfte kan leiden tot druk- 
plekken, die extreem pijnlijk [26-28] en hinderlijk [29, 30] zijn, en leiden tot langere ziekenhuisopnamen met bijkomende hoge kosten [31, 32]. Hoewel de exacte drempel voor het ontstaan van weefselsterfte onbekend is, is wel duidelijk dat er een balans is tussen druk en tijd, waarbij hogere druk sneller tot weefselschade leidt dan lagere druk $[24,33,34]$.

In hoofdstuk 2 worden de resultaten gepresenteerd van een studie waarin gezonde wakkere vrijwilligers elk gedurende telkens 15 minuten op een harde wervelplank, een vacuüm matras en een zachte wervelplank hebben gelegen. In deze tijd werd gemeten hoeveel druk het materiaal op het lichaam uitoefende, en gaven de vrijwilligers op een 10-puntsschaal aan hoe comfortabel ze de ondergrond vonden. De resultaten toonden aan dat de harde wervelplank en de vacuüm matras hoge en vergelijkbare drukken uitoefenden op het sacrum, de schouders en de hielen. De zachte wervelplank leidde tot significant lagere drukken en beter comfort vergeleken met de harde wervelplank en de vacuüm matras. Daarnaast nam het discomfort toe naarmate de vrijwilligers langer op de wervelplank en vacuüm matras lagen, ook al bleef de druk op het lichaam gelijk in de tijd. Terwijl in dit onderzoek de drukken en comfort maar voor een periode van vijftien minuten in kaart zijn gebracht, kan de tijd die een patiënt op een wervelplank of vacuüm matras doorbrengt, in de praktijk makkelijk veel langer zijn $[35,36]$. Het ongemak van liggen op een wervelplank of vacuüm matras kan rusteloosheid veroorzaken of verergeren doordat de patiënt dit ongemak wil opheffen. Een recent onderzoek onder patiënten van het MUMC (ongepubliceerde gegevens) liet zien dat bij de meeste patienten het ongemak van liggen op een harde wervelplank aanzienlijk werd verminderd wanneer zij werden overgeplaatst naar een ziekenhuisbed of zachte wervelpank.

In hoofdstuk $\mathbf{3}$ is gekeken naar de druk die de wervelplanken en vacuüm matras op het lichaam uitoefenen bij patiënten die niet in staat waren het ongemak te verminderen door te gaan verliggen. Een groep patiënten die onder algehele anesthesie een ingreep ondergingen, is gebruikt als benadering van bewusteloze of verlamde patiënten, die vrijwel zonder uitzondering op een wervelplank of vacuüm matras vervoerd worden van de plaats van het ongeval naar het ziekenhuis. De hypothese was dat bij patiënten onder algehele anesthesie het patroon van druk dat de wervelplank uitoefent op het lichaam, anders zou zijn dan bij wakkere mensen. De patiënten werden willekeurig geloot om gedurende de gehele ingreep op de harde of de zachte wervelplank te liggen. Naast de druk op het lichaam, werd ook de conditie van de huid in de gaten gehouden. Na een periode van (relatief) verminderde doorbloeding, zal door reperfusie de huid roder tonen. De maximale tijd op de wervelplanken werd gesteld op twee uur. In tegenstelling tot de verwachtingen, toonde de druk op het lichaam een vergelijkbaar patroon vergeleken met de wakkere vrijwilligers. De roodheid van de huid was beduidend minder voor de zachte 
wervelplank dan voor de harde wervelplank, overeenkomstig de lagere druk bij de zachte wervelplank.

Samengevat tonen deze twee studies aan dat gebruik van een zachte wervelplank leidt tot minder druk op het lichaam in vergelijking met de vacuüm matras en de harde wervelplank, en beter comfort.

\section{Weefselvervorming}

Druk op de lichaamsweefsels, roodheid van de huid en discomfort kunnen gebruikt worden als indicatoren voor drukgerelateerde doorbloedingsproblemen. Indicatoren die kijken naar weefselvervorming zijn daarnaast belangrijk om het effect van die druk in beeld te brengen. Met behulp van MRI scans van het bekken/bil-gebied en een eindige elementen model is bij gezonde vrijwilligers het effect van liggen op een harde of zachte wervelplank op weefselvervorming beschreven in hoofdstuk 4. Het eindige elementen model was gebouwd voor zowel de onbelaste als de belaste situatie in vrijwilligers met een laag, gemiddeld en hoog BMI. Deze studie toonde aan dat er significant sterkere weefselvervorming optrad bij liggen op de harde wervelplank vergeleken met de zachte wervelplank. Daarnaast waren vervormingskrachten significant hoger bij het hoog-BMI model dan bij het laag-BMI model. Hieruit kan afgeleid worden dat mensen met een hoog BMI een hoger risico lopen op het ontwikkelen van drukplekken door het gewicht van hun lichaam, met name wanneer ze op een harde ondergrond liggen zoals een harde wervelplank.

Drukplekken ontstaan ofwel aan het huidoppervlak waarna ze naar binnen verder ontwikkelen [37], ofwel op de grens van bot en zachte weefsels waarna ze naar het huidoppervlak toe groeien, ook wel bekend als drukgerelateerde diepe weefsel schade [38]. Met name in het hoog-BMI model werden, naast de hoge krachten op de huid, hoge vervormingskrachten gezien op de grens van zacht weefsel en bot. Bij het gemiddeld-BMI model en het laag-BMI model waren deze krachten in de diepe weefsels veel minder uitgesproken.

De bestaande risicoschalen voor het ontstaan van drukplekken zijn niet erg betrouwbaar $[39,40]$. Een prospectieve studie waarbij de voorspellende waarde van op MRI scans gebaseerde eindige elementen modellen worden gebruikt als voorspeller voor het ontstaan van drukplekken, zou waardevolle nieuwe informatie opleveren over het verband tussen weefselvervorming en het ontstaan van drukplekken.

\section{Biochemische markers van weefselschade}

In hoofdstuk $\mathbf{5}$ is gekeken naar de gevolgen van druk op de biochemische respons van de huid, als meest directe indicator van weefselschade. Een veelbelovende groep van biomarkers is cytokines, die worden vrijgemaakt in reactie op interne of externe prikkeling van de huid. Binnen de cytokines speelt het soort IL1 een belangrijke rol in 
de opstart en het verdere verloop van ontstekings- en andere immuun reacties [41]. Onder normale omstandigheden wordt IL1 bewaard in de huid en gaat deze verloren in het normale huidverouderings-proces. Bij letsel aan de huid of zelfs bij vervorming van de huid wordt een grote hoeveelheid IL1 vrijgemaakt, waardoor een snelle immuunreactie in gang gezet kan worden $[41,42]$. In de menselijke huid komt IL1 vooral vrij in de vorm van IL1 $\alpha$.

Daarnaast leidt de druk tot een afname van de doorbloeding van weefsels. Als de druk wordt opgeheven, reageert het lichaam daarop met een toename in de doorbloeding, zodat afvalproducten sneller kunnen worden afgevoerd en voedingsstoffen en zuurstof sneller het weefsel kunnen bereiken. Deze verhoogde doorbloeding is zichtbaar als roodheid van de huid.

Bij twaalf gezonde vrijwilligers is de huidreactie bepaald wanneer zij een uur op zowel de harde als de zachte wervelplank lagen. Gedurende dit uur, en het uur daarop volgend waarop de huid onbelast bleef, is de huiduitscheiding bij het sacrum middels speciale tapes (Sebutapes) verzameld. Uit de resultaten bleek dat het vrijkomen van IL1a sterk gerelateerd was aan hoe lang de druk op het lichaam was uitgeoefend, maar niet aan hoe intens die druk was. Daarnaast was er een verband tussen de hoeveelheid vrijgekomen IL1a en de roodheid van de huid, onafhankelijk van op welk soort wervelplank de persoon had gelegen. Deze bevindingen tonen aan dat voor het voorkómen van weefselschade niet alleen de intensiteit van de druk verlaagd moet worden, maar dat de duur dat iemand aan de druk wordt blootgesteld ook tot een minimum beperkt moet worden. In de praktijk betekent dit dat het belangrijk is om enerzijds een alternatief te gebruiken voor de harde wervelplank, en anderzijds de patiënt zo snel mogelijk van de wervelplank af te halen.

\section{Radiologische eigenschappen}

Tot slot zijn de radiologische eigenschappen van de harde en de zachte wervelplank, beide met en zonder hoofdsteunen, en de vacuüm matras met elkaar vergeleken. Eerst is gekeken naar de hoeveelheid straling die door de harde en zachte wervelplank en de vacuüm matras wordt geabsorbeerd. Hoe meer straling er wordt geabsorbeerd, hoe slechter de kwaliteit van het resulterende beeld wordt. Moderne Röntgen apparatuur corrigeert hier automatisch voor, door een hogere dosis straling te geven ter compensatie van de geabsorbeerde straling. Het lichaam wordt echter ook aan die hogere dosis straling blootgesteld, wat geassocieerd is met een hoger risico op kanker [43-45]. Daarom is het belangrijk de stralingsdosis zo laag mogelijk te houden. Met een CTDI hoofd-fantoom en een elektrometer is de blootstelling gemeten. In hoofdstuk 6 is te lezen dat de vacuüm matras een betere stralendoorlaatbaarheid had dan beide typen wervelplank bij het maken van gewone röntgenfoto's. Bij de CT scans gaven de wervelplanken juist een beter resultaat. Het toevoegen van de hoofdsteunen zorgde er 
echter voor dat de stralendoorlaatbaarheid in belangrijke mate afnam. Dit is te verklaren doordat CT scans in een 360-graden beweging worden gemaakt. Hierbij vormen de hoofdblokken een extra obstakel dat straling absorbeert, met als gevolg een lagere doorlaatbaarheid. Voor de beeldkwaliteit geldt eenzelfde effect, waarbij het toevoegen van de hoofdblokken resulteert in een duidelijke afname van de beeldkwaliteit.

In hoofstuk 7 is gekeken naar artefacten die te zien zijn op CT scans veroorzaakt door de wervelplanken en vacuüm matras. Artefacten zijn verstoringen in het beeld, veroorzaakt door een object tussen de zender en ontvanger van de stralenbundel. Dit kan de wervelplank of hoofdblokken zijn, maar ook bijvoorbeeld een kunstgebit, sieraden en ritssluitingen. In deze studie hebben we gekeken naar artefacten bij gebruik van een fantoom en bij scans van patiënten. Hoewel er op de patiënten-scans veel artefacten zijn gezien, werden deze over het algemeen niet veroorzaakt door een wervelpank of vacuüm matras, maar juist door de hoeken van de hoofdsteunen. De vierkante Ferno hoofdsteunen gaven lijnartefacten, terwijl de rondere Laerdal hoofdsteunen voor meer diffuse artefacten zorgden. Ook is gekeken of deze artefacten het klinisch oordeel hinderden. Hiertoe werden de scans beoordeeld door een junior radioloog en een ervaren radioloog en bleek ervaring een belangrijke rol te spelen. De junior radioloog oordeelde vaker dan de ervaren radioloog dat de artefacten de diagnose hinderden. Bovendien oordeelde de ervaren radioloog vaker dat er geen letsels zichtbaar waren op de CT scan, ook als er wel artefacten zichtbaar waren. In vier gevallen (2\%) kon de radioloog een afwijking echter niet geheel uitsluiten door aanwezigheid van artefacten, en bleek op de follow-up scans geen afwijking te zien. Ondanks dit verschil werden in de patiënten-scans geen letsels gemist wanneer we de scans vergeleken met follow-up scans.

Vanuit radiologisch oogpunt zouden CT scans van letselpatiënten daarom bij voorkeur gemaakt moeten worden zonder wervelplank, vacuüm matras en hoofdblokken. Hiervoor moet de behandelaar de patiënt van de wervelplank of vacuüm matras afhalen voordat bekend is of de patient letsel aan de wervelkolom heeft, wat in strijd is met de huidig geldende protocollen. De immobilisatie hulpmiddelen maken van materiaal dat optimaal röntgendoorlaatbaar is, is daarom de volgende beste optie.

\section{Tot slot}

Deze studies tonen aan dat de zachte wervelplank een goed alternatief is voor de harde wervelplank wat betreft comfort, druk en weefselvervorming en -schade. Echter, zelfs wanneer een zachte wervelplank gebruikt wordt, moet de patiënt zo snel mogelijk van die plank verwijderd worden. 


\section{Literatuur}

1. Warden CA. Splint stretcher board, United States Patent Office; 1938: United States of America.

2. Farrington JD. Death in a ditch. Bull Am Coll Surg. 1967. 98(6): p. 44-53.

3. Geisler WO, Wynne-Jones M \& Jousse AT. Early management of the patient with trauma to the spinal cord. Med Serv J Can. 1966. 22: p. 512-523.

4. Moir JL. First-aid treatment for fractured spines. British Med J. 1942. Oct 31: p. 529.

5. Thomson JEM. First aid and transportation of suspected spine injuries. Am J Surg, 1939. XLV: p. 42-47.

6. American College of Surgeons, ed. Advanced Trauma Life Support. Student course manual, 9th ed. 2012: Chicago, IL

7. National Association of Emergency Medical Technicians, ed. PHTLS: Prehospital Trauma Life Support. 8th ed. 2014, Jones \& Bartlett Learning.

8. Ambulancezorg Nederland. Landelijk Protocol Ambulancezorg (Dutch National Protocol Ambulance care) 2014, Zwolle, the Netherlands: Ambulancezorg Nederland.

9. Ay D, Aktas C, Yesilyurt S, Sarikaya S, Cetin A, \& Ozdogan ES. Effects of spinal immobilization devices on pulmonary function in healthy volunteer individuals. Ulus Travma Acil Cerrahi Derg (Turkish Journal of Trauma and Emergency Surgery). 2011. 17: p. 103-107.

10. Bauer D, \& Kowalski R. Effect of spinal immobilization devices on pulmonary function in the healthy, nonsmoking man. Ann Emerg Med. 1988. 17(9): p. 915-918.

11. Walsh M, Grant T, \& Mickey S. Lung function compromised by spinal immobilization. Correspondence. Ann Emerg Med. 1990. 19: p. 615-616.

12. Agbeko RS, Pearson SBS, Peters MJ, McNames J, \& Goldstein B. Intracranial pressure and cerebral perfusion pressure responses to head elevation changes in pediatric traumatic brain injury. Pediatr Crit Care Med. 2012. 13: p. e39-e47.

13. Meixensberger J, Baunach S, Amschler J, Dings J \& Roosen K. Influence of body position on tissue-pO2, cerebral perfusion pressure and intracranial pressure in patients with acute brain injury. Neurol Res. 1997. 19: p. 249-253.

14. Ng I, Lim J \& Wong HB. Effects of head posture on cerebral hemodynamics; its influences on intracranial pressure, cerebral perfusion pressure and cerebral oxygenation. Neurosurgery. 2004. 54: p. 593-598.

15. Cordell WH, Hollingsworth JC, Olinger ML, Stroman SJ \& Nelson DR. Pain and tissue-interface pressures during spine-board immobilization. Ann Emerg Med. 1995. 26: p. 31-36.

16. Cross DA, \& Baskerville J. Comparison of perceived pain with differenct immobilization techniques. Prehosp Emerg Care. 2001. 5: p. 270-274.

17. Luscombe MD, \& Williams JL. Comparison of a long spinal board and vacuum mattress for spinal immobilisation. Emerg Med J. 2003. 20: p. 476-478.

18. Lovell ME, \& Evans JH. A comparison of the spinal board and the vacuum stretcher, spinal stibility and interface pressure. Injury. 1994. 25: p. 179-180.

19. Schouten R, Albert $T$ \& Kwon BK. The spine-injured patient: initial assessment and emergency treatment. J Am Acad Orthop Surg. 2012. 20: p. 336-346.

20. Kwan I, Bunn F \& Roberts IG. Spinal immobilisation for trauma patients (review). Cochrane database of systematic reviews; 2009.

21. Hauswald M. A re-conceptualisation of acute spinal care. Emerg Med J. 2013. 30: p. 720-723.

22. White CC, D.R., Millin MG and the Standards and Clinical Practice Committee, National Association of EMS Physicians. EMS spinal precautions and the use of the long backboard - resource document to the position statement of the national association of EMS physicians and the American College of Surgeons committee on trauma. Prehosp Emerg Care. 2014. 18: p. 306-314. 
23. Berg G, Nyberg S, Harrison P, Baumchen J, Gurss E \& Hennes E. Near-infrared spectroscopy measurement of sacral tissue oxygen saturation in healthy volunteers immobilized on rigid spine boards. Prehosp Emerg Care. 2010. 14: p. 419-424.

24. DeFloor T. The risk of pressure sores: a conceptual scheme. J Clin Nurs. 1999. 8: p. 206-216.

25. Mak AFT, Yu Y, Kwan LPC, Sun L \& Tam EWC. Deformation and reperfusion damages and their accumulation in subcutaneous tissues during loading and unloading: a theoretical modeling of deep tissue injuries. J Theoretical Biology. 2011. 11: p. 65-73.

26. Gunes UY. A descriptive study of pressure ulcer pain. Ostomy Wound Manag. 2008. 54: p. 56-61.

27. Pieper B, Langemo D \& Cuddigan J. Pressure ulcer pain: a systematic literature review and National Pressure Ulcer Advisory Panel white paper. Ostomy Wound Manag. 2009. 55: p. 16-31.

28. Rastinehad D. Pressure ulcer pain. J Wound Ostomy Cont. 2006. 33: p. 252-257.

29. Fox C. Living with a pressure ulcer: a descriptive study of patients' experiences. Br J Community Nurs. 2002. 7: p. 10-22.

30. Hopkins A, Dealey C, Bale S, DeFloor T \& Worboys F. Patient stories of living with a pressure ulcer. J Adv Nurs. 2006. 56: p. 345-353.

31. Allman RM, Goode PS, Burst N, Bartolucci AA \& Thomas DR. Pressure ulcers, hospital complications, and disease severity: impact on hospital costs and length of stay. Adv Skin Wound Care. 1999. 12: p. 22-30.

32. Graves N, Birrell FA, Whitby M. Modeling the economic losses from pressure ulcers among hospitalized patients in Australia. Wound Repair Regen. 2005. 13: p. 465-467.

33. Linder-Ganz E, Engelberg S, Scheinowitz M \& Gefen A. Pressure-time cell death threshold for albino rat skeletal muscles as related to pressure sore biomechanics. J Biomech. 2006. 39: p. 2725-2732.

34. Stekelenburg A, Oomens WJ \& Bader DL. Compression-induced tissue damage: animal models, in Pressure ulcer research: current and future perspectives, Bader DL, Bouten CVC, Colin D \& Oomens CWJ (Ed). 2005, Springer-Verlag. p. 187-204.

35. Bouland AJ, Jenkins JL, \& Levy MJ. Assessing attitudes toward spinal immobilization. J Emerg Med. 2013. 45: p. e117-e125.

36. Stagg MJ, \& Lovell ME. A repeat audit of spinal board usage in the emergency department. Injury. 2008. 39: p. 323-326.

37. Black J, Baharestani MM, Cuddigan J, Dorner B, Edsberg L, Langemo D et al. National Pressure Ulcers Advisory Panel's updated pressure ulcer staging system. Adv Skin Wound Care. 2007. 20: p. 269-274.

38. European Pressure Ulcer Advisory Panel, National Pressure Ulcer Advisory Panel. Prevention and treatment of pressure ulcers: clinical practice guideline2009, Washington DC.

39. Anthony D, Papanikolaou P, Parboteeah S \& Saleh M. Do risk assessment scales for pressure ulcers work? J Tissue Viabil. 2010. 19: p. 132-136.

40. Schoonhoven L, Haalboom JRE, Bousema MT, Algra A, Grobbee DE, Grypdonck MH et al. Prospective cohort study of routine use of risk assessment scales for prediction of pressure ulcers. British Med J. 2002. 325: p. 797-801.

41. Uchi H, Terao H, Koga T \& Furue M. Cytokines and chemokines in the epidermis. J Dermatol Sci. 2000. 24: p. S29-S38.

42. Lee RT, Briggs WH, Cheng GC, Rossiter HB, Libby P \& Kupper T. Mechanical deformation promotes secretion of IL-1alpha and IL-1 receptor antagonist. J Immunol. 1997. 159: p. 5084-5088.

43. Berrington de Gonzalez A, \& Darby S. Risk of cancer from diagnostic x-rays: estimates for the UK and 14 other countries. Lancet. 2004. 363: p. 345-351.

44. Einstein AJ, Henzlova MJ \& Rajagopalan S. Estimating Risk of Cancer Associated With Radiation Exposure From 64-Slice Computed Tomography Coronary Angiography. JAMA. 2007. 298(3): p. 317323.

45. Hall EJ, \& Brenner DJ. Cancer risks from diagnostic radiology. British J Radiol. 2008. 81: p. 362-378. 



\section{Valorisation}

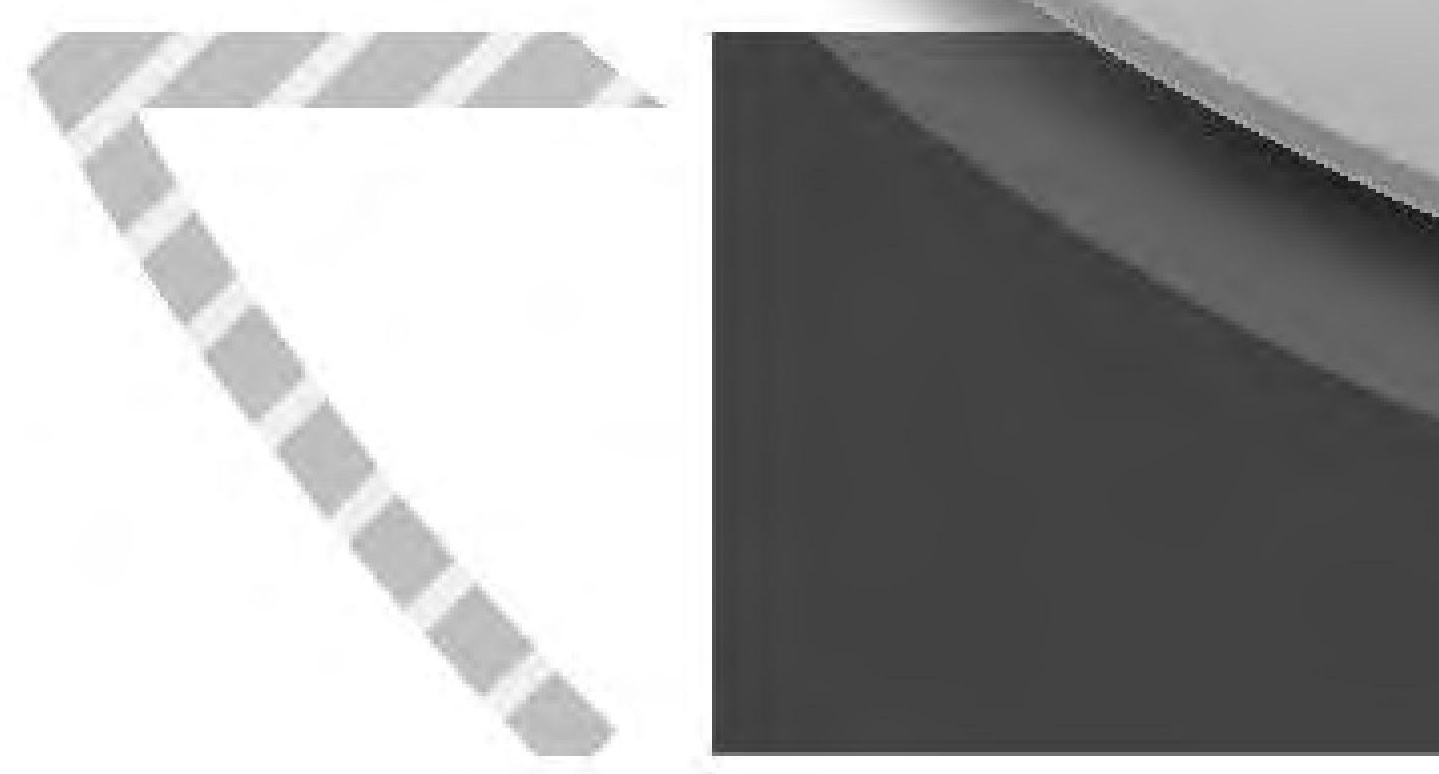

Baukje Hemmes 


\section{Valorisation}

Societal and economic relevance of the research reported in this thesis

Injuries and violence are a major public health issue worldwide and account for nearly 1 out of every 10 deaths every year, with nearly 6 million people dying each year from unintentional injury and violence. A multitude of patients are treated medically every year for physical injuries. [1]

Injuries can result in life-long disability, grossly impacting on quality of life and socio-economic welfare. Spinal cord injuries are among the most life-impacting injuries [2] and therefore need to be treated in the best possible way. Although options for definitive in-hospital care are well established [3], evidence for optimal care in the prehospital phase is often based on expert opinion [4] or even lacking completely [5]. External spinal immobilisation using a spineboard or vacuum mattress has been regarded as the gold standard of care for patients with (suspected) spinal injury for almost 80 years [6], even though no evidence is available regarding its efficacy in preventing secondary injury.

In recent years the practice of external immobilisation to prevent further damage to the spine has been heavily debated [5, 7]. Ample evidence exists on the detrimental effects of using these devices, such as pain, breathing difficulties and the risk of developing pressure ulcers, which lead to massively increased hospital stays and care costs $[8,9]$. Despite the lack of evidence about their benefits, and an ever growing body of evidence on their disadvantages, devices for external spinal immobilisation are still widely used. Important factors in keeping this practice alive are a fear of worsening injuries due to untimely treatment of the patient (although no such case has ever been documented) augmented by fear of legal repercussions [10] and the moral obligation of providing the patient with any beneficial treatment [11].

This situation necessitates research into a suitable alternative for the existing devices, providing the sought-after effect of external stabilisation and fixation, while at the same time minimising detrimental effects. We hypothesised that a soft-layered spineboard could combine these requirements and could thereby yield societal and economic benefits.

From a societal perspective, the studies reported in this thesis have proven that a soft-layered spineboard diminishes pain and the risk of developing pressure ulcers through tissue deformation and high tissue-interface pressures, while at the same time offering radiotranslucency properties similar to those of the rigid spineboard, and can therefore be recommended as best practice. In addition, our research into biological markers for soft tissue damage is important in that it adds new evidence toward the development of an objective indicator of soft-tissue damage. 
The scientific work reported in this thesis was supported by the Network Acute Care Limburg. One of the aims of the Network is to disseminate new knowledge in the domain of acute care. A critical appraisal of existing practices lies at the basis of many of the products developed by the Network. Scientific work comparing existing practices with new ones encourages innovations, facilitating implementation of these interventions. Therefore, the research results obtained by the Network may lead to the introduction of efficient interventions, but also to cessation of interventions that have proven to be inefficient.

\section{Target population}

Every year, around the world, between 250000 and 500000 people suffer a spinal cord injury ( $\mathrm{SCl}$ [12]. The vast majority of spinal cord injuries are due to incidents such as road traffic accidents, falls or violence. International protocols $[13,14]$ call for spinal immobilisation whenever spinal injury cannot be ruled out. Although the exact number of patients who undergo full spinal immobilisation is unknown, it is many times the number of those actually being diagnosed with spinal injury.

Since spineboards or vacuum mattresses are routinely applied by ambulance personnel and ER staff, results reported in this thesis apply to a large population of doctors and paramedics who work in acute care. Recommendations resulting from this thesis can be helpful in raising awareness of the detrimental effects of using a rigid spineboard or vacuum mattress, thereby encouraging care providers to make an informed decision before applying an immobilisation device. Since high-energy injuries occur worldwide and patient care for the victims involves a host of care providers, this thesis has the potential of having a global impact on practices of external spinal immobilisation.

\section{Knowledge utilisation}

The results on pain and soft tissue deformation and damage add to the body of knowledge underlying the current worldwide debate on the ongoing use of the spineboard and vacuum mattress. As such, the evidence presented in this thesis can be used in updating protocols for pre-hospital care for patients with suspected spinal injury. Furthermore, knowledge gained about the possibility to use biological markers of soft tissue damage can help guide research in the search for an objective indicator of pressure ulcers.

\section{Innovation and future perspectives}

The use of devices for external spinal immobilisation has been described in the international literature for decades. Although many studies have warned about the detrimental effects of using these devices, development of a comfortable, patient- 
friendly alternative has lagged behind. No studies other than those presented in this thesis have been published on a spineboard with integrated mattress. For this invention to be a true innovation and have an impact on society, it has to be implemented on a large scale. Since previously proposed padding on top of existing rigid spineboards has never truly made it to the workfloor, while the need for such a device is still urgent, we expect the presented soft-layered spineboard to fill a gap in the market and to be implemented in daily practice. 


\section{References}

1. World Health Orgnisation, Injuries and violence: the facts, 2010, WHO Press: Geneva, Switzerland.

2. Westgren N, \& Levi R, Quality of life and traumatic spinal cord injury. Arch Phys Med Rehabil. 1998. 79(11): p. 1433-1439.

3. Wilson R, Forgione N \& Fehlings MG, Emerging therapies for acute traumatic spinal cord injury. CMAJ, 2013. 185(6): p. 485-492.

4. Ahn H, Singh J, Nathens A et al, Pre-hospital care management of a potential spinal cord injured patient: a systematic review of the literature and evidence-based guidelines. J Neurotrauma, 2011. 28: p. 1341-1361.

5. Hauswald M, A re-conceptualisation of acute spinal care. Emerg Med J, 2013. 30: p. 720-723.

6. Warden CA, Splint stretcher board, U.S.P. Office, Editor 1938: United States of America.

7. Abram S, \& Bulstrode C, Routine spinal immobilization in trauma patients: what are the advantages and disadvantages? The Surgeon, 2010. 8: p. 218-222.

8. Kwan I, \& Bunn F, Effects of prehospital spinal immobilization: a systematic review of randomized trials on healthy subjects. Prehosp Disaster Med, 2005. 20: p. 47-53.

9. Allman RM, D.A.S.M., Pressure ulcers, hospital complications, and disease severity: impact on hospital costs and length of stay. Adv Skin Wound Care, 1999. 12: p. 22-30.

10. Butman A, Schelble DT \& Vomacka RW, The relevance of the occult cervical spine controversy and mechanism of injury to prehospital protocols: a review of the issues and literature. Prehosp Disaster Med, 1996. 11: p. 228-233.

11. Donovan WH, Ethics, health care and spinal cord injury: research, practice and finance. Spinal Cord, 2011. 49: p. 162-174.

12. World Health Orgnisation. Spinal cord injury. Factsheet No 384. 2013; Available from: http://www.who.int/mediacentre/factsheets/fs384/en/.

13. National Association of Emergency Medical Technicians, ed. PHTLS: Prehospital Trauma Life Support. 8th ed. 2014, Jones \& Bartlett Learning.

14. American College of Surgeons, ed. Advanced Trauma Life Support. Student course manual. 2012: Chicago, IL. 



\section{Dankwoord}

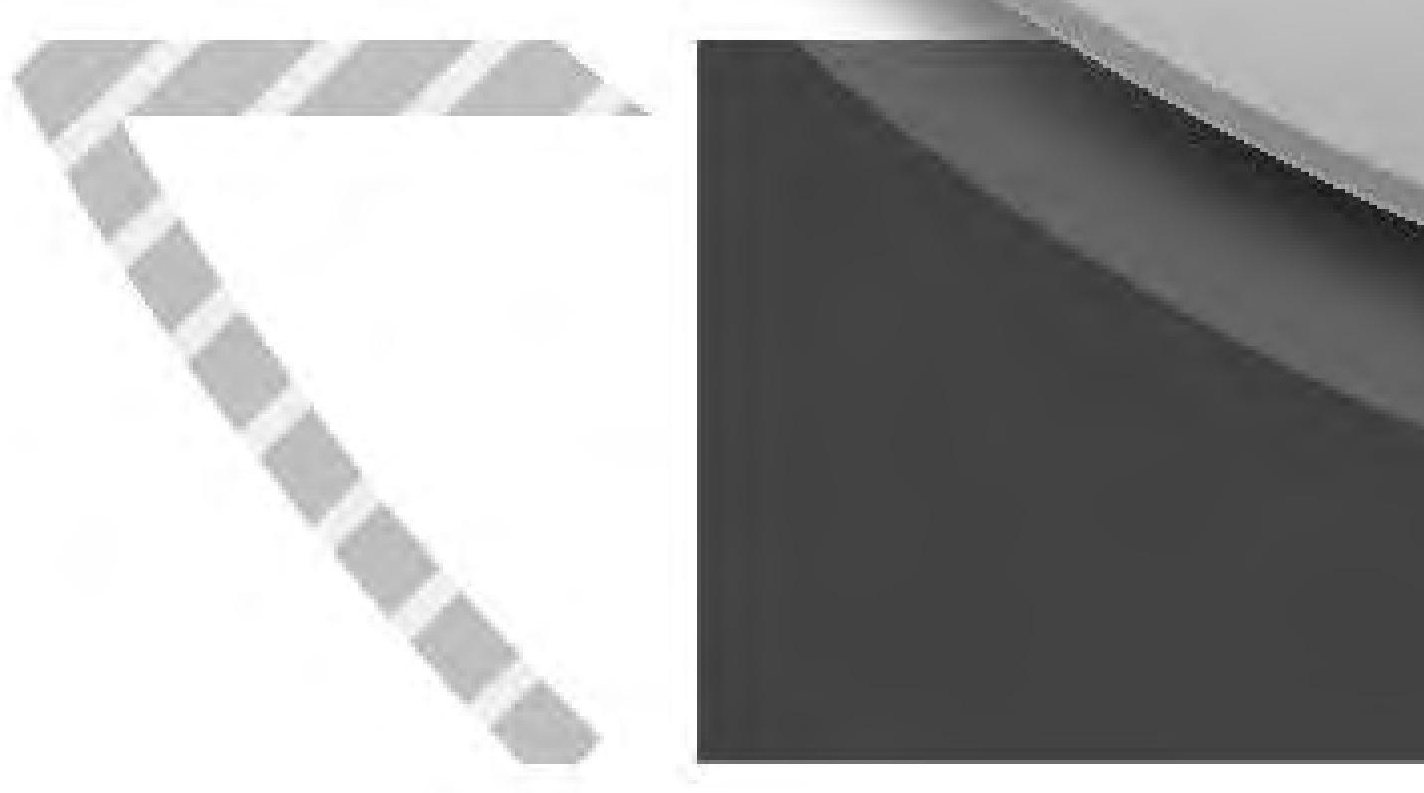




\section{Dankwoord}

Een promotietraject doorlopen doe je niet in je eentje. Ook al staat er maar één naam op de kaft van dit boekje, zonder de hulp van de mensen om mij heen had ik het niet gekund.

Professor Brink, beste Peter. Ik kan het niet vaak genoeg zeggen: dank je wel. Dank je wel voor jouw vertrouwen in mij, voor alle duwtjes in de goede richting en het geduldig uitleggen van technische details tot zelfs ik als niet-bèta het begrijp. Onze samenwerking heeft alle vormen gekend van "ik heb nog nooit een promovendus zo intensief begeleid" tot "ik hoor nooit meer wat van je"; wat fijn dat je me de ruimte hebt gegeven om het op mijn manier te doen. Dank je wel voor alle steun in de afgelopen jaren.

Dr. Poeze, beste Martijn. Wat was het een intensieve periode. Blijdschap, frustratie, overwinningen, ongeduld - je hebt het allemaal over je heen gestort gekregen. Oeverloos hebben we met de data gestoeid, tot er uiteindelijk toch altijd weer een mooie stukje statistiek uit kwam. De flauwe humor die helemaal niks met het boekje te maken had, was een mooie afwisseling voor het harde werk. Zonder jouw steun en geduld had ik de eindstreep niet gehaald.

Dr. Oomens, beste Cees. Het was voor mij een bijzondere ervaring om met een "softe" achtergrond bij de "harde" wereld van de Technische Universiteit Eindhoven terecht te komen. Ontzettend leuk om bezig te zijn met onderzoek op de scheidslijn van techniek en praktijk. Nieuwe werelden zijn voor mij open gegaan; wie had ooit gedacht dat ik iets over finite elements of cytokines op papier zou zetten. Dank je wel voor de inspirerende samenwerking.

Dr. Jeukens, beste Cécile. Jou heb ik aan het einde van het traject leren kennen als vraagbaak voor alles wat radiologie is. Het was behoorlijk stoeien met methoden en formules, maar het is toch maar mooi gelukt en ik heb zelfs het idee dat ik een heel klein beetje begrijp waar het over gaat. Dank je wel dat je die tijd en moeite in mij hebt willen investeren.

Professor Bader, dear Dan. It was a privilage to work with you. Discussing the methods and results of the cytokine study has been a great experience, always leaving me wondering whether I had done the right thing. Thank you for introducing me to the works of the EPUAP and standing behind me (literally and figuratively) during the 2014 Southampton meeting. It gave me the feeling that I was on the right track after all.

Hierbij wil ik ook de andere co-auteurs van mijn artikelen bedanken voor hun bijdragen: Willem Zenhorst, Merel Broek, Luuk de Wert, Alia Al-Haidari, Paul Hofman, Ed van der Linden, Gerrit Kemerink. Verder gaat er ook een speciale dank uit naar 
Monique Brink, die in een vroeg stadium mij de beginselen van de radiologie heeft uitgelegd. Ook alle personeel van de OK, radiologie en Spoedeisende Hulp van het MUMC wil ik bedanken voor hun niet aflatende bereidheid om mij "met die surfplank" mijn onderzoek te laten uitvoeren.

Er is nog een groep mensen die onmisbaar is geweest bij het doen van dit onderzoek: de deelnemers. Voor de onderzoeken met gezonde vrijwilligers hebben vrienden, familie en collega's zich beschikbaar gesteld. En dat hebben ze geweten. De vele collega's hebben nu -vaak voor het eerst- zelf kunnen ervaren hoe het is om op een wervelplank te liggen. Wat heerlijk om daarna op de Spoedeisende Hulp te komen en te horen hoe de verhalen zich verspreiden en te merken dat er veel meer aandacht is gekomen voor het snel verwijderen van de wervelplank. Een andere belangrijke groep deelnemers zijn de patiënten geweest, die bereid waren om tijdens hun operatie op een wervelplank te liggen, en achteraf foto's te laten maken van hun billen. Wat bijzonder dat zij dit wilden doen.

Ik wil ook mijn waardering uitspreken voor de professionals op de Spoedeisende Hulp van het MUMC die het idee van een zachte wervelplank enthousiast omarmd hebben. Het "omplanken" van de patiënt van de harde naar de zachte wervelplank is inmiddels ingeburgerd in de acute zorg voor de traumapatiënt, en daar ben ik trots op.

Ook mijn collega's van het Netwerk Acute Zorg Limburg kunnen niet ontbreken in dit rijtje. Jarenlang hebben zij mij zien rondsjouwen met planken, matrassen, headblocks en toebehoren, en regelmatig hebben ze zich al dan niet geheel vrijwillig opgeofferd om weer eens te komen liggen voor de wetenschap. Wat fijn om altijd een paar ad hoc vrijwilligers bij de hand te hebben!

Lieve collega's bij het Integraal Kankercentrum Nederland, dank voor jullie warme belangstelling - wat een fijne omgeving om dit traject af te ronden.

Naast alle dagen (en avonden en soms zelfs nachten) van werken, was er gelukkig ook tijd om af te schakelen. Het is soms best lastig om een goede balans te vinden tussen werk en privé, zeker als je in de afrondende fase zit en elke uitnodiging om iets leuks te gaan doen moet afslaan met een "nee, ik moet nog iets doen aan mijn boekje...". Borjan, Hesther, Tom, Esmee, Bas, Laura, Jeroen, Pascale, Maja, Juno, Michel, Lotje, Sebas, Marieke, Roger, Kim, Tessa, Jan-Willem, Juanita en Sjors - "ja” is voortaan weer een optie. 
Suzanne en Agnes, mijn paranimfen. Voor jullie een bijzonder dank je wel. Tijdens de verdediging van mijn proefschrift staan jullie letterlijk en figuurlijk achter mij, en ik zou het niet anders willen. Lieve Suzanne, jij was een steun en toeverlaat toen ik het het hardst nodig had. Samen hebben we heel wat hoogte- en dieptepunten doorleefd. Dat jij hier nu bij mij bent, betekent heel veel voor me. Lieve Agnes, we zijn zo'n beetje samen opgegroeid en onze bijzondere band is sterker dan ooit. De reünie op "de dijk" heeft voor mij bevestigd: jou wil ik op deze bijzondere dag in een bijzondere rol bij me hebben.

Tot slot een speciaal liefdevol dank voor mijn ouders en mijn zusje. Papa en mama, jullie hebben me altijd gesteund, in alles, in goede en in slechte tijden en dat vind ik heel bijzonder. Te weten dat deze mijlpaal voor jullie net zo'n hoogtepunt is als voor mij, maakt de dag nog mooier. Monique, zusje, wat mooi dat onze zussen-connectie alles heeft overleefd - afstand, tijd en karakters. Floris, grote kleine man, jij mag natuurlijk ook niet ontbreken. Ik hou van jullie.

En dan nu: feest! Het leven begint tenslotte bij 40 ! 
List of publications and curriculum vitae

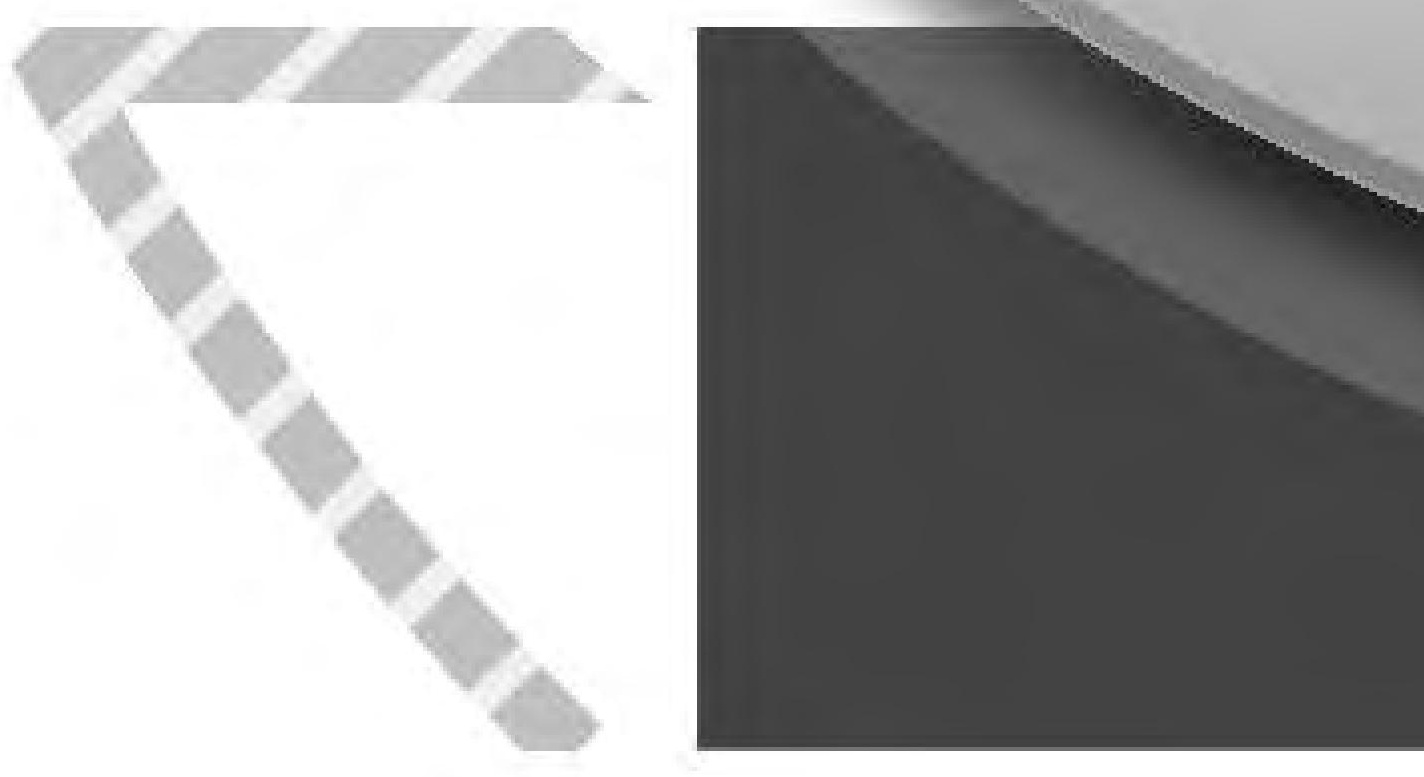




\section{List of publications}

\section{Scientific papers}

Hemmes B, Jeukens CRLPN, Kemerink GJ, Brink PRG \& Poeze M. Effect of spinal immobilization devices on radiation exposure in conventional radiography and computed tomography. Submitted.

Hemmes B, Jeukens CRLPN, Al-Haidari A, Hofman PAM, van der Linder ES, Brink PRG \& Poeze M. Effects of spineboard and headblocks on the image quality of head CT scans. Submitted.

Kalmet PHS, Koc B, Hemmes B, ten Broeke, Dekkers G, Huxtinx P et al. Effectiveness of a multidisciplinary clinical pathway for elderly patients with hip fracture: a multicenter comparative cohort study. Submitted.

Hemmes B, Poeze $M$ \& Brink PRG. Effects of unconsciousness during spinal immobilisation on tissue-interface pressures: A randomised controlled trial comparing a standard rigid spineboard with a newly developed soft-layered long spineboard. Injury, 2014. 45:1741-1746.

Oomens CWJ, Zenhorst W, Broek M, Hemmes B, Poeze M, Brink PRG \& Bader DL. A numerical study to analyse the risk for pressure ulcer development on a spine board. Clin Biomech, 2013. 28:736-742.

Aubuchon MMF, Hemmes B, Poeze M, Jansen J \& Brink PRG. Prehospital care in patients with severe traumatic brain injury: Does the level of prehospital care influence mortality? Eur J Trauma Emerg Surg, 2013. 39:35-41.

Aziz A, Hemmes B, Khalid S, Janzing HMJ, Meesters B \& Brink PRG. The less invasive stabilisation system (LISS) plate in the treatment of distal femoral fractures: does the severity of fracture make a difference to outcome? J Bone Joint Surg Br, 2012. 94-B: supp XVIII 46.

Hemmes B, Poeze M \& Brink PRG. Reduced tissue-interface pressure and increased comfort on a newly developed soft-layered long spineboard. J Trauma, 2010. 68:593598. 
Hemmes, B \& Blankendaal, F. Risicoproducten bij senioren. Tijdschrift voor gezondheidswetenschappen, 2001.78:25.

Wiers, R. W., Velde, C. van de \& Hemmes, B. (1999). Knowledge structure and type of explanation in the domain of bodily functioning. In: M.Hahn \& S.C. Stoness (Eds.) Proceedings of the 21st annual conference of the cognitive science society. Hillsdale NJ: Lawrence Erlbaum. 1999, p.826.

\section{Other contributions}

2014: Traumadagen (Amsterdam): Een retrospectief multi-center onderzoek naar 30dagen mortaliteit bij ouderen met een heupfractuur. Poster presentation.

2014: 2nd focus meeting of the European Pressure Ulcer Advisory Panel (Southampton, UK): Reduced soft tissue trauma using a new spineboard. Invited speaker.

2012: 15th Annual European Pressure Ulcer Meeting of the European Pressure Ulcer Advisory Panel (Cardiff, UK). A new spinal board to reduce soft tissue trauma during prolonged support. Invited speaker.

2009: Traumadagen (Amsterdam): Moet een MMT worden ingezet bij patiënten met een bedreigde ademweg? Poster presentation.

2009: 10th European congress of trauma and emergency surgery (Antalya, Turkije): Reduced tissue-interface pressure and increased comfort on a newly developed softlayered long spineboard. Oral presentation.

2009: 10th European congress of trauma and emergency surgery (Antalya, Turkije): Prehospital care in patients with severe traumatic brain injury. Poster presentation.

2008: Traumadagen (Utrecht): Minder kans op drukplekken en beter comfort op een nieuw ontwikkelde wervelplank. Oral presentation. 


\section{Curriculum Vitae}

Baukje Hemmes werd geboren op 21 januari 1976 te Hilversum en groeide op in Kortenhoef. Na het behalen van het middelbare school diploma aan het A. Roland Holst College in Hilversum verhuisde ze in 1994 naar Maastricht. Daar studeerde ze eerst een jaar Cultuurwetenschappen om vervolgens over te stappen naar Psychologie, waar ze veel aandacht aan commissiewerk besteedde. Na haar studie heeft Baukje drie jaar in Amsterdam gewerkt voor Veiligheid.nl, een stichting die zich richt op ongevallenpreventie. Daarna keerde ze terug naar Maastricht waar ze de masteropleiding Health Education and Promotion volgde. Tijdens die studie kwam ze te werken bij het Traumacentrum Limburg, tegenwoordig Netwerk Acute Zorg Limburg. Hier heeft Baukje een grote belangstelling voor de ambulancerzorg ontwikkeld. Dit ging samen met haar ontwikkeling als onderzoeker, en heeft geresulteerd in het proefschrift dat nu voor $u$ ligt. Op dit moment werkt Baukje bij het Intergraal Kankercentrum Nederland en is ze vrijwilliger voor de opvang van zwerfkittens bij de Dierenbescherming. 


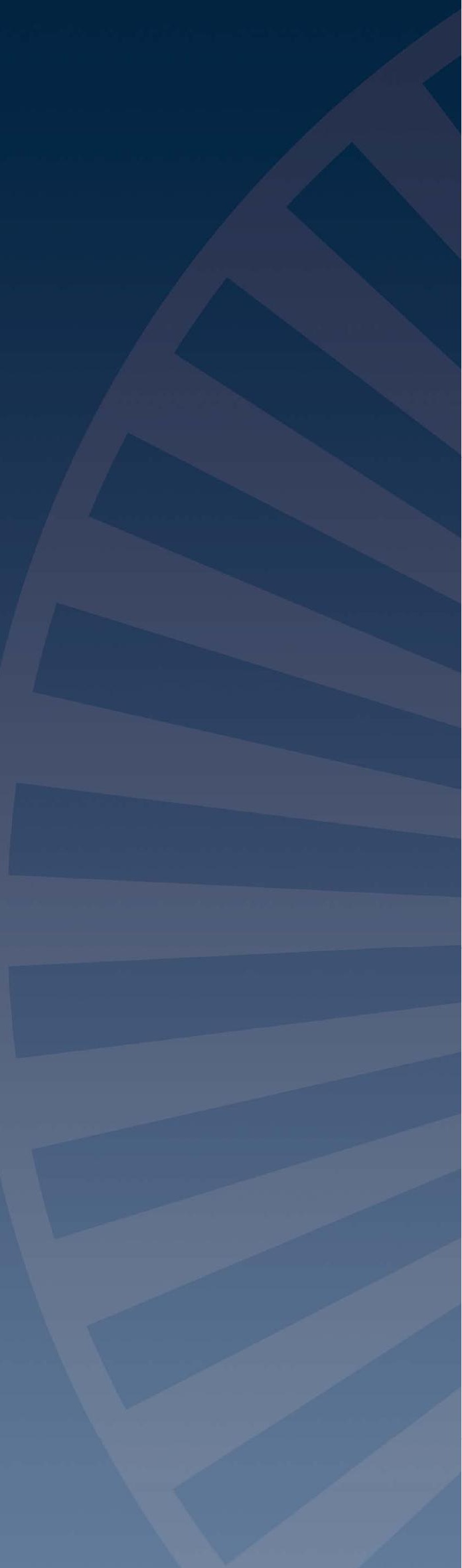

